\title{
CLASH: MASS DISTRIBUTION IN AND AROUND MACS J1206.2-0847 FROM A FULL CLUSTER LENSING ANALYSIS*
}

\author{
Keitchi Umetsu ${ }^{1}$, Elinor Medezinski ${ }^{2}$, Mario Nonino $^{3}$, Julian Merten ${ }^{4}$, Adi Zitrin ${ }^{5}$, Alberto Molino $^{6}$, \\ Claudio Grillo $^{7}$, Mauricio Carrasco ${ }^{8,9}$, Megan Donahue $^{10}$, Andisheh Mahdavi $^{11}$, Dan CoE $^{12}$, Marc Postman $^{12}$, \\ Anton Koekemoer ${ }^{12}$, Nicole Czakon ${ }^{13}$, Jack Sayers $^{13}$, Tony Mroczkowski ${ }^{4,13,28}{ }^{\text {, Sunil Golwala }}{ }^{13}$, Patrick M. Koch ${ }^{1}$, \\ Kai-Yang Lin ${ }^{1}$, Sandor M. Molnar ${ }^{14}$, Piero Rosati ${ }^{9}$, Italo Balestra ${ }^{3}$, Amata Mercurio ${ }^{15}$, Marco Scodeggio ${ }^{16}$, \\ Andrea Biviano $^{3}$, Timo Anguita ${ }^{8,17}$, Leopoldo Infante ${ }^{8}$, Gregor Seidel $^{17}$, Irene Sendra ${ }^{18}$, Stephanie Jouvel ${ }^{19,20}$, \\ Ole Host $^{19}$, Doron Lemze ${ }^{2}$, Tom Broadhurst ${ }^{18,21}$, Massimo Meneghetti ${ }^{22}$, Leonidas MoustaKas ${ }^{4}$, \\ Matthias BartelmanN ${ }^{5}$, Narciso Benítez ${ }^{6}$, Rychard Bouwens $^{23}$, LARry Bradley ${ }^{12}$, Holland Ford ${ }^{2}$, \\ Yolanda Jiménez-Teja ${ }^{6}$, Daniel Kelson ${ }^{24}$, Ofer Lahav ${ }^{19}$, Peter Melchior ${ }^{25}$, John Moustakas ${ }^{26}$, \\ Sara Ogaz ${ }^{12}$, Stella Seitz $^{27}$, AND Wei ZhenG ${ }^{2}$ \\ ${ }^{1}$ Institute of Astronomy and Astrophysics, Academia Sinica, P.O. Box 23-141, Taipei 10617, Taiwan; keiichi@asiaa.sinica.edu.tw \\ ${ }^{2}$ Department of Physics and Astronomy, The Johns Hopkins University, 3400 North Charles Street, Baltimore, MD 21218, USA \\ ${ }^{3}$ INAF/Osservatorio Astronomico di Trieste, via G. B. Tiepolo 11, 34143 Trieste, Italy \\ ${ }^{4}$ Jet Propulsion Laboratory, California Institute of Technology, MS 169-327, Pasadena, CA 91109, USA \\ ${ }^{5}$ Institut für Theoretische Astrophysik, ZAH, Heidelberg, Germany \\ ${ }^{6}$ Instituto de Astrofísica de Andalucía (CSIC), Granada, Spain \\ ${ }^{7}$ Dark Cosmology Centre, Niels Bohr Institute, University of Copenhagen, Juliane Mariesvej 30, DK-2100 Copenhagen, Denmark \\ ${ }^{8}$ Centro de Astro-Ingeniería, Departamento de Astronomía y Astrofísica, Pontificia Universidad Católica de Chile, V. Mackenna 4860, Santiago, Chile \\ ${ }^{9}$ ESO-European Southern Observatory, D-85748 Garching bei München, Germany \\ ${ }^{10}$ Department of Physics and Astronomy, Michigan State University, East Lansing, MI 48824, USA \\ ${ }^{11}$ Department of Physics and Astronomy, San Francisco State University, Thornton Hall 527, CA, USA \\ ${ }^{12}$ Space Telescope Science Institute, 3700 San Martin Drive, Baltimore, MD 21208, USA \\ ${ }^{13}$ Division of Physics, Math, and Astronomy, California Institute of Technology, Pasadena, CA 91125, USA \\ ${ }^{14}$ LeCosPA Center, National Taiwan University, Taipei 10617, Taiwan \\ ${ }^{15}$ INAF-Osservatorio Astronomico di Capodimonte, via Moiariello 16, I-80131 Napoli, Italy \\ ${ }^{16}$ INAF-IASF Milano, via Bassini 15, I-20133 Milano, Italy \\ ${ }^{17}$ Max-Planck-Institut für Astronomie, Königstuhl 17, D-69117 Heidelberg, Germany \\ ${ }^{18}$ Department of Theoretical Physics and History of Science, University of the Basque Country UPV/EHU, P.O. Box 644, 48080 Bilbao, Spain \\ ${ }^{19}$ Department of Physics and Astronomy, University College London, London, UK \\ ${ }^{20}$ Institut de Ciéncies de l'Espai (IEEC-CSIC), E-08193 Bellaterra (Barcelona), Spain \\ ${ }^{21}$ Ikerbasque, Basque Foundation for Science, Alameda Urquijo, 36-5 Plaza Bizkaia 48011, Bilbao, Spain \\ 22 INAF, Osservatorio Astronomico di Bologna; INFN, Sezione di Bologna, Bologna, Italy \\ ${ }^{23}$ Leiden Observatory, Leiden University, Leiden, The Netherlands \\ ${ }^{24}$ Observatories of the Carnegie Institution of Washington, Pasadena, CA, USA \\ ${ }^{25}$ Center for Cosmology and Astro-Particle Physics \& Department of Physics, The Ohio State University, Columbus, OH, USA \\ ${ }^{26}$ Center for Astrophysics and Space Sciences, University of California at San Diego, 9500 Gilman Dr., MC 0424, La Jolla, CA 92093, USA \\ ${ }^{27}$ Universitäts-Sternwarte, München, Scheinerstr. 1, D-81679 München, Germany \\ Received 2012 April 4; accepted 2012 June 5; published 2012 July 26
}

\begin{abstract}
We derive an accurate mass distribution of the galaxy cluster MACS J1206.2-0847 $(z=0.439)$ from a combined weak-lensing distortion, magnification, and strong-lensing analysis of wide-field Subaru $B V R_{\mathrm{c}} I_{\mathrm{c}} z^{\prime}$ imaging and our recent 16-band Hubble Space Telescope observations taken as part of the Cluster Lensing And Supernova survey with Hubble program. We find good agreement in the regions of overlap between several weak- and strong-lensing mass reconstructions using a wide variety of modeling methods, ensuring consistency. The Subaru data reveal the presence of a surrounding large-scale structure with the major axis running approximately northwest-southeast (NW-SE), aligned with the cluster and its brightest galaxy shapes, showing elongation with a $\sim 2: 1$ axis ratio in the plane of the sky. Our full-lensing mass profile exhibits a shallow profile slope $d \ln \Sigma / d \ln R \sim-1$ at cluster outskirts $\left(R \gtrsim 1 \mathrm{Mpc} h^{-1}\right)$, whereas the mass distribution excluding the NW-SE excess regions steepens farther out, well described by the Navarro-Frenk-White form. Assuming a spherical halo, we obtain a virial mass $M_{\text {vir }}=(1.1 \pm 0.2 \pm 0.1) \times 10^{15} M_{\odot} h^{-1}$ and a halo concentration $c_{\text {vir }}=6.9 \pm 1.0 \pm 1.2\left(c_{\text {vir }} \sim 5.7\right.$ when the central $50 \mathrm{kpc} h^{-1}$ is excluded), which falls in the range $4 \lesssim\langle c\rangle \lesssim 7$ of average $c(M, z)$ predictions for relaxed clusters from recent $\Lambda$ cold dark matter simulations. Our full-lensing results are found to be in agreement with $\mathrm{X}$-ray mass measurements where the data overlap, and when combined with Chandra gas mass measurements, they yield a cumulative gas mass fraction of $13.7_{-3.0}^{+4.5} \%$ at $0.7 \mathrm{Mpc} h^{-1}\left(\approx 1.7 r_{2500}\right)$, a typical value observed for high-mass clusters.
\end{abstract}

Key words: cosmology: observations - dark matter - galaxies: clusters: individual (MACS J1206.2-0847) gravitational lensing: strong - gravitational lensing: weak

Online-only material: color figures 


\section{INTRODUCTION}

Clusters of galaxies are the largest self-gravitating systems in the universe. These massive clusters contain rich astrophysical and cosmological information about the initial conditions for cosmic structure formation and assembly of structure over cosmic time. Statistical and detailed individual properties of clusters can therefore provide fundamental constraints on models of cosmic structure formation (e.g., Allen et al. 2004; Vikhlinin et al. 2009), the unknown nature of dark matter (DM; Markevitch et al. 2004; Clowe et al. 2006), and possible modifications of the law of gravity (Narikawa \& Yamamoto 2012), complementing cosmic microwave background, galaxy clustering, and Type Ia supernova observations (Komatsu et al. 2011; Percival et al. 2010; Riess et al. 1998).

Observations of clusters have provided independent pieces of empirical evidence for the existence of DM (e.g., Zwicky 1959; Markevitch et al. 2004; Clowe et al. 2006; Okabe \& Umetsu 2008; Mahdavi et al. 2007a). A prime example of this comes from combined X-ray and lensing observations of the "Bullet system," which is understood to be the result of a high-speed collision of two cluster components occurring approximately in the plane of the sky, displaying a prominent bow shock proceeding the cool, bullet-like gas subcluster, lying between the two distinct clusters (Markevitch et al. 2004). For this system, the bulk of mass is shown to be associated with the bimodal distribution of cluster member galaxies, supporting that DM is effectively collisionless as galaxies on sub-Mpc scales (Clowe et al. 2006). Such displacements between the gas and mass distributions are quite common in merging systems and exhibit a complex variety of merging configurations (Okabe \& Umetsu 2008; Mahdavi et al. 2007a; Merten et al. 2011).

Substantial progress has been made through numerical simulation in understanding the formation and structure of collisionless DM halos in quasi equilibrium, governed by nonlinear gravitational growth of cosmic density perturbations. In the standard $\Lambda$ cold dark matter $(\Lambda \mathrm{CDM})$ paradigm of hierarchical structure formation, cluster-sized DM halos form through successive mergers of smaller halos, as well as through smooth accretion of matter along surrounding filamentary structures (Colberg et al. 2000; Shaw et al. 2006; Gao et al. 2012). In this context, the hierarchical buildup of clusters proceeds in a highly anisotropic configuration where infall and merging of matter tend to occur along preferential directions (Colberg et al. 2005), leading to the emergence of the filamentary network of matter, as observed in large galaxy redshift surveys (e.g., Colless et al. 2001; Tegmark et al. 2004; Geller et al. 2011). Cluster halos are located at dense nodes where the filaments intersect, generally triaxial reflecting the collisionless nature of DM, and elongated in the preferential infall direction of subhalos, namely, along surrounding filaments (Shaw et al. 2006).

The internal structure of DM halos constitutes one of the most distinct predictions for the CDM paradigm. $N$-body simulations of collisionless CDM established a nearly self-similar form for the spherically averaged density profile $\langle\rho(r)\rangle$ of DM halos (Navarro et al. 1997, hereafter Navarro-Frenk-White, NFW) over a wide range of halo masses, with some intrinsic variance associated with the mass assembly histories of individual halos

\footnotetext{
* Based in part on data collected at the Subaru Telescope, which is operated by the National Astronomical Society of Japan.

28 NASA Einstein Postdoctoral Fellow.
}

(Jing \& Suto 2000; Tasitsiomi et al. 2004; Graham et al. 2006; Navarro et al. 2010; Gao et al. 2012). The logarithmic gradient $\gamma_{3 \mathrm{D}}(r)=-d \ln \rho / d \ln r$ of the NFW form flattens progressively toward the center, with an inner slope flatter than a purely isothermal structure $\left(\gamma_{3 \mathrm{D}}=2\right)$ interior to the inner characteristic radius $r_{s}$ providing a distinctive, fundamental prediction for the empirical form of CDM halos. A useful index of the degree of concentration is $c_{\mathrm{vir}}=r_{\mathrm{vir}} / r_{s}$, which compares the virial radius $r_{\mathrm{vir}}$ to $r_{s}$. Halo concentration is predicted to correlate with halo mass since DM halos that are more massive collapse later when the mean background density of the universe is correspondingly lower (Bullock et al. 2001; Zhao et al. 2003; Neto et al. 2007). This prediction for the halo $c_{\mathrm{vir}}-M_{\mathrm{vir}}$ relation and its evolution has been examined by several independent large-scale simulations (e.g., Navarro et al. 1997; Bullock et al. 2001; Neto et al. 2007; Duffy et al. 2008; Klypin et al. 2011; Bhattacharya et al. 2011), with sufficient detail to establish the inherent scatter of this relation around the mean, arising from variations in the formation epoch of individual halos of given mass (Wechsler et al. 2002; Neto et al. 2007; Zhao et al. 2009).

Galaxy clusters act as powerful gravitational lenses (e.g., Bartelmann \& Schneider 2001; Umetsu 2010; Kneib \& Natarajan 2011), providing a direct probe for testing these welldefined predictions of halo structure because they are expected to have a relatively shallow mass profile with a pronounced radial curvature. A detailed examination of the $\Lambda \mathrm{CDM}$ predictions by cluster lensing has been the focus of our preceding work (Broadhurst et al. 2005a, 2008; Umetsu et al. 2007, 2009, 2010, 2011a, 2011b; Umetsu \& Broadhurst 2008).

Recent detailed lensing analyses have shown that the projected cluster mass profiles constructed from combined weakand strong-lensing data have a gradually steepening logarithmic gradient, in agreement with the predicted form for the family of collisionless CDM halos in virial equilibrium (Gavazzi et al. 2003; Broadhurst et al. 2005a, 2008; Limousin et al. 2007; Umetsu \& Broadhurst 2008; Newman et al. 2009; Umetsu et al. 2010, 2011a, 2011b; Zitrin et al. 2010, 2011c; Oguri et al. 2012; Coe et al. 2012). Intriguingly, however, some of these results reveal a relatively high degree of mass concentration in high-mass lensing clusters (e.g., Gavazzi et al. 2003; Kneib et al. 2003; Broadhurst et al. 2008; Oguri et al. 2009; Zitrin et al. 2011c), lying well above the $c_{\mathrm{vir}}-M_{\text {vir }}$ relation for clustersized halos $\left(c_{\mathrm{vir}} \sim 4-5\right.$ for CDM halos with $M_{\mathrm{vir}} \gtrsim 10^{15} M_{\odot}$ in the local universe) predicted by the $\Lambda$ CDM model, despite careful attempts to correct for sizable $(\sim 50 \%-100 \%)$ projection and selection biases inherent to lensing by triaxial halos (Hennawi et al. 2007; Meneghetti et al. 2010b, 2011). The effects of baryons on the total mass profile are generally found to only modify cluster concentrations at the $\sim 10 \%$ level (Mead et al. 2010; Duffy et al. 2010), although some studies suggest that low-mass systems $\left(M_{\mathrm{vir}} \lesssim 5 \times 10^{14} M_{\odot}\right)$ may be significantly affected by the effects of baryonic cooling (Fedeli 2012; Oguri et al. 2012). This apparent overconcentration of lensing clusters is also indicated by the generally large Einstein radii determined from strong-lensing observations (Broadhurst \& Barkana 2008; Meneghetti et al. 2010a; Zitrin et al. 2011a). These lensing results could suggest either substantial additional mass projected along the line of sight, due partly to halo triaxiality (Oguri et al. 2005), or an intrinsically higher-than-predicted concentration of mass; the latter could imply that clusters formed earlier than predicted by $\mathrm{N}$-body simulations of the current concordance $\Lambda \mathrm{CDM}$ cosmology. 
Table 1

Properties of the Galaxy Cluster MACS1206

\begin{tabular}{|c|c|}
\hline Parameter & Value \\
\hline 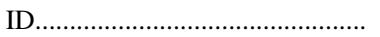 & MACS J1206.2-0847 \\
\hline \multicolumn{2}{|l|}{ Optical center position ( $\mathrm{J} 2000.0)$} \\
\hline 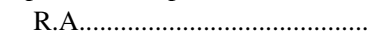 & $12: 06: 12.15$ \\
\hline 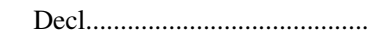 & $-08: 48: 03.4$ \\
\hline \multicolumn{2}{|l|}{ X-ray peak position $(\mathrm{J} 2000.0)$} \\
\hline R.A & $12: 06: 12.28$ \\
\hline 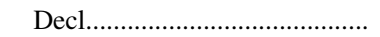 & $-08: 48: 02.4$ \\
\hline Redshift................................... & 0.4385 \\
\hline $\mathrm{X}$-ray temperature $(\mathrm{keV}) \ldots \ldots \ldots \ldots \ldots$ & $10.9 \pm 0.6$ \\
\hline Einstein radius $\left({ }^{\prime \prime}\right) \ldots \ldots \ldots \ldots \ldots \ldots \ldots \ldots$ & $28 \pm 3(17 \pm 2)$ at $z_{s}=2.54(1.03)$ \\
\hline
\end{tabular}

Notes. The cluster MACS J1206.2-0847 $(z=0.4385)$ was discovered in the Massive Cluster Survey (MACS) as described by Reference (1). The optical cluster center is defined as the center of the BCG from Reference (2). Units of right ascension are hours, minutes, and seconds, and units of declination are degrees, arcminutes, and arcseconds. The X-ray properties are taken from Reference (3). See also Reference (1). The BCG is located within $\approx 2^{\prime \prime}$ (a projected separation of $\approx 9 \mathrm{kpc} h^{-1}$ ) of the X-ray emission peak. The Einstein radii are constrained by detailed strong-lens modeling by Reference (2).

References. (1) Ebeling et al. 2009; (2) Zitrin et al. 2012; (3) Postman et al. 2012.

The Cluster Lensing And Supernova survey with Hubble (CLASH; Postman et al. 2012) ${ }^{29}$ has been in progress to obtain accurate cluster mass profiles for a sizable sample of representative clusters by combining high-quality strong- and weaklensing measurements, in combination with the complementary Subaru wide-field imaging (e.g., Umetsu et al. 2011a, 2011b). CLASH is a 524-orbit multi-cycle treasury Hubble Space Telescope (HST) program to observe 25 clusters of galaxies at $0.18<z<0.89$, each in 16 filters with the Wide Field Camera 3 (WFC3; Kimble et al. 2008) and the Advanced Camera for Surveys (ACS; Ford et al. 2003), ranging from the UV, through the optical, to the IR. Importantly, 20 CLASH clusters were $\mathrm{X}$-ray selected to be massive and relatively relaxed. This selection avoids the strong bias toward high concentrations in previously well-studied clusters selected for their strong-lensing strength, allowing us to meaningfully examine the $c-M$ relation over a sufficiently wide mass and redshift range for a cluster sample that is largely free of lensing bias (Postman et al. 2012).

In this paper we present a comprehensive weak- and strong-lensing analysis of the X-ray-selected CLASH cluster MACS J1206.2-0847 (hereafter MACS1206; see Table 1) at $z=0.439$ based on the Subaru wide-field $B V R_{\mathrm{c}} I_{\mathrm{c}} z^{\prime}$ imaging, combined with our recent CLASH HST imaging and Very Large Telescope (VLT)/VIMOS spectroscopic observations presented in Zitrin et al. (2012), who carried out a detailed strong-lensing analysis of the cluster. MACS1206 is an X-ray luminous cluster (Ebeling et al. 2009), originally discovered in the Massive Cluster Survey (MACS; Ebeling et al. 2001, 2009). Therefore, it is an interesting target for detailed lensing analyses to compare with well-studied, lensing-selected clusters (e.g., Umetsu et al. 2011a, 2011b; Oguri et al. 2009, 2012).

The paper is organized as follows. In Section 2 we briefly summarize the basic theory of cluster weak gravitational lensing. In Section 3 we describe details of the full weak-lensing analysis of Subaru observations. In Section 4 we present results from several semi-independent strong-lensing analyses to test the consistency of our strong-lens modeling. In Section 5

\footnotetext{
29 http://www.stsci.edu/ postman/CLASH
}

we derive cluster weak-lensing profiles from Subaru data. In Section 6 we combine our weak-lensing measurements with inner strong-lensing-based information from CLASH HST observations to make a full determination of the cluster mass profiles; then, we examine the radial dependence of the cluster mass distribution based on the full-lensing analysis. In Section 7 we assess carefully various sources of potential systematic uncertainties in the cluster mass and concentration measurements and discuss our results along with our complementary X-ray and Sunyaev-Zel'dovich effect (SZE) observations. Finally, a summary is given in Section 8.

Throughout this paper, we use the $\mathrm{AB}$ magnitude system and adopt a concordance $\Lambda$ CDM cosmology with $\Omega_{m}=0.3$, $\Omega_{\Lambda}=0.7$, and $H_{0}=100 \mathrm{hm} \mathrm{s}^{-1} \mathrm{Mpc}^{-1}$ with $h=0.7$. In this cosmology, $1^{\prime}$ corresponds to $238 \mathrm{kpc} h^{-1}=341 \mathrm{kpc}$ at the cluster redshift, $z=0.439$. We use the standard notation $M_{\Delta} \equiv M_{3 \mathrm{D}}\left(<r_{\Delta}\right)$ to denote the total mass enclosed within a sphere of radius $r_{\Delta}$, within which the mean interior density is $\Delta$ times the critical mass density at the cluster redshift. We refer all our virial quantities to an overdensity $\Delta$ of $\Delta_{\text {vir }} \approx 132$ based on the spherical collapse model (Appendix A of Kitayama \& Suto 1996). ${ }^{30}$ All quoted errors are $68.3 \%$ confidence limits (CL) unless otherwise stated. The reference sky position is the center of the brightest cluster galaxy (BCG) of Zitrin et al. (2012), R.A. $=12: 06: 12.15$, decl. $=-08: 48: 03.4$ (J2000.0).

\section{BASIC THEORY OF GALAXY CLUSTER WEAK LENSING}

The central quantity of interest in this work is the convergence of gravitational lensing, $\kappa(\boldsymbol{\theta})=\Sigma(\boldsymbol{\theta}) / \Sigma_{\text {crit }}$, which is the surfacemass density projected onto the lens plane, $\Sigma(\boldsymbol{\theta})$, in units of the critical surface-mass density for lensing,

$$
\Sigma_{\text {crit }}=\frac{c^{2}}{4 \pi G D_{l}} \beta^{-1} ; \quad \beta\left(z_{s}\right) \equiv \max \left[0, \frac{D_{l s}\left(z_{s}\right)}{D_{s}\left(z_{s}\right)}\right] .
$$

Here $D_{s}, D_{l}$, and $D_{l s}$ are the proper angular-diameter distances from the observer to the source, from the observer to the lens, and from the lens to the source, respectively; $\beta$ is the angular-diameter distance ratio associated with the population of background sources.

The lens distortion and magnification of images are described by the Jacobian matrix $\mathcal{A}_{\alpha \beta}(\alpha, \beta=1,2)$ of the lens mapping, which can be decomposed as $\mathcal{A}_{\alpha \beta}=(1-\kappa) \delta_{\alpha \beta}-\Gamma_{\alpha \beta}$, where $\delta_{\alpha \beta}$ is Kronecker's delta and $\Gamma_{\alpha \beta}$ is the trace-free, symmetric shear matrix,

$$
\Gamma=\left(\begin{array}{cc}
+\gamma_{1} & \gamma_{2} \\
\gamma_{2} & -\gamma_{1}
\end{array}\right)
$$

with the components of complex gravitational shear with spin-2 nature (under coordinate rotations; see Bartelmann \& Schneider 2001; Okura et al. 2008), $\gamma=\gamma_{1}+i \gamma_{2} \equiv|\gamma| e^{2 i \phi_{\gamma}}$. The $\kappa$ and $\gamma$ fields are related to each other by

$$
\triangle \kappa(\boldsymbol{\theta})=\partial^{\alpha} \partial^{\beta} \Gamma_{\alpha \beta}(\boldsymbol{\theta}) .
$$

The Green's function for the two-dimensional (2D) Poisson equation is $\triangle^{-1}\left(\boldsymbol{\theta}, \boldsymbol{\theta}^{\prime}\right)=\ln \left|\boldsymbol{\theta}-\boldsymbol{\theta}^{\prime}\right| /(2 \pi)$, so that Equation (3) can be readily solved (Kaiser \& Squires 1993).

In the strict weak-lensing limit $(\kappa,|\gamma| \ll 1), \Gamma_{\alpha \beta}$ induces a small quadrupole distortion of the background image, which

\footnotetext{
$\overline{30} \Delta_{\mathrm{vir}} \approx 134$ using the fitting formula given by Bryan \& Norman (1998).
} 
can be measured from observable ellipticities of background galaxy images (Kaiser et al. 1995). In general, the observable quantity for quadrupole weak lensing is not $\gamma$ but the reduced gravitational shear,

$$
g \equiv g_{1}+i g_{2}=\frac{\gamma}{1-\kappa}
$$

in the subcritical regime where $\operatorname{det} \mathcal{A}>0\left(\right.$ or $1 / g^{*}$ in the negative parity region with $\operatorname{det} \mathcal{A}<0$ ).

Given an arbitrary circular loop of radius $\theta$ on the sky, the tangential shear $\gamma_{+}(\theta)$ averaged around the loop satisfies the following identity (e.g., Kaiser 1995):

$$
\gamma_{+}(\theta)=\bar{\kappa}(<\theta)-\kappa(\theta),
$$

where $\kappa(\theta)$ is the azimuthal average of $\kappa$ around the loop and $\bar{\kappa}(<\theta)$ is the average convergence within the loop. Hence, a constant mass sheet cannot be constrained using the shear information alone, known as the mass-sheet degeneracy (e.g., Bartelmann \& Schneider 2001).

This inherent degeneracy can be unambiguously broken by measuring the magnification effects, which provide complementary and independent constraints on the cluster mass distribution (Umetsu et al. 2011b). The magnification is given by the inverse Jacobian determinant,

$$
\mu(\boldsymbol{\theta})=\frac{1}{|\operatorname{det} \mathcal{A}(\boldsymbol{\theta})|}=\frac{1}{\left.\left|(1-\kappa)^{2}-\right| \gamma\right|^{2} \mid} .
$$

The magnification $\mu(\boldsymbol{\theta})$ can influence the observed surface density $n_{\mu}(\boldsymbol{\theta})$ of background sources, expanding the area of sky, and enhancing the observed flux of background sources (e.g., Broadhurst et al. 1995; Umetsu \& Broadhurst 2008; Van Waerbeke et al. 2010; Rozo \& Schmidt 2010; Umetsu et al. 2011b; Hildebrandt et al. 2011; Ford et al. 2011). The former effect reduces the effective observing area in the source plane, decreasing the number of sources per solid angle; on the other hand, the latter effect amplifies the flux of background sources, thereby increasing the number of sources above the limiting flux. The net effect is known as magnification bias and depends on the intrinsic slope of the luminosity function of background sources as

$$
n_{\mu}(\boldsymbol{\theta})=n_{0} \mu(\boldsymbol{\theta})^{2.5 s-1},
$$

where $n_{0}=d N_{0}\left(<m_{\text {cut }}\right) / d \Omega$ is the unlensed mean number density of background sources for a given magnitude cutoff $m_{\text {cut }}$, approximated locally as a power-law cut with slope $s=d \log _{10} N_{0}(<m) / d m>0$. In the strict weak-lensing limit, $n_{\mu} / n_{0}-1 \approx(5 s-2) \kappa$. For a maximally depleted population of galaxies with $s=0, n_{\mu} / n_{0}=\mu^{-1} \approx 1-2 \kappa$ in this limit.

Alternatively, the mass-sheet degree of freedom (dof) can be determined such that the mean $\Sigma$ averaged over the outermost cluster region vanishes, if a sufficiently wide sky coverage is available. $^{31}$

\section{SUBARU DATA AND ANALYSIS}

In this section we present a technical description of our weaklensing analysis of MACS1206 based on deep Subaru multicolor images. The data reduction and the photometry procedure are summarized in Section 3.1. The details of our weak-lensing

\footnotetext{
31 Or, one may constrain the constant such that the enclosed mass within a certain aperture is consistent with cluster mass estimates from some other observations (e.g., Umetsu \& Futamase 2000).
}

Table 2

Subaru Suprime-Cam Data

\begin{tabular}{lccc}
\hline \hline Filter & $\begin{array}{c}\text { Exposure Time } \\
(\mathrm{ks})\end{array}$ & $\begin{array}{c}\text { Seeing } \\
(\operatorname{arcsec})\end{array}$ & $\begin{array}{c}m_{\text {lim }} \mathrm{c}^{\mathrm{c}} \\
(\mathrm{AB} \text { mag })\end{array}$ \\
\hline$B$ & 2.4 & 1.01 & 26.5 \\
$V$ & 2.2 & 0.95 & 26.5 \\
$R_{\mathrm{c}}$ & 2.9 & 0.78 & 26.2 \\
$I_{\mathrm{c}}$ & 3.6 & 0.71 & 26.0 \\
$z^{\prime}$ & 1.6 & 0.58 & 25.0 \\
\hline
\end{tabular}

Notes.

a Total exposure time.

b Seeing FWHM in the full stack of images.

c Limiting magnitude for a $3 \sigma$ detection within a 2 " aperture.

shape analysis are given in Section 3.2. Our shear calibration strategy is described in Section 3.3. Details of the sample selection and lensing depth estimation are given in Sections 3.4 and 3.5 , respectively.

\subsection{Subaru Data and Photometry}

We analyze deep $B V R_{\mathrm{c}} I_{\mathrm{c}} z^{\prime}$ images of MACS1206 observed with the wide-field camera Suprime-Cam $\left(34^{\prime} \times 27^{\prime}\right.$; Miyazaki et al. 2002) at the prime focus of the $8.3 \mathrm{~m}$ Subaru telescope. The observations are available in the Subaru archive, SMOKA. ${ }^{32}$ The seeing FWHM in the co-added mosaic image is $1^{\prime \prime} .01$ in $B$ $(2.4 \mathrm{ks}), 0^{\prime \prime} 95$ in $V(2.2 \mathrm{ks}), 0^{\prime \prime} .78$ in $R_{\mathrm{c}}(2.9 \mathrm{ks}), 0^{\prime} .71$ in $I_{\mathrm{c}}$ $(3.6 \mathrm{ks})$, and $0^{\prime \prime} .58$ in $z^{\prime}(1.6 \mathrm{ks})$ with $0^{\prime \prime} .20 \mathrm{pixel}^{-1}$, covering a field of approximately $36^{\prime} \times 34^{\prime}$. The limiting magnitudes are obtained as $B=26.5, V=26.5, R_{\mathrm{c}}=26.2, I_{\mathrm{c}}=26.0$, and $z^{\prime}=25.0 \mathrm{mag}$ for a $3 \sigma$ limiting detection within a $2^{\prime \prime}$ diameter aperture. The observation details of MACS1206 are listed in Table 2. Figure 1 shows Subaru $B V R_{\mathrm{c}} I_{\mathrm{c}} z^{\prime}$ composite color images of the cluster field, produced automatically using the publicly available Trilogy software (Coe et al. 2012). ${ }^{33}$

Standard reduction steps were performed using the mscred task in IRAF. ${ }^{34}$ We closely follow the data reduction procedure outlined in Nonino et al. (2009) to create a co-added mosaic of Subaru Suprime-Cam images, incorporating additional reduction steps, such as automated masking of bleeding of bright saturated stars.

To obtain an accurate astrometric solution for Subaru observations, we retrieved processed MegaCam griz images from the Canada-France-Hawaii Telescope (CFHT) archive $^{35}$ and used MegaCam $r$ data (Filter Number: 9601) as a wide-field reference image. A source catalog was created from the co-added MegaCam $r$ image, using the 2MASS catalog ${ }^{36}$ as an external reference catalog. The extracted $r$ catalog has been used as a reference for the SCAMP software (Bertin 2006) to derive an astrometric solution for the Suprime-Cam images.

\footnotetext{
32 http://smoka.nao.ac.jp

33 http://www.stsci.edu/ dcoe/trilogy/

34 IRAF is distributed by the National Optical Astronomy Observatory, which is operated by the Association of Universities for Research in Astronomy, Inc., under cooperative agreement with the National Science Foundation.

35 This research used facilities of the Canadian Astronomy Data Centre operated by the National Research Council of Canada with the support of the Canadian Space Agency.

36 This publication makes use of data products from the Two Micron All Sky Survey, which is a joint project of the University of Massachusetts and the Infrared Processing and Analysis Center/California Institute of Technology, funded by the National Aeronautics and Space Administration and the National Science Foundation.
} 

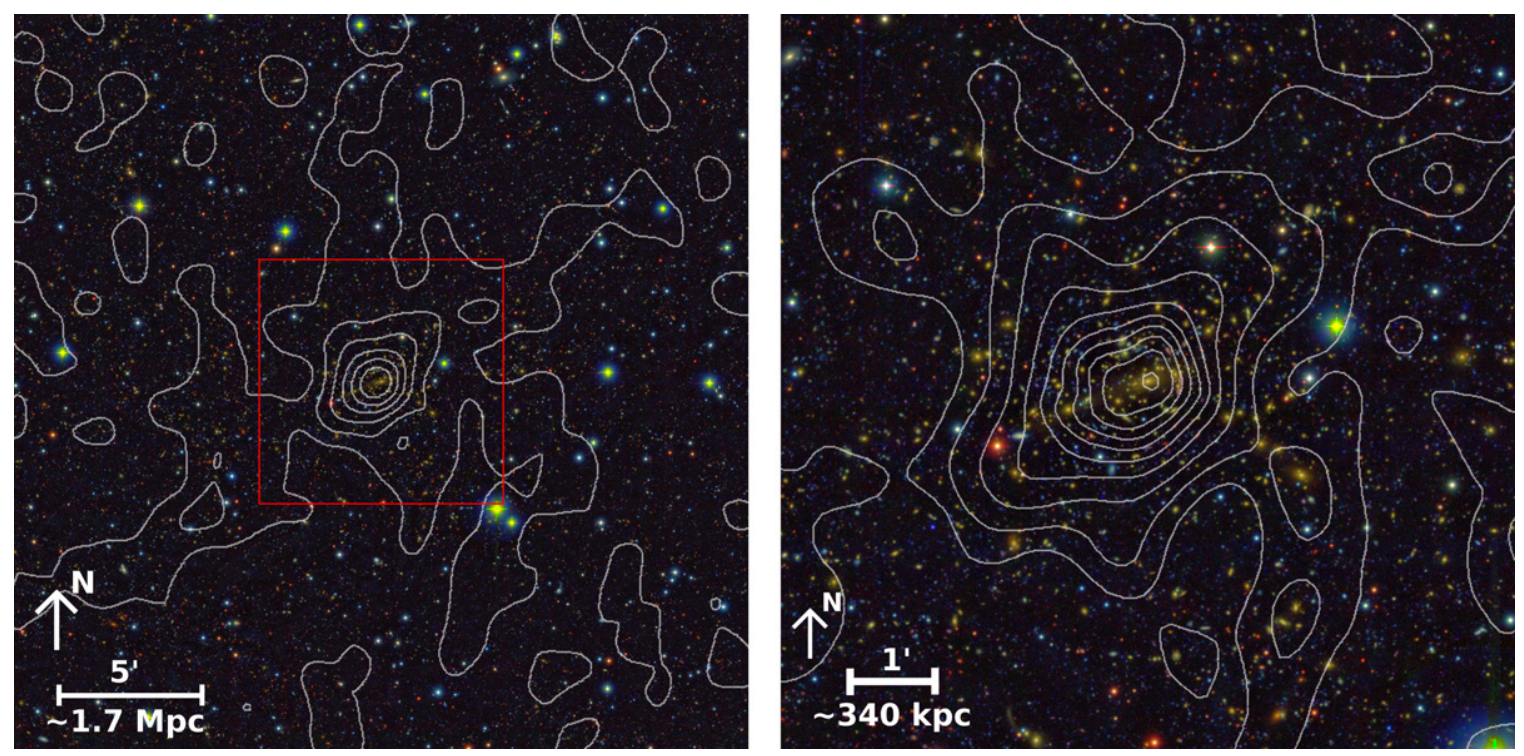

Figure 1. Subaru $B V R_{\mathrm{c}} I_{\mathrm{c}} z^{\prime}$ composite color images centered on the galaxy cluster MACS1206 $(z=0.439)$, overlaid with mass contours from our joint strong-and weak-lensing analysis (SAWLENS) of HST and Subaru observations. The image size in the left panel is $24^{\prime} \times 24^{\prime}$, covering a projected area of $5.7 \times 5.7 \mathrm{Mpc} h^{-2}$ at the cluster redshift. In the left and right panels, the lowest contour levels are $\kappa=0.12$ and 0.15 , with increments of $\Delta \kappa=0.09$ and 0.07 , respectively. The right panel is a zoom-in view of the boxed region of the left panel, with a side length of $8^{\prime}\left(1.9 \mathrm{Mpc} h^{-1}\right)$. North is top and east is left.

(A color version of this figure is available in the online journal.)

The photometric zero points for the co-added Suprime-Cam images were bootstrapped from a suitable set of reference stars identified in common with the calibrated MegaCam data. These zero points were refined in two independent ways: first by comparing cluster elliptical-type galaxies with the HST/ACS images, and subsequently by fitting SED (spectral energy distribution) templates with the BPZ code (Bayesian photometric redshift estimation; Benítez 2000; Benítez et al. 2004) to Subaru photometry of 1163 galaxies having measured spectroscopic redshifts from VLT/VIMOS (P. Rosati et al. 2012, in preparation). This leads to a final photometric accuracy of $\sim 0.01 \mathrm{mag}$ in all five passbands (see also Section 3.5). The five-band $B V R_{\mathrm{c}} I_{\mathrm{c}} z^{\prime}$ photometry catalog was then measured using SExtractor (Bertin \& Arnouts 1996) in point-spread function (PSF) matched images created by ColorPro (Coe et al. 2006), where a combination of all five bands was used as a deep detection image. The stellar PSFs were measured from a combination of 100 stars per band and modeled using IRAF routines.

For the weak-lensing shape analysis (Section 3.2), we use the $I_{\mathrm{c}}$-band data taken in 2009 January, which have the best image quality in our data sets (in terms of the stability and coherence of the PSF anisotropy pattern, taken in fairly good seeing conditions). Two separate co-added $I_{\mathrm{c}}$-band images, each with a total exposure time of $1.1 \mathrm{ks}$, were produced based on the imaging obtained at two different camera orientations separated by $90^{\circ}$, in order not to degrade the shape measurement quality.

\subsection{Subaru Weak-lensing Shape Analysis}

For shape measurements of background galaxies, we use our weak-lensing analysis pipeline based on the IMCAT package (Kaiser et al. 1995, hereafter KSB), incorporating modifications and improvements outlined in Umetsu et al. (2010). Our KSB+ implementation has been applied extensively to Subaru cluster observations (e.g., Broadhurst et al. 2005a, 2008; Umetsu et al. 2007, 2009, 2010, 2011a, 2011b; Umetsu \& Broadhurst 2008; Okabe \& Umetsu 2008; Medezinski et al. 2010, 2011; Zitrin et al. 2011c; Coe et al. 2012).
We measure components of the complex image ellipticity, $e_{\alpha}=\left\{Q_{11}-Q_{22}, Q_{12}\right\} /\left(Q_{11}+Q_{22}\right)$, from the weighted quadrupole moments of the surface brightness $I(\theta)$ of individual objects,

$$
Q_{\alpha \beta}=\int d^{2} \theta W(\theta) \theta_{\alpha} \theta_{\beta} I(\boldsymbol{\theta}) \quad(\alpha, \beta=1,2),
$$

where $W(\theta)$ is a Gaussian window function matched to the size $\left(r_{g}\right)$ of the object, and the weighted object centroid is chosen as the coordinate origin, which is iteratively refined to accurately measure the object shapes.

Next, we correct observed ellipticities $e_{\alpha}$ for the PSF anisotropy using a sample of stars in the field as references. We select bright $\left(18 \lesssim I_{\mathrm{c}} \lesssim 22\right)$, unsaturated stellar objects identified in a branch of the object half-light radius $\left(r_{h}\right)$ versus $I_{\mathrm{c}}$ diagram and measure the PSF anisotropy kernel of the KSB algorithm as a function of the object size $r_{g}$. Figure 2 shows the distributions of stellar ellipticity components $\left(e_{\alpha}^{*}\right)$ before and after the PSF anisotropy correction. From the rest of the object catalog, we select as a weak-lensing galaxy sample those objects with $v>10, r_{h}>\overline{r_{h}^{*}}+1.5 \sigma\left(r_{h}^{*}\right)$, and $r_{g}>\operatorname{mode}\left(r_{g}^{*}\right)$, where $v$ is the KSB detection significance and $\overline{r_{h}^{*}}$ and $\sigma\left(r_{h}^{*}\right)$ are median and rms dispersion values of stellar sizes $r_{h}^{*}$. The anisotropy-corrected ellipticities $e_{\alpha}^{\prime}$ are then corrected for the isotropic smearing effect as $g_{\alpha}=e_{\alpha}^{\prime} / P_{g}$.

For each galaxy we assign the statistical weight

$$
w_{(k)} \equiv \frac{1}{\sigma_{g(k)}^{2}+\alpha_{g}^{2}},
$$

where $\sigma_{g(k)}^{2}$ is the variance for the reduced shear estimate of the $k$ th galaxy computed from 50 neighbors identified in the $r_{g}-I_{\mathrm{c}}$ plane and $\alpha_{g}^{2}$ is the softening constant variance (e.g., Hamana et al. 2003; Umetsu \& Broadhurst 2008; Oguri et al. 2009; Okabe et al. 2010). This weighting scheme is essential to downweight faint and small objects that have noisy shape measurements (see Figure 4 of Umetsu et al. 2010). We choose $\alpha_{g}=0.4$, which is 


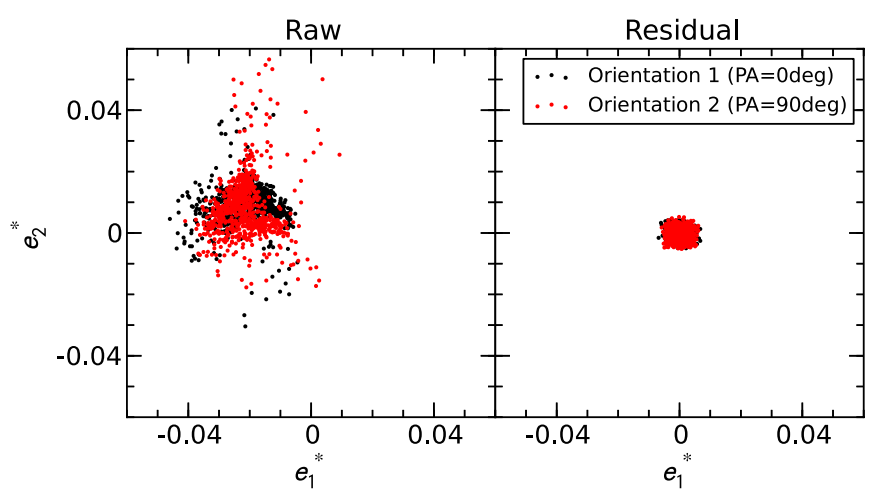

Figure 2. Stellar ellipticity distributions before and after the PSF anisotropy correction for Subaru/Suprime-Cam $I_{\mathrm{c}}$-band data taken with camera orientations of $\mathrm{PA}=0^{\circ}$ (Orientation 1; red) and $\mathrm{PA}=90^{\circ}$ (Orientation 2; black) The left panel shows the raw ellipticity components $\left(e_{1}^{*}, e_{2}^{*}\right)$ of stellar objects, and the right panel shows the residual ellipticity components $\left(\delta e_{1}^{*}, \delta e_{2}^{*}\right)$ after the PSF anisotropy correction.

(A color version of this figure is available in the online journal.)

a typical value of the mean rms $\sigma_{g}$ over the background sample (see Table 3; Umetsu \& Broadhurst 2008; Umetsu et al. 2009; Okabe et al. 2010).

\subsection{Shear Calibration}

We follow the shear calibration strategy of Umetsu et al. (2010) to improve the precision in shear recovery. This is motivated by the general tendency of $\mathrm{KSB}+$ to systematically underestimate the shear signal in the presence of measurement noise (see Umetsu et al. 2010; Okura \& Futamase 2012).

First, we select as a sample of shear calibrators those galaxies with $v>v_{c}$ and $P_{g}>0$. Here we take $v_{c}=20$. Note that the shear calibrator sample is a subset of the target galaxy sample. Second, we divide the calibrator $r_{g}-I_{\mathrm{c}}$ plane into a grid of $2 \times 10$ cells, each containing approximately equal numbers of calibrators, and compute a median value of $P_{g}$ at each cell. Then, each object in the target sample is matched to the nearest point on the $\left(r_{g}, I_{\mathrm{c}}\right)$ calibration grid to obtain a filtered measurement, $\left\langle P_{g}\right\rangle$. Finally, we use the calibrated estimator $g_{\alpha}=e_{\alpha}^{\prime} /\left\langle P_{g}\right\rangle$ for the reduced shear.

We have analyzed the two $I_{\mathrm{c}}$ mosaic images separately to construct a composite galaxy shape catalog, by properly weighting and combining the calibrated distortion measurements $\left(g_{\alpha}\right)$ for galaxies in the overlapping region.

We have tested our analysis pipeline using simulated Subaru Suprime-Cam images (see Section 3.2 of Oguri et al. 2012; Massey et al. 2007). We find that we can recover the weaklensing signal with good precision, typically, $|m| \lesssim 5 \%$ of the shear calibration bias, where the range of $m$-values shows a modest dependence of calibration accuracy on seeing conditions and PSF properties, and $c \sim 10^{-3}$ of the residual shear offset, which is about one order of magnitude smaller than the typical distortion signal in cluster outskirts $\left(|g| \sim 10^{-2}\right)$. This level of performance is comparable to other similarly well-tested methods (Heymans et al. 2006; Massey et al. 2007).

\subsection{Sample Selection}

A careful background selection is critical for a weak-lensing analysis so that unlensed cluster members and foreground galaxies do not dilute the true lensing signal of the background (Broadhurst et al. 2005a; Medezinski et al. 2007, 2010; Umetsu $\&$ Broadhurst 2008). This dilution effect is simply to reduce
Table 3

Galaxy Color Selection

\begin{tabular}{lrrrr}
\hline \hline Sample & $\begin{array}{c}\text { Magnitude Limits } \\
\text { (AB mag) }\end{array}$ & $N$ & $\begin{array}{c}n_{g}{ }^{\mathrm{b}} \\
\left(\mathrm{arcmin}^{-2}\right)\end{array}$ & $\left\langle z_{s}\right\rangle^{\mathrm{c}}$ \\
\hline Red & $21.5<z^{\prime}<24.6$ & 13252 & 9.9 & 1.16 \\
Green & $z^{\prime}<24.6$ & 1638 & 3.4 & 0.44 \\
Blue & $22.0<z^{\prime}<24.6$ & 4570 & 4.3 & 1.95 \\
\hline
\end{tabular}

Notes.

a Magnitude limits for the galaxy sample.

b Mean surface number density of source background galaxies.

${ }^{c}$ Mean photometric redshift of the sample obtained with the BPZ code.

the strength of the lensing signal when averaged over a local ensemble of galaxies (by a factor of $2-5$ at $R \lesssim 400 \mathrm{kpc} h^{-1}$; see Figure 1 of Broadhurst et al. 2005a), particularly at small cluster radius where the cluster is relatively dense, in proportion to the fraction of unlensed galaxies whose orientations are randomly distributed.

We use the background selection method of Medezinski et al. (2010) to define undiluted samples of background galaxies, which relies on empirical correlations for galaxies in color-color-magnitude space derived from the deep Subaru photometry, by reference to evolutionary tracks of galaxies (for details, see Medezinski et al. 2010; Umetsu et al. 2010), as well as to the deep photometric-redshift survey in the COSMOS field (Ilbert et al. 2009).

For MACS1206, we have a wide wavelength coverage $\left(B V R_{\mathrm{c}} I_{\mathrm{c}} z^{\prime}\right)$ of Subaru Suprime-Cam. We therefore make use of the $\left(B-R_{\mathrm{c}}\right)$ versus $\left(R_{\mathrm{c}}-z^{\prime}\right)$ color-color $(\mathrm{CC})$ diagram to carefully select two distinct background populations that encompass the red and blue branches of galaxies. We limit the data to $z^{\prime}=24.6 \mathrm{mag}$ in the reddest band, corresponding approximately to a $5 \sigma$ limiting magnitude within a $2^{\prime \prime}$ diameter aperture. Beyond this limit incompleteness creeps into the bluer bands, complicating color measurements, in particular of red galaxies.

To do this, we first identify in CC space an overdensity of galaxies with small projected distance $<3^{\prime}(\lesssim 1 \mathrm{Mpc}$ at $\left.z_{l}=0.439\right)$ from the cluster center. Then, all galaxies within this distinctive region define the green sample (see the green outlined region in Figure 3), comprising mostly the red sequence of the cluster and a blue trail of later type cluster members (Medezinski et al. 2010; Umetsu et al. 2010), showing a number density profile that is steeply rising toward the center (Figure 4, green crosses). The weak-lensing signal for this population is found to be consistent with zero at all radii (Figure 5, green crosses), indicating the reliability of our procedure. For this population of galaxies, we find a mean photometric redshift of $\left\langle z_{\text {phot }}\right\rangle \approx 0.44$ (see Section 3.5), consistent with the cluster redshift. Importantly, the green sample marks the region that contains a majority of unlensed galaxies, relative to which we select our background samples, as summarized below.

For the background samples, we define conservative color limits, where no evidence of dilution of the weak-lensing signal is visible, to safely avoid contamination by unlensed cluster members and foreground galaxies. The color boundaries for our blue and red background samples are shown in Figure 3. For the blue and red samples, we find a consistent, clearly rising weak-lensing signal all the way to the center of the cluster, as shown in Figure 5.

For validation purposes, we compare in $\mathrm{CC}$ space our color samples with a spectroscopic sample of cluster galaxies in 


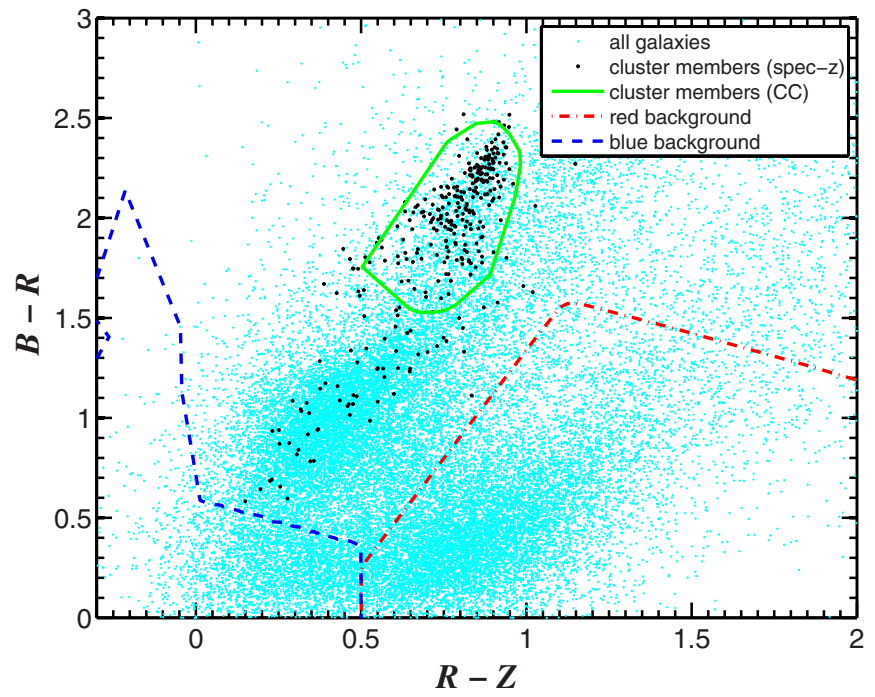

Figure 3. Blue and red background galaxies are selected for weak-lensing analysis (lower left blue dashed and right red dot-dashed regions, respectively) on the basis of Subaru $B R_{\mathrm{c}} z^{\prime}$ color-color-magnitude selection. All galaxies with $z^{\prime}<24.6$ mag (cyan) are shown in the diagram. At small radius, the cluster overdensity is identified as the green outlined region, defining our green sample comprising mostly the red sequence of the cluster and a blue trail of later type cluster members. The background samples are well isolated from the green region and satisfy other criteria as discussed in Section 3.4. Our background selection successfully excludes all spectroscopically confirmed cluster members (black) found within the projected cluster virial radius $\left(r_{\text {vir }} \approx 1.6 \mathrm{Mpc} h^{-1}\right)$. The cluster members are determined from the ongoing survey with VLT/VIMOS (P. Rosati et al. 2012, in preparation), using the algorithm of Mamon et al. (2010) in the dynamical analysis that will be presented in a forthcoming paper (A. Biviano et al. 2012, in preparation).

(A color version of this figure is available in the online journal.)

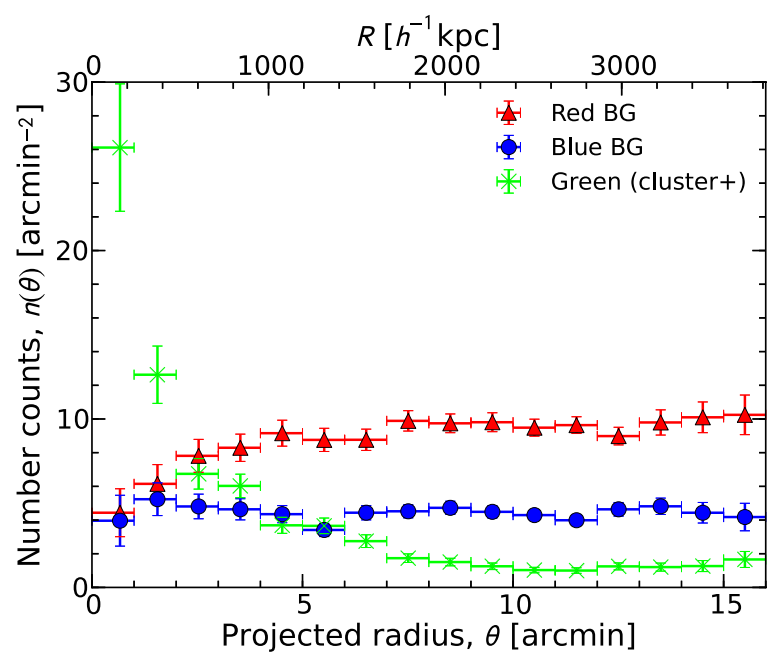

Figure 4. Surface number density profiles $n(\theta)$ of Subaru $B R_{\mathrm{c}} z^{\prime}$-selected galaxies used for the weak-lensing shape analysis. The results are shown for our red (triangles), blue (circles), and green (crosses) samples. See also Figure 9.

(A color version of this figure is available in the online journal.)

MACS1206. Figure 3 shows that the background selection procedure established in our earlier work (Medezinski et al. 2010, 2011; Umetsu et al. 2010) successfully excludes all spectroscopically confirmed cluster members found within the projected cluster virial radius $\left(r_{\mathrm{vir}} \approx 1.6 \mathrm{Mpc} h^{-1}\right.$; see Section 6). The cluster members are determined from the ongoing survey with VLT/VIMOS, part of the VLT-CLASH Large Programme 186.A-0798 (P. Rosati et al. 2012, in preparation), using the

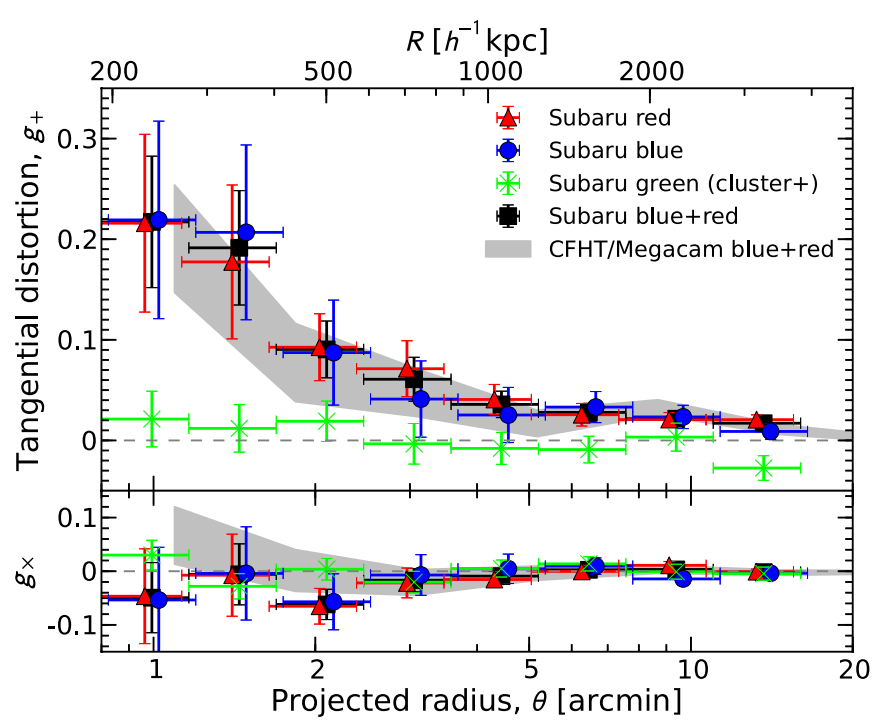

Figure 5. Azimuthally averaged radial profiles of the tangential reduced shear $g_{+}$(upper panel) and the $45^{\circ}$ rotated $(\times)$ component $g_{\times}$(lower panel) for our Subaru red (triangles), blue (circles), green (crosses), and blue+red (squares) galaxy samples shown in Figure 4. The error bars represent $68.3 \%$ confidence intervals estimated by bootstrap resampling techniques. The symbols for the red and blue samples are horizontally shifted for visual clarity. For a consistency check, we compare our Subaru results with CFHT/MegaCam data based on our $g r z$-selected background sample (gray area). The $g_{+}$profile for the green sample is consistent with a null signal at all radii, while this population is strongly clustered at small radius (Figures 4), indicating that the green galaxies mostly consist of cluster member galaxies. For all of the samples, the $\times$-component is consistent with a null signal detection well within $2 \sigma$ at all radii, indicating the reliability of our distortion analysis.

(A color version of this figure is available in the online journal.)

algorithm of Mamon et al. (2010) in the dynamical analysis that will be presented in a forthcoming paper (A. Biviano et al. 2012, in preparation). We find that about $70 \%$ of the cluster members overlap with our CC-selected green galaxies; the rest are cluster members with bluer colors. We note that there is a statistically inevitable fraction of interlopers even in the dynamically selected cluster membership as discussed in Wojtak et al. (2007, their Table 1) and Mamon et al. (2010, their Figure 13).

As a further consistency check, we also plot in Figure 4 the galaxy surface number density as a function of radius, $n(\theta)$, for the blue and red samples. As can be seen, no clustering is observed toward the center for the background samples, which demonstrate that there is no significant contamination by cluster members in the samples. The red sample reveals a systematic decrease in their projected number density toward the cluster center, caused by the lensing magnification effect (Section 2). A more quantitative magnification analysis is given in Section 5.2.2.

To summarize, our CC-selection criteria yielded a total of $N=13,252,1638$, and 4570 galaxies, for the red, green, and blue photometry samples, respectively (Table 3). For our weak-lensing distortion analysis, we have a subset of 8969 and 4154 galaxies in the red and blue samples (with usable $I_{\mathrm{c}}$ shape measurements), respectively (Table 4).

\subsection{Depth Estimation}

The lensing signal depends on the source redshift $z_{s}$ through the distance ratio $\beta\left(z_{s}\right)=D_{l s} / D_{s}$. We thus need to estimate and correct for the respective depths $\langle\beta\rangle$ of the different galaxy samples, when converting the observed lensing signal into physical mass units. 
Table 4

Galaxy Samples for Weak-lensing Shape Measurements

\begin{tabular}{|c|c|c|c|c|c|c|c|}
\hline \multirow[t]{2}{*}{ Sample } & \multirow[t]{2}{*}{$N$} & \multirow{2}{*}{$\begin{array}{c}n_{g}{ }^{\mathrm{a}} \\
\left(\operatorname{arcmin}^{-2}\right)\end{array}$} & \multirow[t]{2}{*}{$\sigma_{g}^{\mathrm{b}}$} & \multicolumn{2}{|c|}{$z_{s, \mathrm{eff}^{\mathrm{c}}}$} & \multicolumn{2}{|c|}{$\left\langle D_{l s} / D_{s}\right\rangle^{\mathrm{d}}$} \\
\hline & & & & M1206 & COSMOS & M1206 & COSMOS \\
\hline Red & 8969 & 9.2 & 0.42 & 1.05 & 1.05 & 0.51 & 0.51 \\
\hline Blue & 4154 & 4.3 & 0.48 & 1.55 & 1.58 & 0.62 & 0.63 \\
\hline Blue+red & 13123 & 13.4 & 0.44 & 1.15 & 1.12 & 0.54 & 0.53 \\
\hline
\end{tabular}

Notes.

${ }^{a}$ Mean surface number density of source background galaxies.

${ }^{\mathrm{b}}$ Mean rms error for the shear estimate per galaxy, $\sigma_{g} \equiv\left(\overline{\sigma_{g}^{2}}\right)^{1 / 2}$

${ }^{\mathrm{c}}$ Effective source redshift corresponding to the mean depth $\langle\beta\rangle$ of the sample.

${ }^{\mathrm{d}}$ Distance ratio averaged over the redshift distribution of the sample, $\langle\beta\rangle$.

For this we used BPZ (Section 3.1) to measure photometric redshifts (photo- $z \mathrm{~s}) z_{\text {phot }}$ for our deep Subaru $B V R_{\mathrm{c}} I_{\mathrm{c}} z^{\prime}$ photometry (Section 3.1). BPZ employs a Bayesian inference where the redshift likelihood is weighted by a prior probability, which yields the probability density $P(z, T \mid m)$ of a galaxy with apparent magnitude $m$ of having certain redshift $z$ and spectral type $T$. In this work we used a new library (N. Benitez 2012, in preparation) composed of 10 SED templates originally from PEGASE (Fioc \& Rocca-Volmerange 1997) but recalibrated using the FIREWORKS photometry and spectroscopic redshifts from Wuyts et al. (2008) to optimize its performance. This library includes five templates for elliptical galaxies, two for spiral galaxies, and three for starburst galaxies. In our depth estimation we utilize BPZ's ODDS parameter, which measures the amount of probability enclosed within a certain interval $\Delta z$ centered on the primary peak of the redshift probability density function (pdf), serving as a useful measure to quantify the reliability of photo- $z$ estimates (Benítez 2000). ${ }^{37}$ We used our VLT/VIMOS sample of 1163 galaxies with spectroscopic redshifts $z_{\text {spec }}(\lesssim 1.5)$ to assess the performance of our photo- $z$ estimation. From the whole sample, we find an rms scatter of $\sigma\left(\delta_{z}\right) \approx 0.027$ in the fractional error $\delta_{z} \equiv\left(z_{\text {phot }}-z_{\text {spec }}\right) /\left(1+z_{\text {spec }}\right)$, with a small mean offset $\mu\left(\delta_{z}\right)=-0.0021$ and a $5 \sigma$ outlier fraction of $\approx 5.5 \%$. Using a subsample of $\sim 510$ galaxies with $0.3<z_{\text {spec }}<0.5$, we find $\sigma\left(\delta_{z}\right) \approx 0.031$ with $\approx 1.5 \%$ of outliers.

For a consistency check, we also make use of the COSMOS catalog (Ilbert et al. 2009) with robust photometry and photo- $z$ measurements for the majority of galaxies with $i^{\prime}<25$ mag. For each sample, we apply the same CC selection to the COSMOS photometry and obtain the redshift distribution $N(z)$ of field galaxies.

For each background population, we calculate weighted moments of the distance ratio $\beta$ as

$$
\left\langle\beta^{n}\right\rangle=\frac{\int d z w(z) N(z) \beta^{n}(z)}{\int d z w(z) N(z)},
$$

where $w(z)$ is a weight factor, $w$ is taken to be the Bayesian ODDS parameter for the BPZ method, and $w=1$ otherwise. The sample mean redshift $\left\langle z_{s}\right\rangle$ is defined similarly to Equation (10). The first moment $\langle\beta\rangle$ represents the mean lensing depth. ${ }^{38}$ It is useful to define the effective single-plane source

\footnotetext{
37 In the present work, we set $\Delta z=2 \times 0.03\left(1+z_{\text {phot }}\right)$, which is approximately twice the width $(\sigma)$ of the error distribution.

38 In general, a wide spread of the redshift distribution of background galaxies, in conjunction with the single-plane approximation, may lead to an overestimate of the gravitational shear in the nonlinear regime (Hoekstra et al. 2000). Thanks to the deep Subaru photometry, we found that this bias in the observed reduced shear is approximately $\Delta g / g \approx\left(\left\langle\beta^{2}\right\rangle /\langle\beta\rangle^{2}-1\right) \kappa \approx 0.06 \kappa$ to the first order of $\kappa$. See Section 3.4 of Umetsu et al. (2010) for details.
}

redshift, $z_{s, \text { eff }}$, such that (Umetsu \& Broadhurst 2008; Umetsu et al. 2009, 2010)

$$
\beta\left(z_{s, \mathrm{eff}}\right)=\langle\beta\rangle .
$$

In Table 4 we summarize the mean depths $\langle\beta\rangle$ and the effective source redshifts $z_{s, \text { eff }}$ for our background samples. For each background sample, we obtained consistent mean-depth estimates $\langle\beta\rangle$ (within 2\%) using the BPZ- and COSMOS-based methods. In the present work, we adopt a conservative uncertainty of $5 \%$ in the mean depth for the combined blue and red sample of background galaxies, $\langle\beta$ (back) $\rangle=0.54 \pm 0.03$, which corresponds to $z_{s, \text { eff }}=1.15 \pm 0.1$. We marginalize over this uncertainty when fitting parameterized mass models to our weak-lensing data.

\section{CLUSTER STRONG-LENSING ANALYSIS}

For a massive cluster, the strong- and weak-lensing regimes contribute quite similar logarithmic coverage of the radial mass profile. It is therefore crucial to include the central stronglensing information in a cluster lensing analysis (e.g., Umetsu et al. 2011a, 2011b).

Here we perform several complementary strong-lensing analyses using a wide variety of modeling methods, namely, the Zitrin et al. (2009) method, Lenstool (Kneib et al. 1996; Jullo et al. 2007), LensPerfect (Coe et al. 2010, 2012), Pixelens (Saha \& Williams 2004; Grillo et al. 2010), and a joint strongand weak-lensing reconstruction method of Merten et al. (2009, 2011) (hereafter SAWLENS). All analyses here use the positions and redshifts of multiply lensed images identified by Zitrin et al. (2012).

Lens reconstruction methods are broadly classified into parametric and non-parametric. In the former approach, the total mass distribution of the deflector is described in terms of a set of theoretically (and/or observationally) motivated models, each specified by a particular functional form characterized by a small number of free parameters. This involves, to some extent, the assignment of halos to visible galaxies assuming that light approximately traces mass, while the latter does not except for certain priors on the mass distribution. ${ }^{39}$ Among the methods used in the present work, the Zitrin et al. (2009) method and Lenstool are parametric; LensPerfect, Pixelens, and SAWLENS are non-parametric.

For this work, we primarily use the detailed strong-lens modeling of Zitrin et al. (2012) based on deep CLASH imaging and VLT/VIMOS spectroscopy, as summarized in Section 4.1. The cluster miscentering effects are discussed in Section 4.2. In Section 4.3 we introduce and apply a technique to selfcalibrate the bin-bin covariance matrix of the central radial mass profile derived from the reanalysis of Zitrin et al. (2012). In Section 4.4 we perform several semi-independent stronglensing analyses on the MACS1206 HST images, utilizing various modeling methods, in order to verify the identifications of the multiple images and to independently assess the level of inherent systematic uncertainties in our analyses.

\subsection{Primary Strong-lensing Model}

Here we briefly summarize our well-tested approach to strong-lens modeling, developed by Broadhurst et al. (2005b)

\footnotetext{
39 The latter is often based on the assumption that the lens profiles and/or distributions can be well approximated by a pixelated mass distribution (e.g., PiXelens and SAWLENS).
} 
and optimized further by Zitrin et al. (2009), which has previously uncovered large numbers of multiply lensed galaxies in HST images of many clusters (e.g., Broadhurst et al. 2005b; Zitrin et al. 2009, 2010, 2011a, 2011b, 2011c). In the present work, we use a new Markov Chain Monte Carlo (MCMC) implementation of the Zitrin et al. (2009) method, where also the BCG mass is allowed to vary. ${ }^{40}$

Ourflexible mass model consists of four components, namely, the BCG, cluster galaxies, a smooth DM halo, and the overall matter ellipticity (corresponding to a coherent external shear; for details, see Zitrin et al. 2009), described by seven free parameters in total. ${ }^{41}$ The basic assumption adopted is that cluster galaxy light approximately traces the DM; the latter is modeled as a smoothed version of the former (see, for details, Zitrin et al. 2009). This approach to strong lensing is sufficient to accurately predict the locations and internal structure of multiple images, since in practice the number of multiple images uncovered readily exceeds the number of free parameters, so that the fit is fully constrained.

Zitrin et al. (2012) identified 47 new multiple images of 12 distant sources (including three candidate systems; Systems 9-11 therein), in addition to the known giant arc system at $z_{s}=1.03$ (Ebeling et al. 2009), bringing the total known for this cluster to 50 multiply lensed images of 13 sources, spanning a wide redshift range of $1 \lesssim z_{s} \lesssim 5.5$, spread fairly evenly over the central region, $3^{\prime \prime} \lesssim \theta \lesssim 1^{\prime}$. Zitrin et al. (2012) used the position and redshift of 32 secure multiple images of nine systems to constrain the mass model. Following Zitrin et al. (2012), we adopt an image positional error of $2^{\prime \prime}\left(\approx 1^{\prime \prime} .4\right.$ in each dimension), which is a typical value in the presence of uncorrelated large-scale structure (LSS) along the line of sight (for details, see Zitrin et al. 2012; Host 2012; Jullo et al. 2010). Including the BCG mass as an additional free parameter, we find here an acceptable fit with the minimized $\chi^{2}$ value $\left(\chi_{\min }^{2}\right)$ of 22.8 for 39 dof, with an image-plane reproduction error of 1".76. The new MCMC results are in good agreement with the results of Zitrin et al. (2012), as shown here in Figure 6, with only some minor differences at the innermost radii $\lesssim 2^{\prime \prime}\left(\sim 8 \mathrm{kpc} h^{-1}\right)$ dominated by the BCG (see Newman et al. 2009). The detailed central mass map reveals a fairly elliptical outer critical curve (see Figure 1 of Zitrin et al. 2012). For a source at $z_{s}=2.54$, the outer critical curve encloses an area with an effective Einstein radius of $\theta_{\text {Ein }}=28^{\prime \prime} \pm 3^{\prime \prime}$; for the lower-redshift system with $z_{s}=1.03$, the effective Einstein radius of the critical area is $\theta_{\text {Ein }}=17^{\prime \prime} \pm 3^{\prime \prime}$ (Table 1).

\subsection{Cluster Miscentering Effects}

To obtain meaningful radial profiles, one must carefully define the cluster center. It is often assumed that the cluster mass centroid coincides with the BCG position, whereas BCGs can be offset from the mass centroids of the corresponding DM halos (Johnston et al. 2007; Oguri \& Takada 2011; Umetsu et al. 2011a, 2011b).

Here we utilize our detailed mass model of Zitrin et al. (2012), which allows us to locate the peak position of the smooth DM component, providing an independent mass centroid determination (e.g., Umetsu et al. 2010, 2011b). In this method, we

\footnotetext{
$\overline{40}$ Our very preliminary MCMC results were presented in Figure 4 of Zitrin et al. (2012).

41 The Zitrin et al. (2009) method employs grid-based maximum-likelihood parameter estimation in the six-dimensional parameter space, where the seventh parameter included in the present work is the BCG mass.
}

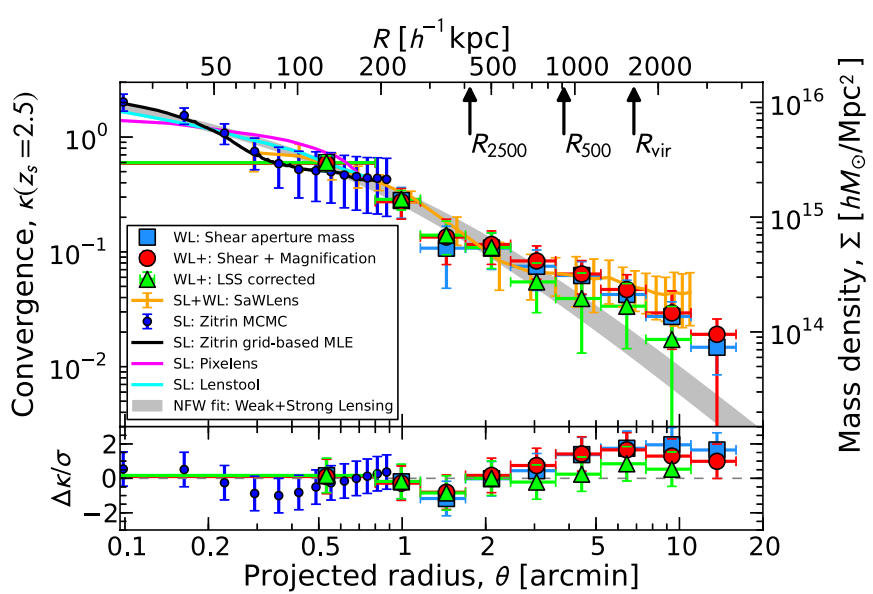

Figure 6. Surface-mass density profile $\kappa$ derived from our Subaru weak-lensing and Hubble strong-lensing measurements. The red circles represent our full weak-lensing constraints from joint shear and magnification measurements (Figure 9), consistent with the purely shear-based results (squares) and the SAWLENS results (orange line with error bars), all showing a shallow radial trend with a nearly isothermal logarithmic density slope, $d \ln \Sigma / d \ln R \sim-1$. For weak lensing, the innermost bin represents the average convergence $\bar{\kappa}\left(<\theta_{\min }\right)$ interior to the inner radial boundary of the weak-lensing data $\left(0,8 \leqslant \theta \leqslant 16^{\prime}\right)$, $\theta_{\min }=0.8$, which is about twice the Einstein radius for a distant background source at $z_{s} \sim 2$ (see Tables 3 and 4) and hence sufficiently large for our background galaxies at an effective source redshift of $z_{s, \text { eff }}=1.15 \pm 0.1$. The triangles show the NE-SW mass profile excluding the large-scale structure extending along the NW-SE direction (see Figure 8), derived from a twodimensional mass reconstruction using both shear and magnification data, in good agreement with the standard NFW form (gray area). The black solid line is the best-fit model of Zitrin et al. (2012) based on the grid-based maximumlikelihood parameter estimation. The small blue circles with error bars represent our primary strong-lens constraints on the binned mass profile derived from an MCMC implementation of Zitrin et al. (2012). The errors are based on the selfcalibrated covariance matrix (only every other point is shown here; Section 4.3). Our mass profile results from several weak- and strong-lensing methods all agree in the regions of overlap within their corresponding uncertainties. For the sake of clarity, the Pixelens and Lenstool results are shown without error bars. The bottom panel shows the respective deviations $\Delta \kappa$ (in units of the error $\sigma$ ) from the best-fit NFW model. The projected mass profile averaged over all azimuthal angles (squares, circles) shows a systematic excess at large radii with $R \gtrsim 1 \operatorname{Mpc}^{-1}\left(\theta \gtrsim 4^{\prime}\right)$.

(A color version of this figure is available in the online journal.)

approximate the large-scale distribution of cluster mass by assigning a power-law mass profile to each cluster galaxy, the sum of which is then smoothed to represent the DM distribution. The success of this simple model in describing the projected mass distributions of lensing clusters, as well as identifying many sets of multiply lensed images, assures us that the effective DM center can be determined using multiple images and the distribution of cluster member galaxies. In this context, the DM peak location is primarily sensitive to the degree of smoothing $(S)$ and the index of the power law ( $q$ ) of Zitrin et al. (2009).

We find only a small offset of $\sim 1^{\prime \prime}$, or a projected offset distance of $d_{\text {off }}=4 \mathrm{kpc} h^{-1}$ at $z_{l}=0.439$, between the BCG and the DM peak of mass, well within the uncertainties. The BCG position also coincides well with the peak of X-ray emission within $2^{\prime \prime}$ in projection (Table 1). This level of cluster centering offset is fairly small as compared to those found in other highmass clusters, say, $d_{\text {off }} \approx 20 \mathrm{kpc} h^{-1}$ in RX J1347-11 (Umetsu et al. 2011b), often implied by other massive bright galaxies in the vicinity of the BCG. In the present work, we thus adopt the BCG position as the cluster center and limit our analysis to radii greater than $4^{\prime \prime}\left(\approx 16 \mathrm{kpc} h^{-1}\right)$, which is approximately the location of the innermost strong-lensing constraint (see Section 4.1) and sufficiently large to avoid the BCG contribution. 
This inner radial limit corresponds roughly to $4 d_{\text {off }}\left(>2 d_{\text {off }}\right)$, beyond which smoothing from the cluster miscentering effects on the $\Sigma$ profile is sufficiently negligible (Johnston et al. 2007; Umetsu et al. 2011a; Sereno \& Zitrin 2012).

\subsection{Self-calibration of the Covariance Matrix}

The MCMC approach allows for a full parameter-space extraction of the underlying lensing signal. We construct from MCMC samples a central mass profile $\kappa_{i}$ and its covariance matrix $\mathcal{C}_{i j}$ in linearly spaced radial bins, spanning from $\theta=1^{\prime \prime}$ to the limit of our ACS data, $\theta \sim 100^{\prime \prime}$. Note that multiple-image constraints are available out to a radius of $\approx 1^{\prime}$ (Section 4.1 ), so that the mass model beyond this radius is constrained essentially by the light distribution of cluster member galaxies, and hence the constraints there are driven by the prior. We find that the mass profile is positively correlated from bin to bin, especially at radii beyond $\theta_{\text {Ein }} \approx 28^{\prime \prime}\left(z_{s}=2.5\right)$. Accordingly, the $\mathcal{C}$ matrix is nearly singular, with very small eigenvalues associated with large-scale modes where the constraints are weaker, leading to underestimated diagonal errors at $\theta \gtrsim \theta_{\text {Ein }} \approx 28^{\prime \prime}\left(z_{s}=2.5\right)$.

Here, we use a regularization technique with a single dof to calibrate the $\mathcal{C}$ matrix and obtain conservative errors for strong lensing, accounting for possible systematic errors introduced by the prior assumptions in the modeling. We first perform an eigenvalue decomposition as $\mathcal{C}=U \Lambda U^{t}$, where $\Lambda$ is a diagonal matrix of eigenvalues and $U$ is a unitary matrix of eigenvectors. Then, we determine our regularization constant, the minimum eigenvalue $\Lambda_{\min }$, by conservatively requiring that the outermost $\kappa$ value, $\kappa_{\min }=\kappa\left(100^{\prime \prime}\right) \approx 0.22$, is consistent with a null detection, i.e., $\Lambda_{\min }=\kappa_{\min }^{2}=(0.22)^{2}$. Replacing those less than $\Lambda_{\min }$ by $\Lambda_{\min }$ and restoring the $\mathcal{C}$ matrix with the regularized $\Lambda$ yields the desired, self-calibrated $\mathcal{C}$ matrix. All points at $\gtrsim 1^{\prime}$ are then excluded from our analysis. We find that a weaker regularization with $\Lambda_{\min }=(0.1)^{2}$ only affects the halo parameters $\left(M_{\mathrm{vir}}, c_{\mathrm{vir}}\right)$ by less than $4 \%$.

In Figure 6 we show our strong-lensing constraints on the central $\kappa$ profile using the self-calibrated $\mathcal{C}$ matrix, where the outer radial boundary is conservatively set to $\theta=53^{\prime \prime}\left(\approx 2 \theta_{\text {Ein }}\right.$ at $z_{s}=2$; see Zitrin et al. 2012). This calibration scheme produces conservative error estimates. Overall, the level of correction applied to the $\mathcal{C}$ matrix increases with increasing radius. We introduce here an estimator for the total signal-to-noise ratio $(\mathrm{S} / \mathrm{N})$ for detection, integrated over the radial range considered, and quantify the significance of the reconstruction, by the following equation (Umetsu \& Broadhurst 2008):

$$
(\mathrm{S} / \mathrm{N})^{2}=\sum_{i, j} \kappa_{i} \mathcal{C}_{i j}^{-1} \kappa_{j}=\boldsymbol{\kappa}^{t} \mathcal{C}^{-1} \boldsymbol{\kappa} .
$$

With the calibrated $\mathcal{C}$ matrix, we find a total $\mathrm{S} / \mathrm{N}$ of $\approx 18$ for our strong-lensing $\kappa$ profile in the radial range $\theta \leqslant 53^{\prime \prime}$. We check that our results are insensitive to the choice of radial binning scheme when the self-calibration technique is applied.

\subsection{Complementary Strong-lensing Analyses}

We have performed complementary semi-independent strong-lensing analyses (Lenstool, LensPerfect, Pixelens, SAWLENS), using as input the sets (or subsets) of multiple images identified by Zitrin et al. (2012) and the same spectroscopic and photometric redshift information.

In our LENSTOOL analysis, we parameterize the lens mass distribution $\Sigma(\boldsymbol{\theta})$ as a multi-component model consisting of an elliptical NFW potential and truncated elliptical halos (Kassiola
\& Kovner 1993) for the 86 brightest cluster members. All nine of the secure image systems are included as observational constraints. Our best solution reproduces all arc systems included and the critical lines at $z_{s}=2.54$ and 1.03 derived in Zitrin et al. (2012), with an image-plane rms of 1.9 , very similar to the value of $\sim 1^{\prime \prime}$. 8 obtained by Zitrin et al. (2012) and typical to parametric mass models for clusters with many multiple images (Broadhurst et al. 2005b; Halkola et al. 2006; Limousin et al. 2007; Zitrin et al. 2009).

In the PIXELENS analysis we model the lens mass distribution on a circular grid of $52^{\prime \prime}$ radius divided into 18 pixels. We consider 200 models with decreasing projected mass profiles (i.e., $\Sigma(R) \propto R^{-\alpha}$ with $\alpha>0$ ). We use as constraints the spectroscopically confirmed Systems 1-4 (Zitrin et al. 2012) of 14 multiple images, spanning the range $3.5-46^{\prime \prime}$ in radius. We check that adding other multiple-image systems identified in Zitrin et al. (2012) does not significantly affect the PIXELENS mass reconstruction.

In the LENSPERFECT analysis, we assume a prior that the projected mass is densest near the center of the BCG and decreases outward. Other priors include overall smoothness and approximate azimuthal symmetry (for details, see Coe et al. 2010). All secure image systems are used in this modeling, where including the three candidate systems (9-11) does not change the results significantly.

The SAWLENS method combines central strong-lensing constraints from multiple-image systems with weak-lensing distortion constraints in a non-parametric manner to reconstruct the underlying lensing potential on an adaptively refined mesh. For this cluster we use two levels of refinement, providing a 6" pixel resolution in the strong-lensing regime covered by CLASH imaging and $\mathrm{a} \approx 22^{\prime \prime}$ resolution in the Subaru weak-lensing field where the background source galaxies are sparsely sampled. The field size for the reconstruction is $25^{\prime}$ on a side. All image systems except 10 and 11 are included as strong-lensing constraints. The lens distortion measurements for the blue+red sample are used as weak-lensing constraints. The reconstruction errors are derived from 1000 bootstrap realizations of the weak-lensing background catalog and 1000 samples of the redshift uncertainties in the catalog of strong-lensing features. The number of realizations is limited by runtime constraints.

Figure 7 shows and compares the resulting projected integrated mass profiles $M_{2 \mathrm{D}}(<\theta)$ derived from our comprehensive strong-lensing analyses, along with our primary strong-lensing results and model-independent Einstein-radius constraints based on Zitrin et al. (2012). All these models are broadly consistent with the Einstein-radius constraints. The calibrated error bars of Zitrin et al. (2012) are roughly consistent with the spread of the semi-independent mass profiles derived here. This comparison shows clear consistency among a wide variety of analysis methods with different assumptions and systematics, which firmly supports the reliability of our strong-lensing analyses and calibration.

\section{CLUSTER WEAK-LENSING ANALYSIS}

This section is devoted to our cluster weak-lensing analysis based on the deep multi-color Subaru observations. In Section 5.1 we present the projected mass and galaxy distributions in and around MACS1206. In Section 5.2 we derive cluster lens distortion and magnification radial profiles from Subaru data. In Section 5.3 we briefly summarize our Bayesian mass inversion methods based on combined lens distortion and 


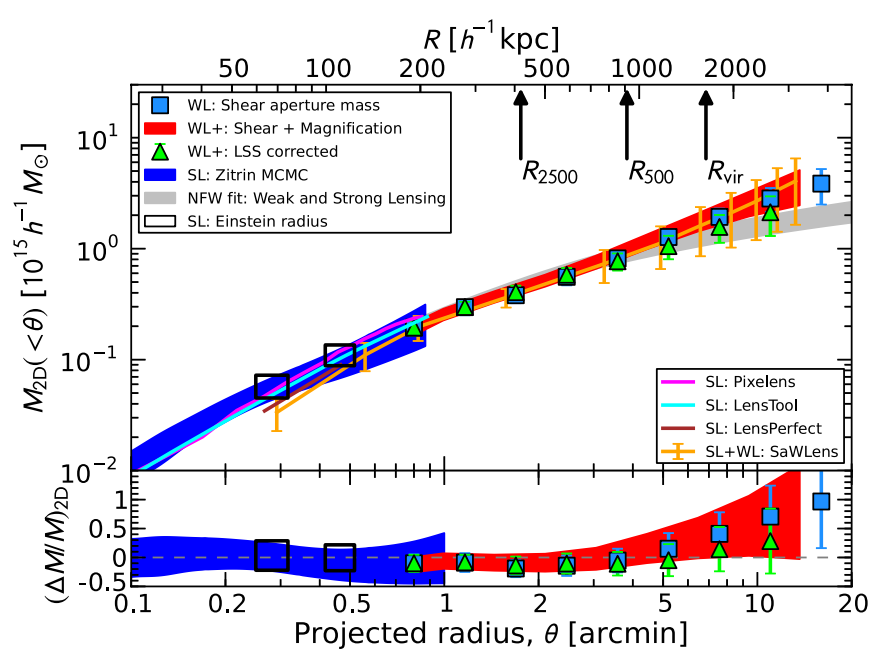

Figure 7. Comparison of projected cumulative mass profiles $M_{2 \mathrm{D}}$ of MACS1206 obtained with different lensing methods. The red shaded area shows our full weak-lensing constraints $(68 \% \mathrm{CL})$ derived from a joint Bayesian analysis of Subaru shear and magnification measurements (Figure 9), in good agreement with the shear aperture mass measurements (squares) obtained with a zerodensity boundary condition of $\bar{\Sigma}\left(16^{\prime}<\theta<18^{\prime}\right)=0$. The triangles denote the mass profile using the NE-SW $\Sigma$ profile of Figure 6 excluding the NW-SE excess regions. The two open rectangles represent model-independent Einsteinradius constraints of $\theta_{\text {Ein }}=17^{\prime \prime} \pm 2^{\prime \prime}\left(z_{s}=1.03\right)$ and $\theta_{\text {Ein }}=28^{\prime \prime} \pm 3^{\prime \prime}$ $\left(z_{s}=2.54\right)$. The blue shaded area represents our primary strong-lens model with $1 \sigma$ uncertainty from an MCMC implementation of Zitrin et al. (2012), which is broadly consistent with our semi-independent results from a wide variety of four strong-lens modeling analyses (Pixelens, Lenstool, LensPerfect, and SAWLENS), providing a valuable consistency check. Our independent strongand weak-lensing profiles are in good agreement in the region of overlap and together are well described by the standard NFW form (gray area), but they increasingly exceed it at $R \gtrsim 1 \mathrm{Mpc} h^{-1}$ out to the limit of our data. The bottom panel shows fractional deviations $(\Delta M / M)_{2 \mathrm{D}}$ of projected mass profiles with respect to the best-fit NFW model (top, gray), demonstrating the presence of a large-scale anisotropy in the mass distribution around the cluster.

(A color version of this figure is available in the online journal.)

magnification measurements and apply to Subaru weak-lensing observations of MACS1206.

\subsection{Two-dimensional Mass Map}

Weak-lensing distortion measurements $(g)$ can be used to reconstruct the underlying projected mass density field $\Sigma(\boldsymbol{\theta})$ (see Equation (3)). Here we use the linear map-making method outlined in Section 4.4 of Umetsu et al. (2009) to derive the projected mass distribution from the Subaru distortion data presented in Section 3.

In the left panel of Figure 8 , we show the $\Sigma(\boldsymbol{\theta})$ field in the central $24^{\prime} \times 24^{\prime}$ region, reconstructed from the blue+red sample (Section 3.4), where for visualization purposes the mass map is smoothed with a Gaussian with 1'.5 FWHM. A prominent mass peak is visible in the cluster center. This first maximum in the mass map is detected at a significance level of $9.5 \sigma$ and coincides well with the optical/X-ray cluster center within the statistical uncertainty: $\Delta$ R.A. $=7^{\prime \prime} .0 \pm 7^{\prime \prime} .2, \Delta$ decl. $=$ $-1^{\prime \prime} .4 \pm 7$.' 6 , where $\Delta$ R.A. and $\Delta$ decl. are right ascension and declination offsets, respectively, from the BCG center.

Also compared in Figure 8 are member galaxy distributions in the MACS1206 field, Gaussian smoothed to the same resolution of $\theta_{\text {FWHM }}=1.5$. The middle and right panels display the number and ( $K$-corrected) $R_{\mathrm{c}}$-band luminosity density fields, respectively, of green cluster galaxies (see Table 3 ).

Overall, mass and light are similarly distributed in the cluster. The cluster is fairly centrally concentrated in projection and associated with elongated LSS running northwest-southeast (NW-SE), both in the projected mass and galaxy distributions. A more quantitative characterization of the 2D matter distribution around the cluster will be given in Section 6 .

\subsection{Cluster Weak-lensing Profiles}

Now we derive azimuthally averaged lens distortion and magnification profiles from the Subaru data. We calculate the weaklensing profiles in $N$ discrete radial bins from the cluster center (Section 4.2), spanning the range $\left[\theta_{\min }, \theta_{\max }\right]$ with a constant logarithmic radial spacing $\Delta \ln \theta=\ln \left(\theta_{\max } / \theta_{\min }\right) / N$, where the inner radial boundary $\theta_{\min }$ is taken to be $\theta_{\min }=0.8$ ( $\left.>\theta_{\text {Ein }}\right)$. The outer radial boundary $\theta_{\max }$ is chosen to be $\theta_{\max }=16^{\prime}$
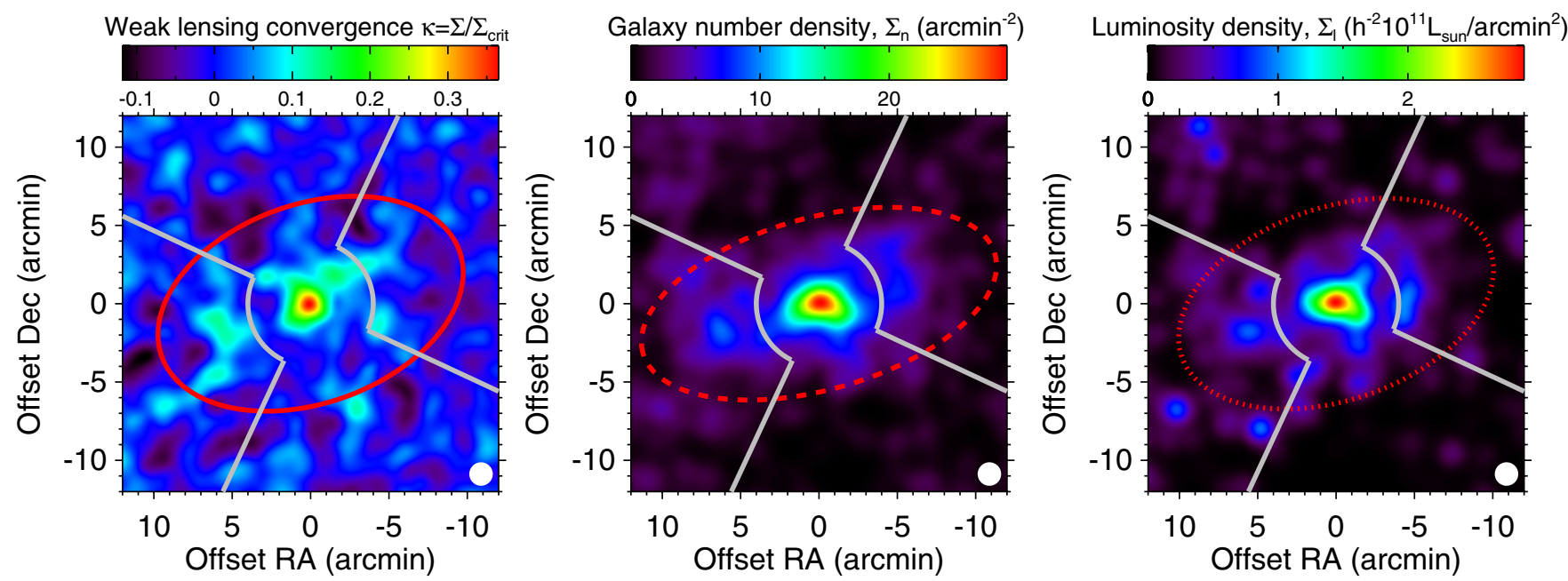

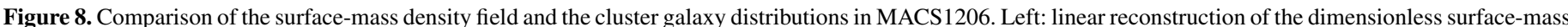

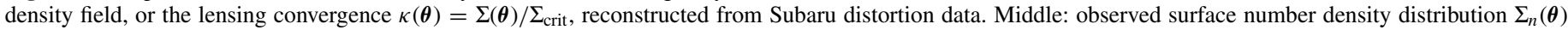

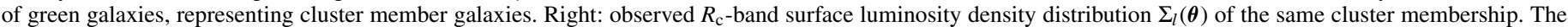

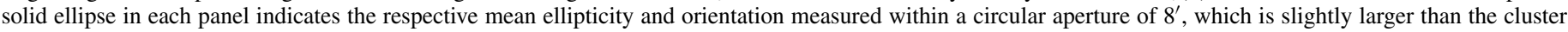

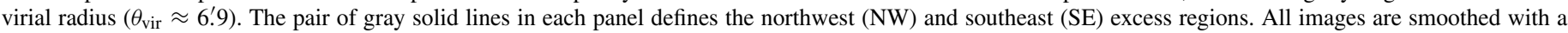
circular Gaussian of FWHM 1'.5. The field size is $24^{\prime} \times 24^{\prime}$. North is to the top, east to the left.

(A color version of this figure is available in the online journal.) 
$\left(R_{\max } \approx 3.8 \mathrm{Mpc} h^{-1}\right)$, sufficiently larger than the typical virial radius $r_{\text {vir }}$ of high-mass clusters $\left(r_{\text {vir }} \approx 1.6 \mathrm{Mpc} h^{-1}\right.$ for MACS1206; see Section 6), but sufficiently small with respect to the size of the Suprime-Cam's field of view so as to ensure accurate PSF anisotropy correction. The number of radial bins is set to $N=8$, chosen such that the detection $\mathrm{S} / \mathrm{N}$ (defined as in Equation (12)) is of the order of unity per pixel.

\subsubsection{Lens Distortion}

For each galaxy, we define the tangential distortion $g_{+}$and the $45^{\circ}$ rotated component, with respect to the cluster center, from linear combinations of the distortion coefficients $\left(g_{1}, g_{2}\right)$ as $g_{+}=$ $-\left(g_{1} \cos 2 \phi+g_{2} \sin 2 \phi\right)$ and $g_{\times}=-\left(g_{2} \cos 2 \phi-g_{1} \sin 2 \phi\right)$, with $\phi$ being the position angle of an object with respect to the cluster center. In the absence of higher-order effects, weak lensing only induces curl-free tangential distortions, while the azimuthal averaged $\times$ component is expected to vanish. In practice, the presence of $\times$ modes can be used to check for systematic errors.

For each galaxy sample, we calculate the weighted average of $g_{+}$in a set of radial bins $(i=1,2, \ldots, N)$ as

$$
g_{+, i} \equiv g_{+}\left(\theta_{i}\right)=\left[\sum_{k \in i} w_{(k)} g_{+(k)}\right]\left[\sum_{k \in i} w_{(k)}\right]^{-1},
$$

where the index $k$ runs over all objects located within the $i$ th annulus, $\theta_{i}$ is the weighted center of the $i$ th radial bin, and the weight factor $w_{(k)}$ is defined by Equation (9). We use the continuous limit of the area-weighted center for $\theta_{i}$ (see Appendix A of Umetsu \& Broadhurst 2008). We perform a bootstrap error analysis to assess the uncertainty $\sigma_{+, i}$ in the tangential distortion profile $g_{+, i}$ (Umetsu et al. 2010).

In Figure 5, we compare azimuthally averaged radial profiles of $g_{+}$and $g_{\times}$as measured from our red, blue, green, and blue+red galaxy samples (Section 3.4). For all samples, the $\times$ component is consistent with a null detection well within $2 \sigma$ at all radii, indicating the reliability of our distortion analysis. The red and blue populations show a very similar form of the radial $g_{+}$profile that declines smoothly from the cluster center. The observed tangential distortion signal is significant with a total detection $\mathrm{S} / \mathrm{N}$ of 8.1 and 5.1 for the red and the blue sample, respectively, both remaining positive to the limit of our data, $\theta_{\max }=16^{\prime}$. The detection significance is improved to $9.3 \sigma$ using a full composite sample of Subaru blue+red background galaxies (see the top panel of Figure 9).

In Figure 5 we also compare the Subaru data with the results obtained from CFHT/MegaCam data (Section 3.1) using the same analysis pipeline as described in Section 3. For this we identified 15,875 background galaxies $\left(n_{g} \approx 4.5\right.$ galaxies $\operatorname{arcmin}^{-2}$ ) with MegaCam $g r z$ photometry using our CC background selection method (Section 3.4) and estimated a mean depth of $z_{s, \text { eff }} \approx 1.09$, comparable to that of the Subaru full background sample $\left(z_{s, \text { eff }}=1.15 \pm 0.1, n_{g} \approx\right.$ 13 galaxies $\operatorname{arcmin}^{-2}$; Section 3.5). This comparison shows excellent agreement where the data overlap, demonstrating the robustness of our analysis.

\subsubsection{Magnification Bias}

For the number counts to measure magnification, we follow the prescription of Umetsu et al. (2011b). We use a sample of red galaxies (Section 3.4), for which the intrinsic count slope $s$ at faint magnitudes is relatively flat, $s \sim 0.1$, so that

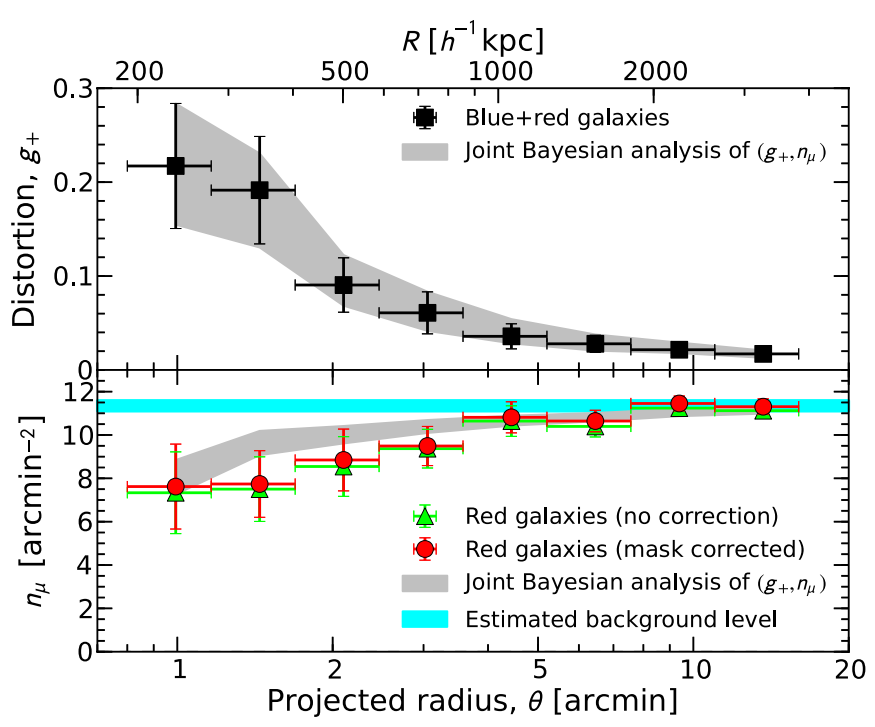

Figure 9. Cluster weak-lensing radial profiles as measured from background galaxies registered in deep Subaru images. The top panel shows the tangential reduced shear profile $g_{+}(\theta)$ (squares) based on Subaru distortion data of the full background (red+blue) sample. The bottom panel shows the count depletion profiles $n(\theta)$ due to magnification for a flux-limited sample of red background galaxies. The circles and triangles show the respective results with and without the mask correction due to bright foreground objects and cluster members. The horizontal bar represents the constraints on the unlensed count normalization, $n_{0}$, as estimated from Subaru data. Also shown in each panel is the joint Bayesian fit $(68 \% \mathrm{CL})$ to both profiles.

(A color version of this figure is available in the online journal.)

a net count depletion results (Broadhurst et al. 2005a; Umetsu \& Broadhurst 2008; Umetsu et al. 2010, 2011b). The blue background population, on the other hand, tends to have a steeper intrinsic count slope close to the lensing invariant slope $(s=0.4)$.

The count-in-cell statistic $N(\boldsymbol{\theta})$ is measured from a fluxlimited sample of red background galaxies on a regular grid of equal-area cells, each with a constant solid angle $\Delta \Omega$. The practical difficulty here is contamination due to the intrinsic clustering of background galaxies, which locally can be larger than the lensing-induced signal in a given cell. In order to obtain a clean measure of the lensing signal, such intrinsic clustering needs to be downweighted and averaged over (e.g., Broadhurst et al. 1995; Umetsu \& Broadhurst 2008).

To overcome this problem, we azimuthally average the red galaxy counts $N(\boldsymbol{\theta})$ and obtain the average surface number density $n_{\mu, i} \equiv n_{\mu}\left(\theta_{i}\right)=\left\langle d N\left(\theta_{i}\right) / d \Omega\right\rangle$ as a function of radius from the cluster center $(i=1,2, \ldots, N)$. Here we use the approach developed in Umetsu et al. (2011b) to account and correct for the masking effect due to bright cluster galaxies, foreground objects, and saturated objects. The errors $\sigma_{\mu, i}$ for $n_{\mu, i}$ include both contributions from Poisson errors in the counts and contamination due to intrinsic clustering of red background galaxies. Thanks to the wide field of view Subaru/SuprimeCam, the normalization and slope parameters for the red sample are reliably estimated as $n_{0}=11.4 \pm 0.3$ galaxies $^{-2}$ and $s=0.133 \pm 0.245$ from the coverage-corrected source counts in the outer region $\left(\gtrsim 10^{\prime}\right)$.

We show in the bottom panel of Figure 9 the resulting magnification profile derived from our flux-limited sample of red background galaxies $\left(z^{\prime}<24.6 \mathrm{mag}\right.$; see Table 3$)$. A strong depletion of the red galaxy counts is seen in the central, highdensity region of the cluster and clearly detected out to $\lesssim 4^{\prime}$ from 
the cluster center. The statistical significance of the detection of the depletion signal is $4.4 \sigma$, which is about half the $\mathrm{S} / \mathrm{N}$ of the tangential distortion derived from the full background sample shown in the top panel of Figure 9. The magnification measurements with and without the masking correction are roughly consistent with each other.

\subsection{Mass Profile Reconstruction}

The relation between observable distortion $(g)$ and underlying convergence $(\kappa)$ is non-local. Hence, the mass distribution derived from distortion data alone suffers from a mass-sheet degeneracy (Section 2).

Here we construct a radial mass profile from complementary lens distortion and magnification measurements, $\left\{g_{+, i}\right\}_{i=1}^{N}$ and $\left\{n_{\mu, i}\right\}_{i=1}^{N}$, following the Bayesian prescription given by Umetsu et al. (2011b), effectively breaking the mass-sheet degeneracy. A brief summary of this Bayesian method is provided in Appendix A.1. The model is described by a vector $s$ of parameters containing the discrete convergence profile $\left\{\kappa_{i}\right\}_{i=1}^{N}$ in the subcritical regime $\left(\theta_{i}>\theta_{\text {Ein }}\right)$ and the average convergence within the inner radial boundary $\theta_{\min }$ of the weak-lensing data, $\bar{\kappa}_{\text {min }} \equiv \bar{\kappa}\left(<\theta_{\min }\right)$, so that $\boldsymbol{s}=\left\{\bar{\kappa}_{\min }, \kappa_{i}\right\}_{i=1}^{N}$, being specified by $(N+1)$ parameters.

We find a consistent mass profile solution $s$ based on a joint Bayesian fit to the observed distortion and magnification measurements, as shown in Figure 9. The detection significance has been improved from $9.3 \sigma$ to $11.4 \sigma$ by adding the magnification measurement, corresponding to an improvement by $\sim 23 \%$, compared to the lensing distortion signal (Umetsu et al. 2011b; Coe et al. 2012).

The resulting mass profile $s$ is shown in Figure 6, along with our primary strong-lensing model (Sections 4.1-4.3). Our independent strong- and weak-lensing mass profiles are in good agreement where they overlap, and together they form a well-defined mass profile. The outer mass profile derived from weak lensing exhibits a fairly shallow radial trend with a nearly isothermal logarithmic density slope in projection, $\gamma_{2 \mathrm{D}} \equiv-d \ln \Sigma / d \ln R \sim 1$. Note that this flat behavior is not clearly evident in the tangential distortion profile, which is insensitive to sheet-like mass overdensities (Section 2). To constrain the cluster properties from the composite halo+LSS mass profile, this LSS contribution needs to be taken into account and corrected for. We will come back to this point in Sections 6.2 and 6.4.

Also shown in Figures 6 and 7 is a purely shear-based reconstruction using the one-dimensional (1D) method of Umetsu \& Broadhurst (2008; see also Umetsu et al. 2010), based on the nonlinear extension of aperture mass densitometry (Clowe et al. 2000). Here we have adopted a zero-density boundary condition in the outermost radial bin, $16^{\prime} \leqslant \theta \leqslant 18^{\prime}$. The total $\mathrm{S} / \mathrm{N}$ in the recovered mass profile is $\approx 9.2$, which agrees well with $\approx 9.3$ in the $g_{+}$profile (Section 5.2.1). Our results with different combinations of lensing measurements and boundary conditions, having different systematics, are in agreement with each other. This consistency demonstrates that our results are robust and insensitive to systematic errors.

The projected cumulative mass profile $M_{2 \mathrm{D}}(<\theta)$ is given by integrating the density profile $s=\left\{\bar{\kappa}_{\min }, \kappa_{i}\right\}_{i=1}^{N}$ (see Appendices $A$ and B of Umetsu et al. 2011b) as

$$
M_{2 \mathrm{D}}\left(<\theta_{i}\right)=\pi\left(D_{l} \theta\right)^{2} \Sigma_{\text {crit }} \bar{\kappa}_{\min }+2 \pi D_{l}^{2} \Sigma_{\text {crit }} \int_{\theta_{\min }}^{\theta_{i}} d \ln \theta \theta^{2} \kappa(\theta)
$$

We compare in Figure 7 the resulting $M_{2 \mathrm{D}}$ profiles derived here from a wide variety of strong- (Section 4) and weak-lensing analyses, along with the model-independent Einstein-radius constraints of $M_{2 \mathrm{D}}\left(<17^{\prime \prime}\right)=5.8_{-1.4}^{+1.3} \times 10^{13} M_{\odot} h^{-1}$ at $\theta_{\text {Ein }}=$ $17^{\prime \prime} \pm 2^{\prime \prime}\left(z_{s}=1.03\right)$ and $M_{2 \mathrm{D}}\left(<28^{\prime \prime}\right)=1.1_{-0.3}^{+0.2} \times 10^{14} M_{\odot} h^{-1}$ at $\theta_{\text {Ein }}=28^{\prime \prime} \pm 3^{\prime \prime}\left(z_{s}=2.54\right) .{ }^{42}$ Again, we find good agreement in the regions of overlap among the results obtained from a variety of lensing analyses, ensuring consistency of our lensing analysis and methods.

Unlike the non-local distortion effect, the magnification falls off sharply with increasing distance from the cluster center. For MACS1206, we find $\kappa \lesssim 1 \%$ at radii $\gtrsim 10^{\prime}$, where the expected level of the depletion signal is $n_{\mu} / n_{0}-1 \approx-2 \kappa$ for a maximally depleted sample with $s=0$, indicating a depletion signal of $\lesssim 2 \%$ in the outer region where we have estimated the unlensed background counts, $n_{0}$. This level of signal is smaller than the fractional uncertainties in estimated unlensed counts $n_{0}$ of $3 \%$ (Section 5.2.2), thus consistent with the assumption. Note that the calibration uncertainties in our observational parameters $\left(n_{0}, s, \omega\right)$ have been marginalized over in our Bayesian analysis (the Appendix).

In the presence of magnification, one probes the number counts at an effectively fainter limiting magnitude: $m_{\text {cut }}+$ $2.5 \log _{10} \mu(\theta)$. The level of magnification is on average small in the weak-lensing regime but reaches $\mu \approx 1.6$ (at $z_{s, \text { eff }} \approx$ 1.1) for the innermost bin in this cluster. Hence, we have implicitly assumed in our analysis that the power-law behavior (Equation (7)) persists down to $\sim 0.5 \mathrm{mag}$ fainter than $m_{\text {cut }}$ where the count slope may be shallower. For a given level of count depletion, an underestimation of the effective count slope could lead to an underestimation of $\mu$, thus biasing the resulting mass profile. However, the count slope for our data flattens only slowly with depth varying from $s \sim 0.13$ to $\sim 0.05$ from a limit of $z^{\prime}=24.6-25.1 \mathrm{mag}$, so that this introduces a small correction of only $\sim 10 \%$ for the most magnified bins $(\mu \sim 2)$. In fact, we have found a good consistency between the results with and without the magnification data.

\section{MASS PROFILE FROM JOINT WEAK- AND STRONG-LENSING ANALYSIS}

In this section, we aim to quantify and characterize the mass distribution of MACS1206 using our comprehensive lensing measurements derived from the deep HST and Subaru observations described in Sections 4 and 5. Here, we compare the cluster lensing profiles with the theoretically and observationally motivated NFW model (Navarro et al. 1997) to characterize the cluster mass profile. Our use of the NFW model enables the most direct comparison with detailed theoretical predictions for the internal structure of DM halos based on $\mathrm{N}$-body simulations (e.g., Duffy et al. 2008; Klypin et al. 2011; Prada et al. 2011; Bhattacharya et al. 2011). The choice of profile shape does not significantly affect the derived halo concentrations (e.g., Duffy et al. 2008).

To be able to constrain the inner density slope, we consider a generalized parameterization of the NFW model (hereafter gNFW) of the form (Zhao 1996; Jing \& Suto 2000)

$$
\rho(r)=\frac{\rho_{s}}{\left(r / r_{s}\right)^{\alpha}\left(1+r / r_{s}\right)^{3-\alpha}},
$$

\footnotetext{
42 Zitrin et al. (2012) quote their full-model-based estimates on the respective integrated masses of $M_{2 \mathrm{D}}=6 \pm 0.7 \times 10^{13} M_{\odot} h^{-1}$ and $M_{2 \mathrm{D}}=0.94 \pm 0.11 \times 10^{14} M_{\odot} h^{-1}$.
} 
where $\rho_{s}$ is the characteristic density, $r_{s}$ is the characteristic scale radius, and $\alpha$ represents the inner slope of the density profile. This reduces to the NFW model for $\alpha=1$. We introduce the radius $r_{-2}$ at which the logarithmic slope of the density is isothermal, i.e., $\gamma_{3 \mathrm{D}}=2$. For the gNFW profile, $r_{-2}=(2-\alpha) r_{s}$, and thus the corresponding concentration parameter reduces to $c_{-2} \equiv r_{\text {vir }} / r_{-2}=c_{\text {vir }} /(2-\alpha)$. We specify the gNFW model with the central cusp slope, $\alpha$, the halo virial mass, $M_{\mathrm{vir}}$, and the concentration, $c_{-2}=c_{\text {vir }} /(2-\alpha)$. We employ the radial dependence of the gNFW lensing profiles given by Keeton (2001).

\subsection{Model-independent Constraints}

First, we constrain the NFW model parameters $\boldsymbol{p} \equiv$ $\left(M_{\mathrm{vir}}, c_{\mathrm{vir}}\right)$ by combining model-independent weak-lensing distortion, magnification, and strong-lensing Einstein-radius measurements, whose systematic errors are well understood from numerical simulations (e.g., Meneghetti et al. 2011; Rasia et al. 2012). The $\chi^{2}$ function for the combined Einstein-radius and weak-lensing constraints is expressed as

$$
\chi^{2}=\chi_{\mathrm{Ein}}^{2}+\chi_{\mathrm{WL}}^{2}
$$

where the $\chi_{\text {Ein }}^{2}$ for the Einstein-radius constraints is defined by (see Umetsu \& Broadhurst 2008; Umetsu et al. 2010)

$$
\chi_{\text {Ein }}^{2}=\sum_{i=1}^{N_{\text {Ein }}} \frac{\left[1-\hat{g}_{+, i}\left(\boldsymbol{p}, z_{s, i}\right)\right]^{2}}{\sigma_{+, i}^{2}},
$$

with $N_{\text {Ein }}$ being the number of independent Einstein-radius constraints $\left\{\theta_{\operatorname{Ein}, i}\right\}_{i=1}^{N_{\text {Ein }}}$ from sources with different redshifts $\left\{z_{s, i}\right\}_{i=1}^{N_{\text {Ein }}}$ and $\hat{g}_{+, i}\left(\boldsymbol{p}, z_{s, i}\right)=\hat{g}\left(\theta_{\operatorname{Ein}, i} \mid \boldsymbol{p}, z_{s, i}\right)$ being the NFW model prediction for the reduced tangential shear at $\theta=$ $\theta_{\text {Ein }, i}$, evaluated at the source redshift $z_{s}=z_{s, i}$. Note that the Einstein radius marks the point of maximum distortion, $g_{+}=(\bar{\kappa}-\kappa) /(1-\kappa)=1$, i.e., $\bar{\kappa}=1$ within $\theta_{\text {Ein. }}$. The $\chi^{2}$ function for our full weak-lensing analysis (Section 5.3) is described by

$$
\chi_{\mathrm{WL}}^{2}=\sum_{i, j}\left[s_{i}-\hat{s}_{i}\left(\boldsymbol{p}, z_{s, \mathrm{eff}}\right)\right]\left(\mathcal{C}_{\mathrm{WL}}\right)_{i j}^{-1}\left[s_{j}-\hat{s}_{j}\left(\boldsymbol{p}, z_{s, \mathrm{eff}}\right)\right]
$$

where $s=\left\{\bar{\kappa}_{\min }, \kappa_{i}\right\}_{i=1}^{N}$ is the mass profile reconstructed from the combined lens distortion and magnification measurements, $\hat{\boldsymbol{s}}\left(\boldsymbol{p}, z_{s, \text { eff }}\right)$ is the NFW model prediction for $\boldsymbol{s}$, and $\mathcal{C}_{\mathrm{WL}}$ is the full covariance matrix of $s$ defined as

$$
\mathcal{C}_{\mathrm{WL}}=\mathcal{C}+\mathcal{C}^{\mathrm{lss}}
$$

with $\mathcal{C}$ being responsible for statistical measurement errors (Appendix A.1) and $\mathcal{C}^{\text {lss }}$ being the cosmic covariance matrix responsible for the effect of uncorrelated LSS along the line of sight (Hoekstra 2003; Hoekstra et al. 2011; Umetsu et al. 2011a; Oguri \& Takada 2011). ${ }^{43}$ In all modeling below, the effective source redshift $z_{s, \text { eff }}=1.15 \pm 0.1$ of our full background sample is treated as a nuisance parameter, and its uncertainty is marginalized over. In order to evaluate $\mathcal{C}^{\text {lss }}$, we assume the

\footnotetext{
43 As discussed in Oguri et al. (2010), for a ground-based weak-lensing analysis, the shot noise is a more dominant source of the measurement errors than the cosmic noise contamination. They found from a weak-lensing analysis of $25 \mathrm{X}$-ray-selected clusters that the best-fit parameters are not largely biased by including the cosmic noise covariance but are in general consistent with each other within statistical uncertainties.
}

concordance $\Lambda \mathrm{CDM}$ cosmological model of Komatsu et al. (2011) and use the fitting formula of Peacock \& Dodds (1996) to compute the nonlinear matter power spectrum. We project the matter spectrum out to an effective source redshift of $z_{s, \text { eff }}=$ 1.15 to calculate $\mathcal{C}^{\text {lss }}$ for weak-lensing observations. For details, see Umetsu et al. (2011a). For Einstein-radius measurements, we conservatively assume an rms displacement of $2^{\prime \prime}$ due to uncorrelated LSS, as predicted by recent theoretical work $\left(\sim 2^{\prime \prime}\right.$ for a distant source at $z_{s} \sim 2.5$; see Host 2012; Jullo et al. 2010). This is combined in quadrature with the measurement error in $\theta_{\text {Ein }}($ Table 1$)$ to estimate a total uncertainty $\sigma_{+, i} \cdot{ }^{44}$

For strong lensing, we use double Einstein-radius constraints $\left(N_{\text {Ein }}=2\right)$ from the multiple-image systems at $z_{s}=1.03$ and $z_{s}=2.54$ (Table 1). For weak lensing, the cluster mass profile $s$ is measured in $N+1=9$ bins. Hence, we have a total of 11 constraints.

The resulting constraints on the NFW model parameters are summarized in Table 5.

\subsubsection{Weak-lensing Constraints}

First of all, when no magnification or strong-lensing information is included, the best-fit model is obtained from a tangential reduced shear fitting as $M_{\text {vir }}=0.99_{-0.26}^{+0.32} \times 10^{15} M_{\odot} h^{-1}$ and $c_{\text {vir }}=5.7_{-2.1}^{+3.6}$ with $\chi_{\min }^{2} /$ dof $=3.3 / 6 .^{45}$

Next, when magnification bias is included to break the masssheet degeneracy, we find $M_{\mathrm{vir}}=1.15_{-0.28}^{+0.34} \times 10^{15} M_{\odot} h^{-1}$ and $c_{\mathrm{vir}}=4.0_{-1.4}^{+2.1}\left(\chi_{\min }^{2} / \mathrm{dof}=4.5 / 7\right)$, which is consistent within the large uncertainties with the purely shear-based results but is in favor of a larger $M_{\text {vir }}$ and a smaller $c_{\text {vir }}$, owing to the shallow outer mass profile. This is demonstrated in the bottom panel of Figure 6, which shows significant deviations $\Delta \kappa$ from our reference NFW model $\left(M_{\mathrm{vir}} \approx 1.1 \times 10^{15} M_{\odot} h^{-1}\right.$ and $c_{\text {vir }} \approx 6.9$; see Section 6.4) at cluster outskirts, $R \gtrsim 1 \mathrm{Mpc} h^{-1}$ $\left(\theta \gtrsim 4^{\prime}\right)$. This large-scale excess in projected mass is also shown in Figure 7 in terms of the integrated projected mass profile $M_{2 \mathrm{D}}(<R)$. Both fits here underestimate the observed Einstein radius (see Table 5).

\subsubsection{Combining Einstein-radius Constraints with Weak Lensing}

When the inner Einstein-radius information is combined with weak lensing, we obtain tighter parameter constraints. By combining all lens distortion, magnification, and Einsteinradius constraints (Equation (16)), we find $M_{\text {vir }}=1.0_{-0.2}^{+0.3} \times$ $10^{15} M_{\odot} h^{-1}$ and $c_{\mathrm{vir}}=6.8_{-1.6}^{+2.1}\left(\chi_{\min }^{2} / \mathrm{dof}=6.9 / 9\right)$, corresponding to an effective Einstein radius of $\theta_{\text {Ein }} \approx 26^{\prime \prime}$ at $z_{s}=2.5$. That is, a slightly higher concentration is favored to reproduce the observed large Einstein radii (Broadhurst \& Barkana 2008).

\subsection{Mass and Galaxy Distribution Shapes in and around the Cluster}

The presence of surrounding LSS in MACS1206 has a nonnegligible impact on the determination of cluster mass profile especially at large radii (Sections 5.3 and 6.1). It is therefore necessary to assess and correct for their effects on the projected mass profile. Here we use two different methods to quantify the

\footnotetext{
44 Following Umetsu \& Broadhurst (2008), we propagate the uncertainty in $\theta_{\text {Ein }}$ to $g_{+}$assuming a singular isothermal sphere (SIS) model. At $r \lesssim r_{s}$, the density slope of NFW is shallower than that of SIS (see Figure 1 of Wright \& Brainerd 2000), so that this gives a fairly conservative estimate of $\sigma_{+}\left(\theta_{\operatorname{Ein}}\right)$. 45 We follow Hoekstra (2003) to calculate the cosmic shear covariance matrix.
} 
Table 5

Best-fit NFW Model Parameters for MACS1206

\begin{tabular}{|c|c|c|c|c|c|c|}
\hline \multirow{2}{*}{$\begin{array}{l}M_{\mathrm{vir}^{\mathrm{a}}} \\
\left(10^{15} M_{\odot} h^{-1}\right)\end{array}$} & \multirow[t]{2}{*}{$c_{\mathrm{vir}}$} & \multirow[t]{2}{*}{$\chi^{2} /$ dof } & \multirow{2}{*}{$\begin{array}{c}\theta_{\text {Ein }}{ }^{b} \\
\left({ }^{\prime \prime}\right)\end{array}$} & \multicolumn{2}{|c|}{$N^{\mathrm{c}}$} & \multirow[t]{2}{*}{ Method } \\
\hline & & & & WL & SL & \\
\hline $0.99_{-0.26}^{+0.32}$ & $5.7_{-2.1}^{+3.6}$ & $3.3 / 6$ & 21 & 8 & 0 & (1) WL tangential distortion (Section 6.1.1) \\
\hline $1.15_{-0.28}^{+0.34}$ & $4.0_{-1.4}^{+2.1}$ & $4.5 / 7$ & 14 & 9 & 0 & (2) WL tangential distortion + magnification (Section 6.1.1) \\
\hline $\begin{array}{l}1.15_{-0.20}^{+0.25} \\
\end{array}$ & $7.5_{-1.8}^{+2.5}$ & $10.6 / 6$ & 32 & 8 & 0 & (3) WL(2) + LSS correction ${ }^{\mathrm{d}}$ (Section 6.4) \\
\hline $0.88_{-0.21}^{+0.25}$ & $8.0_{-1.7}^{+2.3}$ & $3.9 / 8$ & 28 & 8 & 2 & (4) $\mathrm{WL}(1)+$ Einstein radius $^{\mathrm{e}}($ Section 6.1.2) \\
\hline $0.97_{-0.23}^{+0.28}$ & $6.8_{-1.6}^{+2.2}$ & $6.9 / 9$ & 26 & 9 & 2 & (5) $\mathrm{WL}(2)+$ Einstein radius (Section 6.1.2) \\
\hline $1.14_{-0.18}^{+0.22}$ & $6.6_{-0.9}^{+1.0}$ & $24.2 / 31$ & 28 & 8 & 25 & (6) $\mathrm{WL}(2)+\mathrm{SL}^{\mathrm{f}}($ Section 6.5) \\
\hline $1.07_{-0.16}^{+0.20}$ & $6.9_{-0.9}^{+1.0}$ & $18.0 / 31$ & 28 & 8 & 25 & (7) $\mathrm{WL}(3)+\mathrm{SL}=$ our primary NFW result (Section 6.5 ) \\
\hline
\end{tabular}

Notes. All our methods take into account the cosmic covariance from distant, uncorrelated large-scale structure (LSS) projected along the line of sight. For weak lensing, the source redshift uncertainty $\left(z_{s, \text { eff }}=1.15 \pm 0.1\right)$ of our background sample has been marginalized over.

${ }^{\text {a }}$ The virial overdensity is $\Delta_{\text {vir }} \approx 132$ times the critical density of the universe at $z=0.439$ in the adopted cosmology $\left(\Omega_{m}=0.3, \Omega_{\Lambda}=0.7\right)$.

b Effective Einstein radius for a source at $z_{s}=2.5$ predicted by the model. The observed value is $28^{\prime \prime} \pm 3^{\prime \prime}$.

${ }^{\mathrm{c}}$ Respective numbers of weak- and strong-lensing constraints.

${ }^{\mathrm{d}}$ Excluding the elongated LSS around the cluster extending along the NW-SE direction (see Figure 8).

e Combining with double Einstein-radius constraints of $\theta_{\text {Ein }}=17^{\prime \prime} \pm 2^{\prime \prime}$ at $z_{s}=1.03$ and $\theta_{\text {Ein }}=28^{\prime \prime} \pm 3^{\prime \prime}$ at $z_{s}=2.54$. Additionally, an rms displacement of $2^{\prime \prime}$ is assumed for each system due to uncorrelated LSS projected along the line of sight and is combined in quadrature with the respective measurement error to estimate a total uncertainty.

${ }^{\mathrm{f}}$ Combining with the inner strong-lensing-based mass profile derived from an MCMC implementation of Zitrin et al. (2012) (Sections 4.1-4.3). The outer fitting radius is limited to less than $12^{\prime}$ for direct comparison with Method 7 based on the LSS-corrected weak-lensing profile (Section 6.4).

ellipticity and orientation of the projected mass distribution in and around the cluster.

First, following the prescription given by Umetsu et al. (2009), we introduce mass-weighted quadrupole shape moments around the cluster center, in analogy to Equation (8), defined as

$$
Q_{\alpha \beta}=\int_{\Delta \theta \leqslant \theta_{\max }} d^{2} \theta \Delta \theta_{\alpha} \Delta \theta_{\beta} \Sigma(\boldsymbol{\theta}) \quad(\alpha, \beta=1,2),
$$

where $\theta_{\max }$ is the circular aperture radius and $\Delta \theta_{\alpha}$ is the angular displacement vector from the cluster center. We construct with $\left\{Q_{\alpha \beta}\right\}$ a spin-2 ellipticity measure $e_{\Sigma}=\left|e_{\Sigma}\right| e^{2 i \phi_{e}}$, where the ellipticity is defined such that, for an ellipse with major and minor axes $a$ and $b$, it reduces to $\left|e_{\Sigma}\right|=1-b / a$ and $\phi_{e}$ is the position angle of the major axis (Bertin \& Arnouts 1996), measured north of west here. Similarly, the spin-2 ellipticity for the cluster galaxies is defined using the surface number and $R_{\mathrm{c}}$-band luminosity density fields of CC-selected cluster galaxies (Section 5.1), $\Sigma_{n}(\boldsymbol{\theta})$ and $\Sigma_{l}(\boldsymbol{\theta})$. We calculate weighted moments using only those pixels above the $2 \sigma$ threshold with respect to the background level (estimated with the biweight scale and location; see Beers et al. 1990). Practical shape measurements are done using pixelized maps shown in Figure 8.

Next, we constrain the ellipticity and orientation of the projected mass distribution by directly fitting a 2D shear map with a single elliptical lens model. Here, we closely follow the prescription given by Oguri et al. (2010) to construct an elliptical NFW (eNFW, hereafter) model (see also Oguri et al. 2012), by introducing the mass ellipticity $\left|e_{\Sigma}\right|=1-b / a$ in the isodensity contours of the projected NFW profile $\Sigma(R)$ as $R^{2} \rightarrow$ $X^{2}\left(1-\left|e_{\Sigma}\right|\right)+Y^{2} /\left(1-\left|e_{\Sigma}\right|\right)$ (for details, see Oguri et al. 2010). ${ }^{46}$ The model shear field is computed by solving the 2D Poisson equation (Keeton 2001). We then construct from Subaru data a lens distortion map $\left(g_{1}(\boldsymbol{\theta}), g_{2}(\boldsymbol{\theta})\right)$ and its covariance matrix $\mathcal{C}_{g}$

\footnotetext{
46 As noted by Oguri et al. (2010), this elliptical model includes a triaxial halo model (Jing \& Suto 2000), which gives a better description of CDM halos in $\mathrm{N}$-body simulations than the spherical model.
}

Table 6

Ellipticity and Position Angle Measurements

\begin{tabular}{lccc}
\hline \hline Method & $\begin{array}{c}\theta_{\max }{ }^{\mathrm{a}} \\
\left(^{\prime}\right)\end{array}$ & Ellipticity $^{\mathrm{b}}$ & $\begin{array}{c}\mathrm{PA}^{\mathrm{c}} \\
(\mathrm{deg})\end{array}$ \\
\hline BCG & $10^{\prime \prime}$ & $0.53_{-0.03}^{+0.03}$ & $15.0_{-2.3}^{+2.3}$ \\
Chandra X-ray & $1{ }^{\prime}$ & $0.30_{-0.03}^{+0.03}$ & $21.9_{-1.7}^{+1.7}$ \\
Galaxy density & $8^{\prime}$ & $0.53_{-0.04}^{+0.04}$ & $15.7_{-5.9}^{+1.3}$ \\
Galaxy light & $8^{\prime}$ & $0.41_{-0.06}^{+0.06}$ & $19.0_{-5.4}^{+5.9}$ \\
WL mass map & $8^{\prime}$ & $0.37_{-0.13}^{+0.13}$ & $19.4_{-17.7}^{+8.5}$ \\
WL 2D shear fit & $8^{\prime}$ & $0.68_{-0.28}^{+0.18}$ & $28.6_{-7.9}^{+5.8}$ \\
\hline
\end{tabular}

Notes.

${ }^{\text {a }}$ Circular aperture radius.

${ }^{\mathrm{b}}$ Ellipticity modulus defined such that, for an ellipse with major and minor axes $a$ and $b$, it reduces to $1-b / a$.

${ }^{c}$ Position angle of the major axis measured north of west.

(Equation (A5)) on a 2D Cartesian grid with 1' spacing, centered at the BCG. We exclude from our analysis the five innermost cells lying in the central region, $\theta<1^{\prime}$, to avoid systematic errors (see Appendix A.2). The halo centroid is fixed to the BCG position. Accordingly, the eNFW model is specified by four model parameters, $\boldsymbol{p}=\left(M_{\mathrm{vir}}, c_{\mathrm{vir}},\left|e_{\Sigma}\right|, \phi_{e}\right)$. The constraints on individual parameters are obtained by projecting the $2 \mathrm{D}$ shear likelihood function (Equation (A6) in Appendix A.2) to the parameter space (or, minimizing $\chi^{2}$ ).

In Table 6, we summarize our cluster ellipticity and orientation measurements. In this analysis, we are mainly interested in the orientation of the ellipticity, in order to correct for the effects of LSS along the axis of elongation. An overall agreement is found between the shapes of mass, light, and galaxy distributions in MACS1206, especially in terms of orientation (Figure 8), within large uncertainties (Table 6). The mass distribution in and around the cluster is aligned well with the luminous galaxies in the green sample, composed mostly of cluster member galaxies (Section 3.4). For all cases, the position angle $\phi_{e}$ 


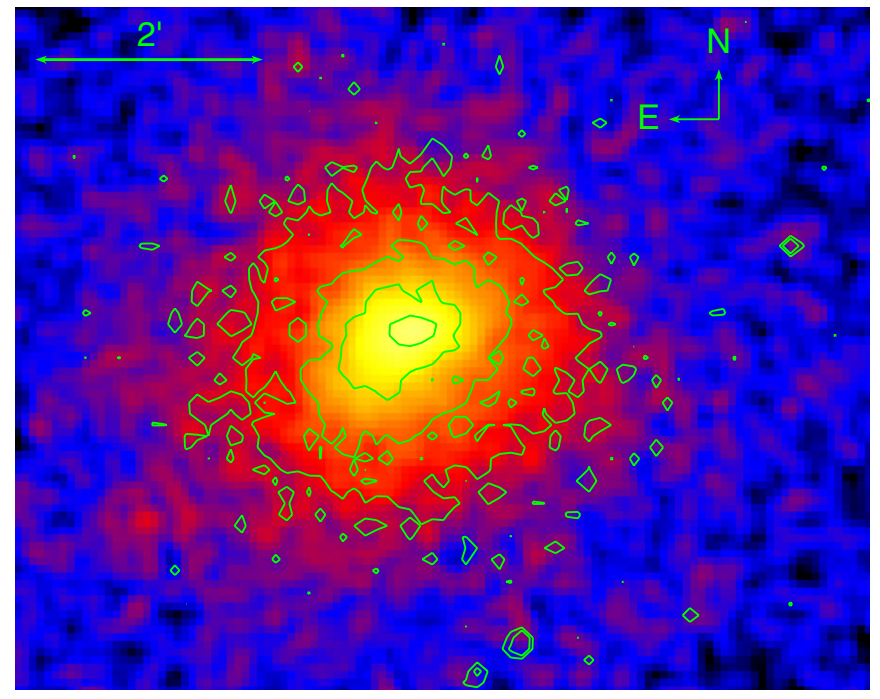

Figure 10. Logarithmically scaled XMM-Newton mosaic, exposure-corrected image of MACS1206 in the $0.5-2 \mathrm{keV}$ band, smoothed with a Gaussian of 8".0 FWHM. Overlaid are contours from the exposure-corrected Chandra $0.5-2 \mathrm{keV}$ image, smoothed with a Gaussian of 1'.5 FWHM. The field size is $7.5 \times 66^{\prime} .0$, with north to the top and east to the left. The scale bar shows $2^{\prime}$ or about $680 \mathrm{kpc} \approx 1.1 r_{2500} . \mathrm{X}$-ray emission is concentrated around and peaked on the BCG but shows some elongation within $\theta \lesssim 1^{\prime}$ at a position angle around $120^{\circ}$ east of north, aligned with the orientation of the projected mass distribution. At larger distances from the cluster center, the cluster appears fairly round in both Chandra and XMM images.

(A color version of this figure is available in the online journal.)

of the major axis is found to be fairly constant with radius $\theta_{\max }$ and lies in the range $15^{\circ} \lesssim \phi_{e} \lesssim 30^{\circ}$.

In the central region, we find a projected mass ellipticity of $\left|e_{\Sigma}\right| \sim 0.3$ and a position angle of $\phi_{e} \sim 14^{\circ}$ from the PIXELENS analysis; we obtain consistent values for both $\left|e_{\Sigma}\right|$ and $\phi_{e}$ from a different strong-lensing analysis (C. Grillo et al. 2012, in preparation) using only System 7 of Zitrin et al. (2012). A similar value is found for the projected mass ellipticity of $\left|e_{\Sigma}\right|=0.26 \pm 0.16\left(\phi_{e} \sim 19^{\circ}\right)$ at $\theta_{\max }=4^{\prime}$ using the weaklensing $\Sigma$ map. From an elliptical King model fit to Chandra X-ray data (Figure 10; for details, see Section 7.4), we find an ellipticity of $0.30 \pm 0.03(a / b \approx 1.5)$ and $\phi_{e}=21.9 \pm 1.7$ at $\theta_{\max }=1.5$.

On the other hand, we obtained higher values of ellipticity on large angular scales beyond the cluster virial radius, $\theta_{\text {vir }} \equiv$ $r_{\text {vir }} / D_{l} \sim 7^{\prime}$. We find $\left|e_{\Sigma}\right| \sim 0.4-0.5$ at $\theta_{\max }=8^{\prime}$ using the pixelized cluster mass, galaxy, and light distributions. From the 2D shear fitting to a single eNFW model, the projected mass ellipticity is constrained in the range $\left|e_{\Sigma}\right|=0.68_{-0.28}^{+0.18}\left(\left|e_{\Sigma}\right| \gtrsim\right.$ 0.4 or $a / b \gtrsim 1.7$ at $1 \sigma)$ at $\theta_{\max }=8^{\prime}$. This apparent increase in ellipticity with radius could be partly explained by the additional contribution from the surrounding LSS that is extended along the cluster major axis. Note that the observed tendency for the shear-based method to yield higher ellipticity estimates, compared to the mass-map-based method, could be due to the non-local nature of the shear field, in conjunction with our single-component assumption in the 2D shear fitting analysis. Overall, this level of ellipticity is consistent within large errors with the mean cluster ellipticity $\left\langle\left|e_{\Sigma}\right|\right\rangle=0.46 \pm 0.04$ obtained by Oguri et al. (2010) from a 2D weak-lensing analysis of 25 $\mathrm{X}$-ray-luminous clusters.

In what follows, we fix the position angle of the NW-SE cluster-LSS major axis to a reference value of $\phi_{e}=20^{\circ}$, which is close to the values derived from the Chandra X-ray data, $\Sigma_{l}$ and $\Sigma$ maps. We note that, in principle, the X-ray structure in a triaxial system is expected to be tilted with respect to the total matter in projection, even in the absence of intrinsic misalignments (see Romanowsky \& Kochanek 1998). In the present work, we define the NW and SE excess regions, respectively, as NW and SE outer cone regions with $\theta>4^{\prime}$ centered on the cluster center, with opening angle $90^{\circ}$ and position angle $\phi_{e}=20^{\circ}$, as defined by the pair of gray solid lines in each panel of Figure 8.

\subsection{BCG-Cluster Alignment}

We have also obtained CLASH constraints on the mean BCG ellipticity and position angle derived from the ACS F814W image. For this we performed a detailed structural analysis on the BCG using the snuc task in the XVISTA software package. ${ }^{47}$ In Figure 11 we show the ACS F814W image, bestfit model, and image residuals after subtraction of the model. No systematic deviations are seen in the residuals between the data and the model, suggesting that the BCG has not undergone any major merger recently. The radial profiles of ellipticity and position angle were measured in several independent radial bins $\left(0^{\prime} .2 \lesssim \theta \lesssim 10^{\prime \prime}\right)$, and their respective (sensitivity weighted) mean values were obtained as $\left\langle e_{\Sigma}\right\rangle=0.53 \pm 0.03$ and $\phi_{e}=$ $(15.0 \pm 2.3) \mathrm{deg}$ (Table 6). Consistent results were found in several other HST bands (ACS F475W to F814W and WFC3 $\mathrm{F} 105 \mathrm{~W}$ to $\mathrm{F} 160 \mathrm{~W}$ ). The mean BCG ellipticity is found to lie in the range $0.46-0.53$ with a small scatter of 0.02 across the ACS and WFC3 bands. The BCG position angle is constrained to be $\phi_{e}=(15.2 \pm 0.4) \mathrm{deg}$, which is in excellent agreement especially with that derived independently from the large-scale distribution $\Sigma_{n}$ of galaxies.

\subsection{Effects of Surrounding Large-scale Structure}

In this subsection, we look into the azimuthal dependence of the radial projected mass distribution, $\Sigma(R, \phi)$, to assess and correct for the effect of surrounding LSS on the cluster mass profile measurement. Because of the non-local nature and inherent insensitivity to sheet-like overdensities of the shear field, it is essential to use the combination of lens magnification and distortion to reconstruct the projected cluster mass distribution embedded in LSS. For this purpose, we extend the 1D Bayesian method of Umetsu et al. (2011b) into a 2D mass distribution by combining the 2D shear pattern $g(\boldsymbol{\theta})$ with the azimuthally averaged magnification measurements $n_{\mu}(\theta)$. In the $2 \mathrm{D}$ analysis, our model $s$ is a vector of parameters containing a set of discrete mass elements on a grid of $N_{\text {cell }}$ independent cells, $\boldsymbol{s}=\left\{\kappa_{m}\right\}_{m=1}^{N_{\text {cell }}}$. A brief summary of this $2 \mathrm{D}$ method is given in Appendix A.2. The details of the method will be presented in our forthcoming paper (K. Umetsu et al. 2012, in preparation).

By combining Subaru distortion and magnification data, we construct here a mass map over a $30 \times 30$ grid with 0.8 spacing, covering a $24^{\prime} \times 24^{\prime}$ field around the cluster $\left(N_{\text {cell }}=900\right)$. We have $2 \times 896$ distortion constraints $\left\{g_{1}\left(\boldsymbol{\theta}_{m}\right)\right\}_{m=1}^{N_{\text {cell }}}$ and $\left\{g_{2}\left(\boldsymbol{\theta}_{m}\right)\right\}_{m=1}^{N_{\text {cell }}}$ over the mass grid, excluding the four innermost cells lying in the cluster central region (see Appendix A.2), and $N=8$ radial magnification constraints $\left\{n_{\mu}\left(\theta_{i}\right)\right\}_{i=1}^{N}$. Hence, we have a total of 1800 constraints (900 dof). Additionally, we marginalize over the calibration uncertainties in the observational parameters $\left(n_{0}, s, \omega\right.$; Section 3.5). The best solution $s$ has been obtained with $\chi_{\min }^{2} /$ dof $=1058 / 900$. We then follow Umetsu \&

\footnotetext{
$\overline{47}$ http://astronomy.nmsu.edu/holtz/xvista/index.html
} 

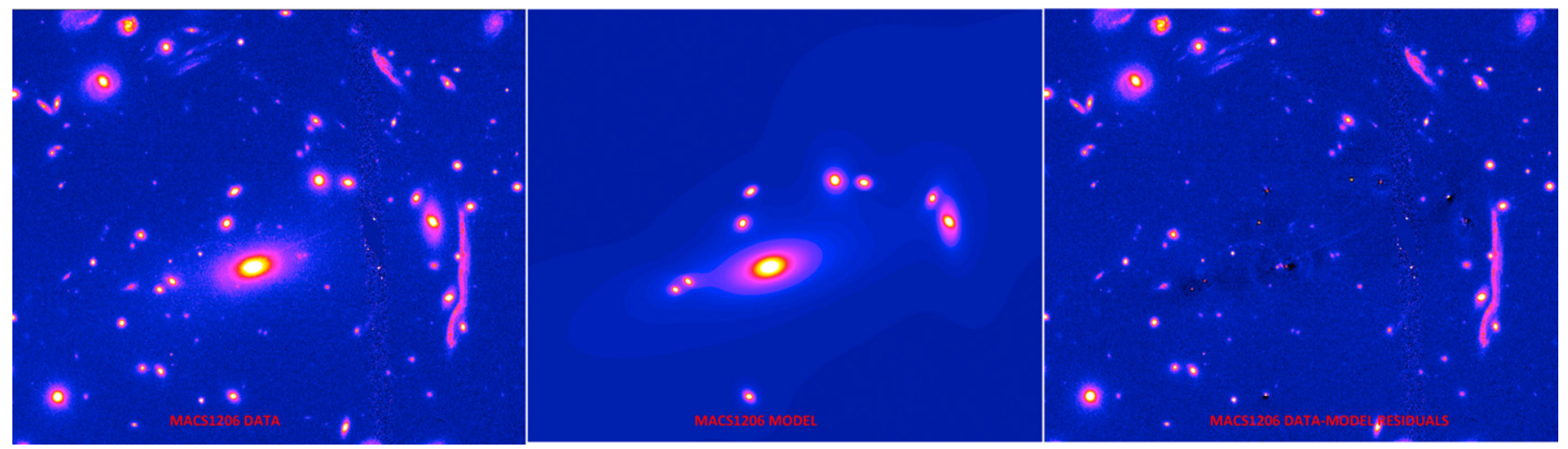

Figure 11. Detailed model fits to the BCG and its nine nearby galaxies in the ACS F814 image $\left(\approx 50^{\prime \prime} \times 45^{\prime \prime}\right)$. The panels show the ACS image (left), best-fit model (middle), and image residuals (right) after subtraction of the model. No systematic deviations are seen in the residuals between the data and the model, suggesting that the BCG has not undergone any major merger recently. North to the top, east to the left.

(A color version of this figure is available in the online journal.)

Broadhurst (2008) to calculate the radial mass distribution $\langle\Sigma(R)\rangle$ and its covariance matrix from a weighted projection of the $\kappa$ map, where we conservatively limit our 2D analysis to radii smaller than $\theta=12^{\prime}\left(R \approx 2.9 \mathrm{Mpc} h^{-1}\right)$. We check that the azimuthally averaged radial mass profile constructed from the $\kappa$ map reproduces our corresponding $1 \mathrm{D}$ results (Section 5.3).

We show in Figure 6 the radial mass distribution obtained excluding the NW and SE excess regions (defined in Section 6.4; see also Figure 8). This weak-lensing mass profile, corrected for the effect of surrounding LSS, exhibits a steeper radial trend than that averaged over all azimuthal angles. We note that a slight remaining excess is seen at $\theta \gtrsim 5^{\prime}\left(R \gtrsim 1.2 \mathrm{Mpc} h^{-1}\right)$. By fitting the "LSS-corrected" mass profile with an NFW profile, we find a higher concentration $c_{\mathrm{vir}}=7.5_{-1.8}^{+2.5}$ with $M_{\mathrm{vir}}=1.15_{-0.20}^{+0.25} \times 10^{15} M_{\odot} h^{-1}\left(\chi_{\min }^{2} / \mathrm{dof}=10.6 / 6\right)$. This model predicts an Einstein radius of $\theta_{\text {Ein }} \approx 32^{\prime \prime}$ for $z_{s}=2.5$, comparable to the observed value, $\theta_{\text {Ein }}=28^{\prime \prime} \pm 3^{\prime \prime}$.

\subsection{Full-lensing Constraints}

As shown in Figures 6 and 7, our weak- and strong-lensing data agree well in their region of overlap. Here we further improve the statistical constraints on the halo parameters $\boldsymbol{p}=$ $\left(M_{\mathrm{vir}}, c_{\mathrm{vir}}, \alpha\right)$ by combining the joint weak-lensing distortion and magnification constraints $\chi_{\mathrm{WL}}^{2}\left(\boldsymbol{p}, z_{s, \text { eff }}\right)$ (Section 6.1) with the inner mass profile $\kappa_{i}$ based on the detailed strong-lensing analysis of Zitrin et al. (2012).

We write the combined $\chi^{2}$ function of our full-lensing constraints as

$$
\chi^{2}=\chi_{\mathrm{WL}}^{2}+\chi_{\mathrm{SL}}^{2}
$$

with $\chi_{\mathrm{SL}}^{2}$ for strong lensing being defined as

$$
\chi_{\mathrm{SL}}^{2}=\sum_{i, j}\left[\kappa_{i}-\hat{\kappa}_{i}(\boldsymbol{p})\right]\left(\mathcal{C}_{\mathrm{SL}}\right)_{i j}^{-1}\left[\kappa_{j}-\hat{\kappa}_{j}(\boldsymbol{p})\right],
$$

where $\kappa_{i}$ is defined in 25 discrete bins over the radial range $\left[4^{\prime \prime}, 53^{\prime \prime}\right]$ (see Section 4) and scaled to a fiducial depth $z_{s}=2.54$ of the strong-lensing observations, matched to the spectroscopically confirmed five-image system (System 4 of Zitrin et al. 2012); $\hat{\kappa}_{i}$ is the theoretical prediction for $\kappa_{i}$; and $\mathcal{C}_{\mathrm{SL}}=\mathcal{C}+\mathcal{C}_{\text {lss }}$ is the bin-to-bin covariance matrix for the discrete $\kappa$ profile, with $\mathcal{C}$ being the self-calibrated covariance matrix derived in Section 4.3 and $\mathcal{C}_{\text {lss }}$ being the cosmic noise contribution. We use a consistent single source plane at $z_{s}=2.54$ to evaluate $\mathcal{C}^{\text {lss }}$.
Table 7

Best-fit Generalized-NFW Model Parameters for MACS1206

\begin{tabular}{lccccc}
\hline \hline Method $^{\mathrm{a}}$ & $\begin{array}{c}M_{\text {vir }} \\
\left(10^{15} M_{\odot} h^{-1}\right)\end{array}$ & $c_{-2}{ }^{\mathrm{b}}$ & $\alpha^{\mathrm{c}}$ & $\chi^{2} /$ dof & $\begin{array}{c}\theta_{\text {Ein }^{\mathrm{d}}} \\
\left.{ }^{\prime \prime}\right)\end{array}$ \\
\hline 6 & $1.17_{-0.22}^{+0.29}$ & $6.3_{-1.5}^{+1.5}$ & $1.09_{-0.42}^{+0.28}$ & $24.1 / 30$ & 28 \\
7 & $1.06_{-0.18}^{+0.23}$ & $7.0_{-1.4}^{+1.5}$ & $0.96_{-0.49}^{+0.31}$ & $18.0 / 30$ & 28 \\
\hline
\end{tabular}

Notes. See for details Section 6.5.

a Fitting method in Table 5.

${ }^{\mathrm{b}}$ Effective concentration parameter for $\mathrm{gNFW}, c_{-2} \equiv r_{\mathrm{vir}} / r_{-2}=c_{\mathrm{vir}} /(2-\alpha)$.

c Central cusp slope of gNFW.

${ }^{\mathrm{d}}$ Effective Einstein radius for a source at $z_{s}=2.5$ predicted by the model. The observed value is $28^{\prime \prime} \pm 3^{\prime \prime}$.

The resulting NFW and gNFW fits are summarized in Tables 5 and 7 , respectively. For both models, we show the respective fits derived with and without the LSS correction for the outer weaklensing profile $\left(R \gtrsim 1 \mathrm{Mpc} h^{-1}\right)$. We find that, when the detailed strong-lensing information is combined with weak lensing, the LSS correction does not significantly affect the fitting results with the adopted NFW/gNFW form. Moreover, all these models properly reproduce the observed location of the Einstein radius, $\theta_{\text {Ein }} \approx 28^{\prime \prime}$.

Here we summarize our primary results obtained with the LSS correction. The confidence contours on the NFW parameters $\left(M_{\mathrm{vir}}, c_{\mathrm{vir}}\right)$ are shown in Figure 12. The constraints are strongly degenerate when only the inner or outer mass profile is included in this fit. Combining complementary weakand strong-lensing information significantly narrows down the statistical uncertainties on the NFW model parameters, placing tighter constraints on the entire mass profile (Model 7 of Table 5): $M_{\text {vir }}=1.07_{-0.16}^{+0.20} \times 10^{15} M_{\odot} h^{-1}$ and $c_{\text {vir }}=6.9_{-0.9}^{+1.0}$ with $\chi_{\min }^{2} /$ dof $=18.0 / 31$, corresponding to a $Q$-value goodness of fit of $Q=0.970$. Next, when $\alpha$ is allowed to vary (Table 7), we find $M_{\text {vir }}=1.06_{-0.18}^{+0.23} \times 10^{15} M_{\odot} h^{-1}, c_{-2}=7.0_{-1.4}^{+1.5}$, and $\alpha=0.97_{-0.23}^{+0.28}$ with $\chi_{\min }^{2} /$ dof $=18.0 / 30$ and $Q=0.960$, being consistent with the simple NFW form with $\alpha=1$. Thus, the addition of the $\alpha$ parameter has little effect on the fit, as shown by the quoted $\chi^{2}$ and $Q$ values. The two-dimensional marginalized constraints on $\left(M_{\mathrm{vir}}, \alpha\right)$ and $\left(c_{-2}, \alpha\right)$ are shown in Figure 13.

\subsection{Impact of the Choice of Strong-lensing Models}

In this subsection, we address the impact of the choice of strong-lensing models on the determination of the halo mass 


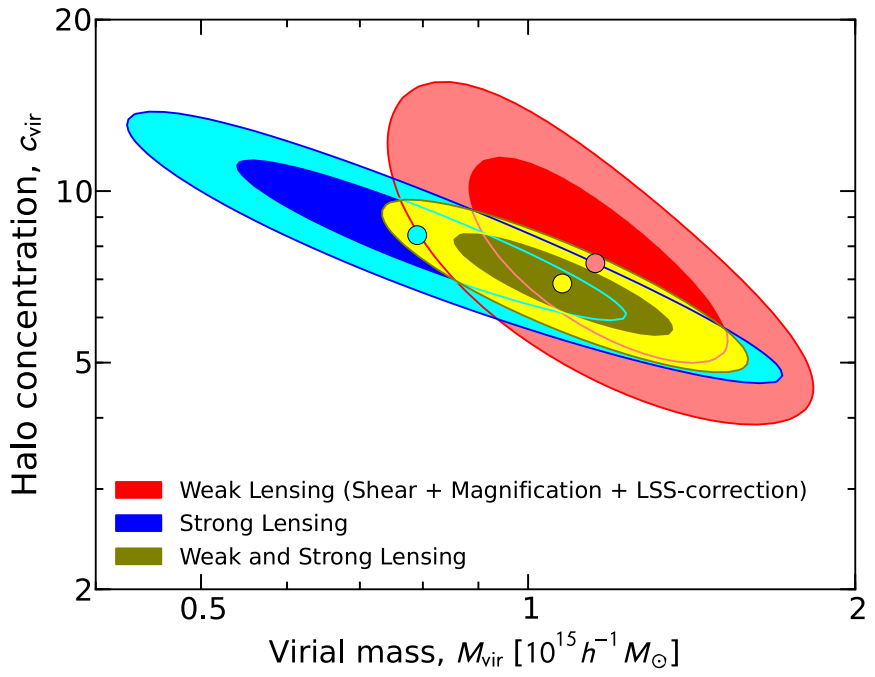

Figure 12. Constraints on the NFW model parameters $\left(M_{\mathrm{vir}}, c_{\mathrm{vir}}\right)$, the halo virial mass and concentration, derived from weak-lensing (red), strong-lensing (blue), and joint weak- and strong-lensing (yellow) analyses. The weak-lensing results are obtained with the LSS correction (Section 6.4). The contours show the $68.3 \%$ and $95.4 \%$ confidence levels, estimated from $\Delta \chi^{2} \equiv \chi^{2}-\chi_{\min }^{2}=2.3$ and 6.17, respectively. The circles indicate the respective best-fit model parameters. For weak lensing, the source redshift uncertainty, $z_{s}=1.15 \pm 0.1$, is marginalized over.

(A color version of this figure is available in the online journal.)

and concentration parameters in a joint weak- and stronglensing analysis. As an alternative choice to the Zitrin et al. (2012) model, we consider here PIXELENs (non-parametric) and LENSTOOL (parametric) models, in combination with our LSS-corrected weak-lensing mass model (Section 6.4). For each case, we define the $\chi^{2}$ function for strong lensing as in Equation (22) and minimize the total $\chi^{2}$ function (Equation (21)) to estimate the NFW parameters $\left(M_{\mathrm{vir}}, c_{\mathrm{vir}}\right)$.

The resulting model constraints are tabulated in Table 8. We find that both parameters based on different strong-lensing profiles are consistent with each other within the statistical errors. This also indicates consistency between these stronglensing models and our weak-lensing measurements, as shown in Figure 7. We find a tendency for PIXELENS to yield somewhat higher mass estimates compared to other strong-lens modeling methods, as discussed by Grillo et al. (2010, their Appendix).

When the NFW (gNFW) form is assumed, the Zitrin et al. (2012) model predicts a somewhat higher concentration and a lower mass than other models as implied by its correspondingly higher central density at $\lesssim 0^{\prime} .2$ (see Figure 7). When the inner fitting radius is increased from $4^{\prime \prime}$ to $12^{\prime \prime}\left(\sim 50 \mathrm{kpc} h^{-1}\right)$, we find a fractional increase of $\sim 9 \%$ in $M_{\text {vir }}$ and a fractional decrease of $\sim 17 \%$ in $c_{\text {vir }}\left(6.9_{-0.9}^{+1.0} \rightarrow 5.7_{-1.1}^{+1.4}\right)$. Including these variations as systematic uncertainties in our mass-concentration determination, the spherical NFW model for MACS1206 is constrained as $M_{\mathrm{vir}}=\left(1.07_{-0.16}^{+0.20} \pm 0.10\right) \times 10^{15} M_{\odot} h^{-1}$ and $c_{\text {vir }}=6.9_{-0.9}^{+1.0} \pm 1.2$ (statistical followed by systematic uncertainty). Similarly, when the central $50 \mathrm{kpc} h^{-1}$ region is excluded from the fit, we have $M_{\text {vir }}=\left(1.17_{-0.20}^{+0.25} \pm 0.10\right) \times 10^{15} M_{\odot} h^{-1}$ and $c_{\text {vir }}=5.7_{-1.1}^{+1.4} \pm 1.2$.

\subsection{Alternative Mass Profile Fits}

Motivated by the apparently shallow projected density profile in the outer regions (cf. XMMU J2235.3-2557 at $z=1.4$; Jee et al. 2009), we consider here a softened power-law sphere

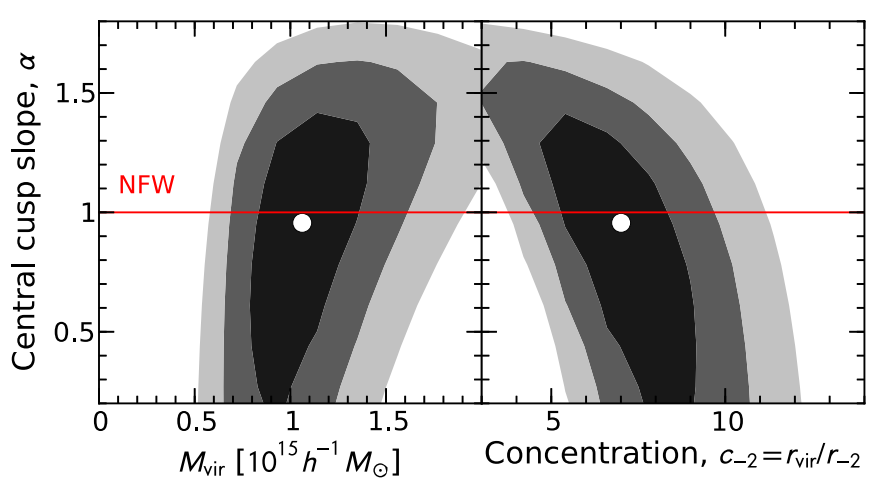

Figure 13. Constraints on the gNFW model parameters, namely, the central cusp slope $\alpha$, the halo virial mass $M_{\mathrm{vir}}$, and the halo concentration $c_{-2}=r_{\mathrm{vir}} / r_{-2}=$ $c_{\mathrm{vir}} /(2-\alpha)$, when all of them are allowed to vary, derived from combined weak and strong lensing. The weak-lensing results are obtained with the LSS correction (Section 6.4). The left and right panels show the two-dimensional marginalized constraints on $\left(M_{\mathrm{vir}}, \alpha\right)$ and $\left(c_{-2}, \alpha\right)$, respectively. In each panel of the figure, the contours show the $68.3 \%, 95.4 \%$, and $99.7 \%$ confidence levels, and the circle indicates the best-fit model parameters.

(A color version of this figure is available in the online journal.)

Table 8

Impact of the Choice of Strong-lensing Models in the Full Lensing Analysis

\begin{tabular}{lcccc}
\hline \hline $\begin{array}{l}M_{\text {vir }} \\
\left(10^{15} M_{\odot} h^{-1}\right)\end{array}$ & $c_{\text {vir }}$ & $\chi^{2} /$ dof & $\begin{array}{c}\theta_{\text {Ein }}{ }^{\mathrm{a}} \\
\left({ }^{\prime \prime}\right)\end{array}$ & Method $^{\mathrm{b}}$ \\
\hline $1.07_{-0.16}^{+0.20}$ & $6.9_{-0.9}^{+1.0}$ & $18.0 / 31$ & 28 & $\mathrm{WL}(3)+$ Zitrin+12 \\
$1.17_{-0.20}^{+0.25}$ & $5.7_{-1.1}^{+1.4}$ & $16.0 / 26$ & 25 & $\mathrm{WL}(3)+$ Zitrin+12 $+50 \mathrm{kpc} h^{-1}$ cut $^{\mathrm{d}}$ \\
$1.37_{-0.22}^{+0.26}$ & $5.8_{-0.8}^{+0.9}$ & $15.4 / 20$ & 29 & $\mathrm{WL}(3)+$ PIXELENS \\
$1.26_{-0.17}^{+0.20}$ & $6.0_{-0.8}^{+0.9}$ & $11.6 / 31$ & 28 & $\mathrm{WL}(3)+$ LeNSTOOL \\
\hline
\end{tabular}

Notes. See for details Section 6.6.

${ }^{a}$ Effective Einstein radius for a source at $z_{s}=2.5$ predicted by the model.

b Combination of strong- and weak-lensing mass models used for the fitting. For all cases, Method 3 of Table 5 is used for weak lensing.

c This corresponds to our best model (Model 7) of Table 5.

d Now applying a central $12^{\prime \prime}\left(\sim 50 \mathrm{kpc} h^{-1}\right)$ cut to the Zitrin et al. (2012) based strong-lensing model.

(SPLS) model (Grogin \& Narayan 1996) as an alternative to the NFW profile and perform profile fitting analyses on our full-range mass profile data (derived from Methods 6 and 7 in Table 5; see Sections 6.4 and 6.5).

The SPLS model has the same number of free parameters as gNFW, namely, three. The SPLS density profile is given by $\rho(r)=\rho_{0}\left(1+r^{2} / r_{c}^{2}\right)^{(\eta-3) / 2}$, where $\rho_{0}=\rho(0)$ is the central density, $r_{c}$ is the core radius, and the power-law index $\eta$ is restricted to lie in the range $0 \leqslant \eta \leqslant 2$ (Grogin \& Narayan 1996). At $r \gg r_{c}, M(<r) \propto r^{\eta}$. This reduces to a non-singular isothermal sphere (NIS) model when $\eta=1$. The fitting results with and without the outer LSS correction (Methods 7 and 6, respectively) are summarized in Table 9.

First, when $\eta$ is fixed to unity (NIS), the NIS model provides acceptable fits, but with larger residuals $\left(\chi^{2}\right)$ compared to the corresponding NFW fits with the same degrees of freedom (31): $\Delta \chi^{2}=\chi_{\min , \mathrm{NIS}}^{2}-\chi_{\min , \mathrm{NFW}}^{2}=2.3($ Method 6$)$ and $5.9($ Method 7$)$ between the best-fit NIS and NFW models. Note that because of the asymptotic $M(<r) \propto r$ behavior, the assumed NIS form leads to substantially higher masses at large radius $\left(r \gg r_{c}\right)$ than what the NFW model predicts $(\sim 35 \%$ higher than the NFW values at $r=1.6 \mathrm{Mpc} h^{-1}$ ).

Next, when the outer slope is allowed to vary, the fit is noticeably improved for the results with the outer LSS correction 
Table 9

Best-fit SPLS Model Parameters for MACS1206

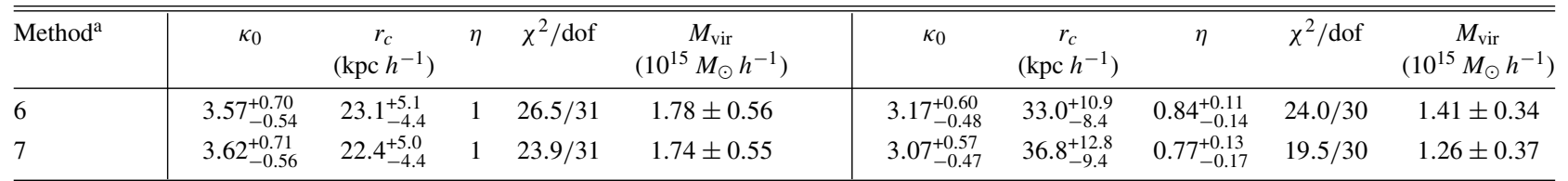

Notes. The convergence profile of the softened power-law sphere (SPLS) model, $\rho(r)=\rho_{0}\left(1+r^{2} / r_{c}^{2}\right)^{(\eta-3) / 2}$, is given by $\kappa(\theta)=\kappa_{0}\left(1+\theta^{2} / \theta_{c}^{2}\right)^{(\eta-2) / 2}$, where $\theta_{c}=r_{c} / D_{l}$ and $\kappa_{0}=B(1 / 2,1-(\eta / 2)) \rho_{0} r_{c} / \Sigma_{\text {crit }}$, with $B$ being the standard Euler beta function. Here $\Sigma_{\text {crit }}$ is evaluated for a source at a reference redshift of $z_{s}=2.54$. For details, see Section 6.7 .

${ }^{a}$ Fitting method in Table 5.

(Method 7), corresponding to a difference of $\Delta \chi^{2}=\chi_{\min , \mathrm{NIS}}^{2}$ $\chi_{\min , \text { SPLS }}^{2}=4.4$ between NIS and SPLS for 1 additional dof. For this, the best-fitting slope parameter is obtained as $\eta=0.77_{-0.17}^{+0.13}\left(\chi_{\min }^{2}=19.5\right.$ for $\left.30 \mathrm{dof}\right)$, corresponding to $2.1 \leqslant \gamma_{3 \mathrm{D}}\left(r \gg r_{c}\right) \leqslant 2.4$. This SPLS model yields a virial mass of $M_{\mathrm{vir}}=(1.26 \pm 0.37) \times 10^{15} M_{\odot} h^{-1}\left(r_{\mathrm{vir}} \approx 1.73 \mathrm{Mpc} h^{-1}\right)$.

\section{DISCUSSION}

\subsection{Lensing Systematics}

Gravitational lensing probes the total mass projected onto the sky along the line of sight, so that the lensing-based cluster mass measurements are sensitive to projection effects arising from (1) additional mass overdensities (underdensities) along the line of sight (Meneghetti et al. 2010b; Rasia et al. 2012) and (2) halo triaxiality (Hennawi et al. 2007; Oguri \& Blandford 2009; Meneghetti et al. 2010b; Rasia et al. 2012).

\subsubsection{Projection of Additional Mass Structures}

The first type of projection effects includes the cosmic noise from distant uncorrelated LSS projected along the same line of sight (Hoekstra 2003) and massive structures within/around the cluster (i.e., cluster substructures and surrounding large-scale filamentary structure).

The former can not only increase statistical uncertainties but also produce covariance between radial bins. Accordingly, this could bias the estimates of cluster parameters. Our methods take into account the estimated contribution of cosmic covariance $\mathcal{C}_{\text {lss }}$ in both weak- and strong-lensing profiles and allow us to properly weight the weak and strong lensing when performing a combined halo fit. In our analysis, we find that the contribution of $\mathcal{C}_{\text {lss }}$ to the measurement errors is subdominant in both regimes; when the weak- and strong-lensing constraints are combined, the amount of degradation due to $\mathcal{C}_{\mathrm{lss}}$ is about $12 \%$ in the total $\mathrm{S} / \mathrm{N}$. Thus, the best-fit parameters are not largely affected by including $\mathcal{C}_{\text {lss }}$, being consistent with each other within statistical uncertainties.

The latter represents projection effects arising from the rich, substructured cluster environment. Recently Meneghetti et al. (2010b) and Rasia et al. (2012) used mock observations of simulated clusters in the $\Lambda \mathrm{CDM}$ cosmology to study the systematic effects in lensing and $\mathrm{X}$-ray-based mass measurements, finding that the standard tangential-shear fitting method, assuming a single spherical NFW profile, can underestimate the true cluster mass $M_{\Delta}$ in the presence of massive substructures, especially for low-mass systems. This is understood by noting that the azimuthally averaged tangential shear probes the differential surface-mass density, $\gamma_{+}(\theta) \propto \bar{\Sigma}(<R)-\Sigma(R)$ (see Equation (5)). Rasia et al. (2012) found from their three most massive systems with $M_{200}>7.5 \times 10^{14} M_{\odot} h^{-1}$ that the level of bias is $\sim-5 \%$ with no noticeable radial dependence at $r=\left(r_{2500}, r_{1000}, r_{500}\right)$. Our cluster mass estimate from the tangential-shear fitting is $M_{\text {vir }}=0.99_{-0.26}^{+0.32} \times 10^{15} M_{\odot} h^{-1}$ (Model 1 of Table 5), which is about 7\% lower than that from our NFW model based on the fulllensing constraints (Model 7 of Table 5) from our comprehensive weak-lensing distortion, magnification, and strong-lensing analysis. This level of underestimation seems to be consistent with the simulation results of Rasia et al. (2012).

\subsubsection{Halo Triaxiality}

A degree of triaxiality is inevitable for collisionless gravitationally collapsed structures (Jing \& Suto 2000; Lemze et al. 2012) and can affect our cluster mass estimation (Oguri et al. 2005; Morandi et al. 2011; Sereno \& Umetsu 2011; Sereno \& Zitrin 2012). In the context of $\Lambda \mathrm{CDM}$, prolate halo shapes are expected to develop by mass accretion along filaments at early stages of halo assembly; hence, dynamically young, clustersized halos tend to have a prolate morphology (Shaw et al. 2006; Lau et al. 2011). Accordingly, a large fraction of clustersized prolate halos, in the absence of selection bias, is expected to be elongated in the plane of the sky (Rasia et al. 2012). On average, this will lead to an underestimation of the cluster mass in a statistical sense when a spherical deprojection (or forward modeling assuming a spherical halo) is applied (Rasia et al. 2012). On the other hand, in the $\Lambda \mathrm{CDM}$ context, those clusters selected by the presence of giant arcs are likely to have their major axes closely aligned with the line of sight (Hennawi et al. 2007; Meneghetti et al. 2010b), because this orientation boosts the projected surface-mass density and hence the lensing signal.

MACS1206 is an X-ray-selected CLASH cluster (Postman et al. 2012), discovered in the MACS survey (Ebeling et al. 2001, 2009). For MACS1206, we find a large projected mass ellipticity of $\left|e_{\Sigma}\right|=1-b / a \gtrsim 0.4$ (or $a / b \gtrsim 1.7$ at $1 \sigma$ ) at large cluster radius $\left(R \gtrsim r_{\text {vir }} \approx 1.6 \mathrm{Mpc} h^{-1}\right)$ based on the Subaru weak-lensing analysis, where its position angle is well aligned with the BCG, optical, X-ray, and LSS shapes in projection space (Section 6.4 and Table 6). The highly elliptical mass distribution in projection would suggest that its major axis is not far from the sky plane and that its true mass and concentration could be even higher than the projected measurements if the cluster size along the sight line is shorter than its effective size scale $(\sqrt{a b})$ in the sky plane.

\subsection{Chandra and XMM-Newton X-Ray Observations}

Complementary multiwavelength observations serve as a useful guide to the likely degree of lensing bias. Here we retrieved and analyzed archival Chandra and XMM-Newton data of MACS1206 to obtain an independent cluster mass estimate, as well as to constrain the physical properties of the X-ray gas. 


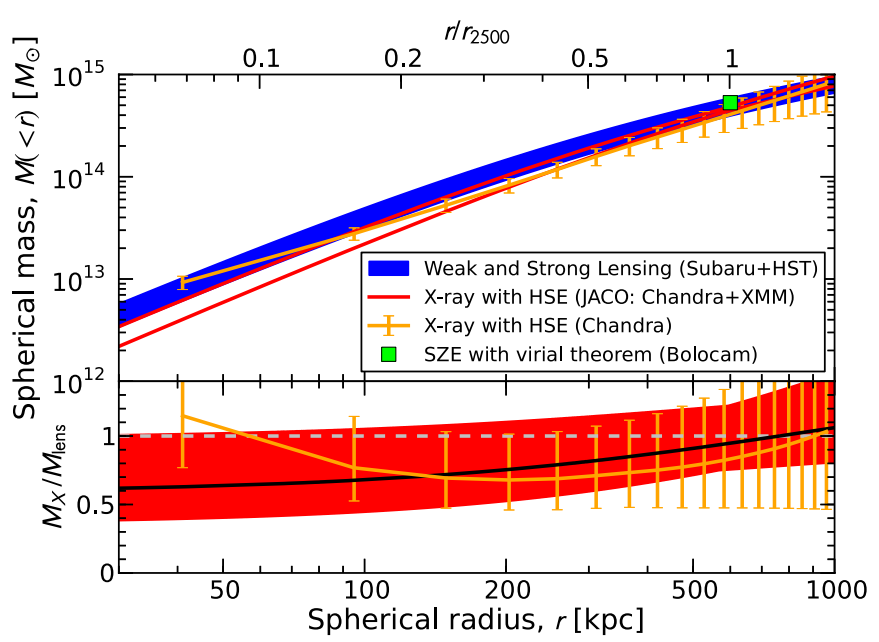

Figure 14. Integrated total mass profiles $M(<r)$ as a function of spherical radius $r$ derived from various observational probes (top). The blue shaded area shows the best-fit NFW model with $1 \sigma$ uncertainty from the combined weak- and strong-lensing measurements (Figures 6 and 7). The red solid lines represent the X-ray-based NFW model ( $1 \sigma$ confidence interval of the fit) derived using the JACO software from a simultaneous fit to Chandra and XMM-Newton observations. The enclosed masses based on Chandra data alone (solid line with error bars, orange) are derived as described in the text, assuming the parameterized pressure profile shape from Arnaud et al. (2010). The green square marks the Bolocam SZE mass estimate at the lensing-derived overdensity radius $r_{2500}$. The bottom panel shows the X-ray-to-lensing mass ratio $M_{X}(<r) / M_{\text {lens }}(<r)$ with $1 \sigma$ uncertainty as a function of radius $r$. The results are shown for both the Chandra-only and joint Chandra+XMM fits.

(A color version of this figure is available in the online journal.)

We perform a simultaneous fit to Chandra and XMM data sets under the assumption that the intracluster gas is in hydrostatic equilibrium (HSE) with the overall cluster potential of the NFW form. The tool used for this analysis is Joint Analysis of Cluster Observations (JACO; Mahdavi et al. 2007b); we refer the reader to this paper for the details of the $\mathrm{X}$-ray analysis procedure, which we briefly summarize below.

We use Chandra ObsID 3277 and XMM-Newton observation 0502430401 . We screen periods of flaring background according to standard procedure, resulting in usable exposure times of $23 \mathrm{ks}$ and $26 \mathrm{ks}$, respectively. Appropriate co-added blanksky fields allow us to subtract particle background spectra for both telescopes, and the residual (positive or negative) astrophysical background is included and marginalized over in the global cluster gas model. Spectra are extracted over seven annular bins for both Chandra and XMM-Newton. The extracted spectra extended out to a distance of 3.7 $(1.26 \mathrm{Mpc})$ and contain an average of 1500 counts each.

The model for the gas density distribution is a single $\beta$-model multiplied by a power law of slope $\gamma$ :

$$
\rho_{g}(r)=\rho_{0}\left(\frac{r_{c}}{r}\right)^{\gamma}\left(1+\frac{r^{2}}{r_{c}^{2}}\right)^{-3 \beta / 2} .
$$

The power-law component is required to capture the steep increase of the density toward the center of the cluster; all parameters of the gas distribution are fit to the data. The metallicity is allowed to vary with radius as well, as are the parameters of the NFW mass profile. Model spectra are generated self-consistently in concentric spherical shells and forward projected onto the annular sky regions matching the extracted annuli. The resulting spectra are mixed using in-orbit energy- and position-dependent PSFs for both Chandra and
Table 10

Comparison with X-Ray Cluster Mass Estimates

\begin{tabular}{lcccc}
\hline \hline Data & $\begin{array}{c}M_{2500} \\
\left(10^{14} M_{\odot}\right)\end{array}$ & $c_{2500}$ & $\begin{array}{c}r_{2500} \\
(\mathrm{Mpc})\end{array}$ & $\begin{array}{c}\theta_{\text {Ein }^{\mathrm{a}}} \\
\left({ }^{\prime \prime}\right)\end{array}$ \\
\hline Chandra & $4 \pm 1$ & $1.8 \pm 1.5$ & $0.6 \pm 0.1$ & 23 \\
Chandra+XMM & $4.5 \pm 0.3$ & $0.9 \pm 0.3$ & $0.58 \pm 0.02$ & 20 \\
WL+SL $^{\mathrm{b}}$ & $4.9 \pm 1.3$ & $1.8 \pm 0.3$ & $0.60 \pm 0.06$ & 28 \\
\hline
\end{tabular}

Notes. See for details Section 7.2. All quantities here are given in physical units assuming the concordance $\Lambda \mathrm{CDM}$ cosmology $\left(h=0.7, \Omega_{m}=0.3, \Omega_{\Lambda}=0.7\right)$.

${ }^{\text {a }}$ Effective Einstein radius for a source at $z_{s}=2.5$ predicted by the model.

b Model 7 of Table 5 based on the full weak- and strong-lensing constraints.

XMM-Newton. Systematic calibration uncertainties between Chandra and XMM-Newton spectra are taken into account by adding a 4\% error (a typical correction used in Mahdavi et al. 2008) in quadrature to each spectral bin used for the joint fits. This brings the joint $\chi^{2}$ into the acceptable range $\left(\chi^{2}=1603\right.$ for 1541 dof). An MCMC procedure is used to estimate errors on the best-fit quantities. After marginalizing over all other parameters, we measure a total mass $M_{2500}=(4.45 \pm 0.28) \times 10^{14} M_{\odot}$, a gas mass $M_{\text {gas }, 2500}=(0.54 \pm 0.02) \times 10^{14} M_{\odot}$, an NFW concentration parameter of $c_{200}=3.5 \pm 0.5$, an inner gas density profile slope of $0.7 \pm 0.03$, and a central cooling time of $2.1 \pm 0.1$ Gyr. In what follows, the examination of the X-ray results is conservatively limited to $r<1 \mathrm{Mpc}$.

In Figure 14 we plot the resulting X-ray-based total mass profile, $M(<r)$, shown along with our NFW model from the full-lensing analysis. The results of the NFW fit are also reported in Table 10. This X-ray model yields a total mass of $M_{X}=(4.6 \pm 0.2) \times 10^{14} M_{\odot}$ at the lensing-derived overdensity radius of $r_{2500} \approx 0.60 \mathrm{Mpc}$. This is in excellent agreement with the lensing mass at the same radius, $M_{\text {lens }}=(4.9 \pm$ $0.9) \times 10^{14} M_{\odot}$, which corresponds to the X-ray-to-lensing mass ratio, $a_{2500}=M_{X}\left(<r_{2500}\right) / M_{\text {lens }}\left(<r_{2500}\right)=0.95_{-0.25}^{+0.23}$. The $a_{2500}$ value obtained here is in good agreement with results from mock observations of $20 \Lambda \mathrm{CDM}$ clusters by Rasia et al. (2012): $a_{2500}=0.94 \pm 0.02$. At this overdensity, no significant bias was observed in detailed observational studies by Zhang et al. (2008) and Mahdavi et al. (2008), who performed a systematic comparison of weak-lensing and X-ray mass measurements for sizable cluster samples. In the bottom panel of Figure 14, we show the X-ray-to-lensing mass ratio $a_{\Delta}$ as a function of cluster radius, in the radial range where $\mathrm{X}$-ray observations are sufficiently sensitive. Overall, the mass ratio is consistent with unity especially at $r \sim r_{2500}$.

Ebeling et al. (2009) obtained a hydrostatic mass estimate of $M_{X}=(1.7 \pm 0.1) \times 10^{15} M_{\odot}$ at $r=2.3 \mathrm{Mpc}$ (their estimate for $r_{200}$ ) assuming an isothermal $\beta$-model with $\beta=0.57 \pm 0.02$ and their estimated temperature $k_{\mathrm{B}} T=11.6 \pm 0.7 \mathrm{keV}$ in the radial range [70, 1000] $\mathrm{kpc}\left(M_{X} \propto \beta^{1 / 2} T\right)$, which is high but consistent within the errors with $M_{\text {lens }}(<2.3 \mathrm{Mpc})=$ $(1.4 \pm 0.3) \times 10^{15} M_{\odot}$ obtained with our best NFW model based on the full-lensing analysis.

Our full-lensing results, when combined with X-ray gas mass measurements $\left(M_{\mathrm{gas}}\right)$, yield a direct estimate for the cumulative gas mass fraction, $f_{\text {gas }}(<r) \equiv M_{\text {gas }}(<r) / M(<r)$, free from the HSE assumption. For this we use reduced Chandra X-ray data presented in the Archive of Chandra Cluster Entropy Profile Tables (ACCEPT; Cavagnolo et al. 2009). In Figure 15, we plot our $f_{\text {gas }}$ measurements as a function of cluster radius. We find a gas mass fraction of $f_{\text {gas }}(<r)=13.7_{-3.0}^{+4.5} \%$ at a radius of $r=1 \mathrm{Mpc} \approx 1.7 r_{2500}\left(\approx 0.8 r_{500}\right)$, a typical value 


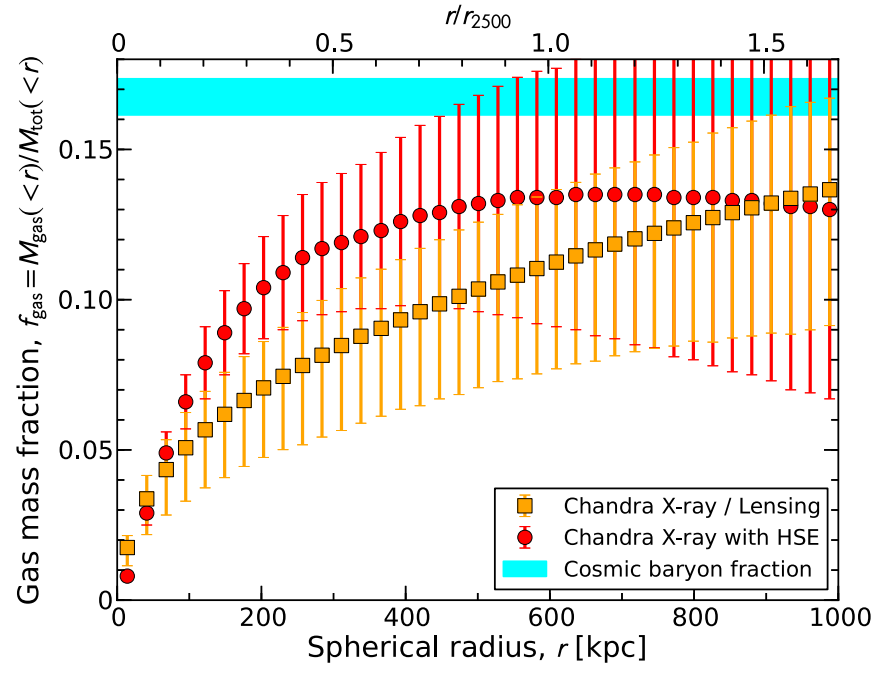

Figure 15. Gas mass fraction profiles $f_{\text {gas }}(<r)=M_{\text {gas }}(<r) / M(<r)$ as a function of spherical radius $r$ derived from joint Subaru weak-lensing, Hubble strong-lensing, and Chandra X-ray observations. In each case the gas mass profile $M_{\text {gas }}(<r)$ is based on the Chandra X-ray data provided in the ACCEPT (Cavagnolo et al. 2009). The squares with error bars represent the results $\left(M_{\text {gas }} / M_{\text {lens }}\right)$ from the combined X-ray and lensing data without employing the hydrostatic equilibrium assumption. The circles with error bars show the Chandra-only results $\left(M_{\mathrm{gas}} / M_{X}\right)$ based on the hydrostatic equilibrium assumption. The horizontal bar shows the constraints $(68 \% \mathrm{CL})$ on the cosmic baryon fraction from the WMAP seven-year data, $f_{b}=\Omega_{b} / \Omega_{m}=$ $0.1675 \pm 0.006$.

(A color version of this figure is available in the online journal.)

observed for high-mass clusters (Umetsu et al. 2009; Zhang et al. 2009). When compared to the cosmic baryon fraction $f_{b}=\Omega_{b} / \Omega_{m}=0.1675 \pm 0.006$ constrained from the Wilkinson Microwave Anisotropy Probe (WMAP) seven-year data (Jarosik et al. 2011), this indicates $f_{\text {gas }} / f_{b}=0.82_{-0.18}^{+0.27}$ at this radius. At the innermost measurement radius $r \approx 40 \mathrm{kpc}$ where the lensing and X-ray data overlap, we have $f_{\text {gas }}(<r)=3.4_{-0.8}^{+1.2} \%$. Thus, the hot gas represents only a minor fraction of the total lensing mass near the cluster center, as found for other highmass clusters (Lemze et al. 2008; Umetsu et al. 2009).

Additionally, we derive a mass profile from simulated annealing fits of the ACCEPT pressure profile (Cavagnolo et al. 2009), adopting the Arnaud et al. (2010, A10) "universal profile" (M. Donahue et al. 2012, in preparation). This Chandra-only mass profile is shown to be in good agreement with the lensing as well as joint Chandra+XMM results (Figure 14). The Chandra-only gas mass fraction profile is also shown in Figure 15.

We conclude, on the basis of these results and comparison with detailed statistical studies, that the level of orientation bias in this cluster is not significant given the large uncertainties in our lensing/X-ray observations, as well as the possible contribution from non-thermal pressure in the cluster core (e.g., Kawaharada et al. 2010).

\subsection{Bolocam SZE Observations}

We have also compared our lensing-derived results to mass estimates determined from the SZE data. Using Bolocam at the Caltech Submillimeter Observatory, we observed MACS1206 for approximately $11 \mathrm{hr}$ in 2011 April. These data were collected with Bolocam configured at an SZE-emission-weighted band center of $140 \mathrm{GHz}$. Further details of the Bolocam instrument are given in Haig et al. (2004). We detect the cluster with an $\mathrm{S} / \mathrm{N}$ value of 21.1 and a white noise rms of $24.9 \mu \mathrm{K}_{\mathrm{CMB}}$ arcmin.
Table 11

Bolocam SZE-derived Cluster Mass Estimates

\begin{tabular}{lccccc}
\hline \hline Overdensity $^{\mathrm{a}}$ & \multicolumn{2}{c}{ Bolocam-derived $r_{\Delta}{ }^{\mathrm{b}}$} & & \multicolumn{2}{c}{ Lensing-derived $r_{\Delta}{ }^{\mathrm{c}}$} \\
\cline { 2 - 3 } & $\begin{array}{c}r_{\Delta} \\
(\mathrm{Mpc})\end{array}$ & $\begin{array}{c}M\left(<r_{\Delta}\right) \\
\left(10^{14} M_{\odot}\right)\end{array}$ & & $\begin{array}{c}r_{\Delta} \\
(\mathrm{Mpc})\end{array}$ & $\begin{array}{c}M\left(<r_{\Delta}\right) \\
\left(10^{14} M_{\odot}\right)\end{array}$ \\
\hline 2500 & $0.63_{-0.02-0.05}^{+0.01+0.06}$ & $5.8_{-0.4-1.4}^{+0.4+1.7}$ & & 0.60 & $5.3_{-0.2-0.7}^{+0.2+0.8}$ \\
500 & $1.67_{-0.08-0.12}^{+0.09+0.12}$ & $21.2_{-3.0-4.3}^{+3.7+5.1}$ & & 1.31 & $15.7_{-1.1-2.1}^{+1.2+2.3}$ \\
\hline
\end{tabular}

Notes. For each value the first error estimate represents our measurement uncertainty and the second error estimate represents our uncertainty due to systematics in our fitting method, flux calibration, and choice of parameterization. See for details Section 7.3. All quantities here are given in physical units assuming the concordance $\Lambda$ CDM cosmology $\left(h=0.7, \Omega_{m}=0.3, \Omega_{\Lambda}=0.7\right)$.

a Mean interior overdensity with respect to the critical density of the universe at the cluster redshift $z=0.439$.

b Bolocam cluster mass estimates at the Bolocam-SZE-derived values of overdensity radius $r_{\Delta}$.

c Bolocam cluster mass estimates at the lensing-derived values of overdensity radius $r_{\Delta}$.

We reduced these data according to the procedure described in detail in Sayers et al. (2011), but with the updated calibration model reported in Sayers et al. (2012) and some other minor modifications.

The key steps involved in our Bolocam data reduction and cluster modeling are summarized as follows. We first remove sky noise from the time streams by subtracting a template of the correlated signal over the field of view followed by a high-pass filter (Sayers et al. 2011). This process results in a filtered image of the true SZE signal (see the left panel in Figure 16), where the filtering is weakly dependent on the cluster shape due to the correlated template removal. To characterize this filtering, we process a beam-smoothed, initial best-fit cluster profile by reverse mapping it using our pointing information. These data are then processed iteratively with a new best-fit profile using our full reduction pipeline, until the procedure converges. For this analysis we use the A10 "universal pressure profile," which adopts a form of the Nagai et al. (2007) pressure profile with its slopes fixed to the values given in A10, allowing the overall normalization and scale radius to vary.

We have derived cluster mass estimates from our SZE data alone using the method outlined in Mroczkowski (2011). The key innovation of this method is that, in addition to assuming HSE, the virial theorem is used, which is no stronger an assumption than HSE and can be derived from HSE and thermodynamics. This method determines the underlying total mass profile from an SZE-determined pressure profile, with the added assumption of a constant gas mass fraction $f_{\text {gas }}$. Cluster mass estimates derived with this method have been shown to be consistent with X-ray-derived results using data from the SZA (Mroczkowski 2011) and SZA follow-up of blind SZE detections using the Atacama Cosmology Telescope (Reese et al. 2012). The SZE-only mass estimates for MACS1206 are given in Table 11, which presents $M_{\Delta}$ and $r_{\Delta}$ values at overdensities $\Delta=2500$ and 500 derived from our Bolocam data alone, under the assumptions made. This table also contains Bolocam-derived mass estimates at the lensing-derived values of $r_{2500}$ and $r_{500}$. We note that the values in Table 11 include an estimate of our systematic errors on the SZE-derived masses, which we describe in detail below.

The dominant source of uncertainty in our mass estimates, as discussed in Mroczkowski (2011), stems from the uncertainty in the assumed value of a radially constant $f_{\text {gas }}(r)$. Masses 


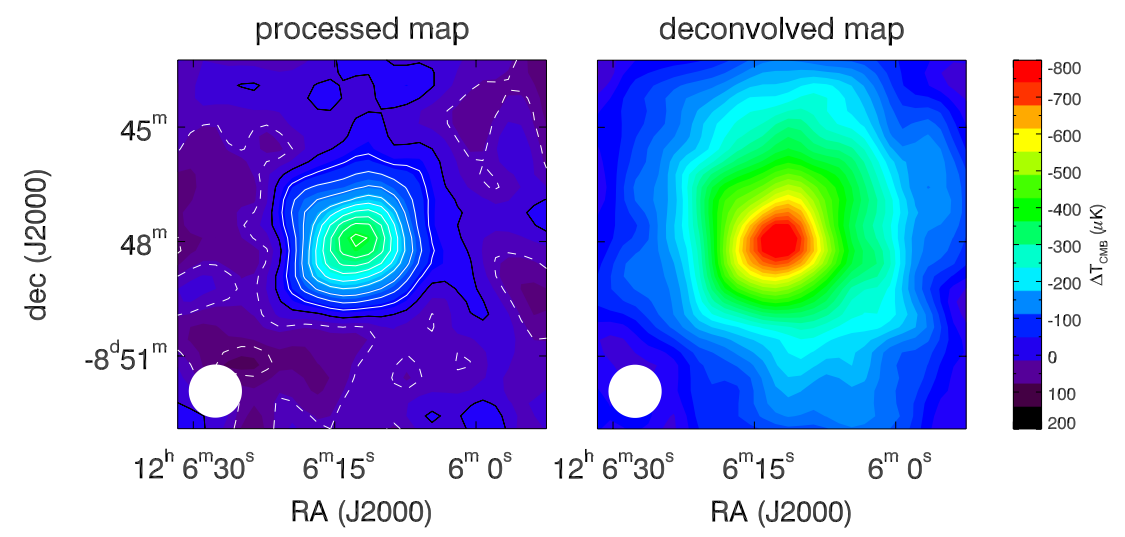

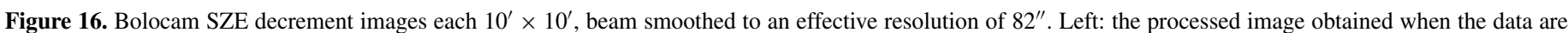

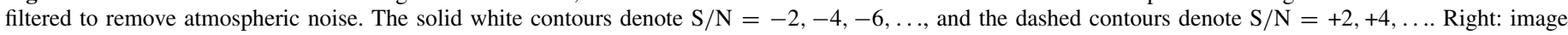
obtained when the effects of the atmospheric filtering have been deconvolved to obtain an unbiased image of the cluster.

(A color version of this figure is available in the online journal.)

derived under this assumption scale as $\propto f_{\mathrm{gas}}^{-1 / 2}$. We adopt the value $f_{\text {gas }}=0.13$ and marginalize over uncertainties for a range $f_{\text {gas }}=[0.1,0.17]$, consistent with our X-ray-determined gas fraction measurements at radii near $r_{2500}$ (see Figure 15). An additional source of systematic uncertainty is the absolute calibration of the Bolocam maps, which is about $5 \%$ and results in a $\lesssim 5 \%$ uncertainty in our derived masses. Finally, we include a $\pm 1.5 \%$ systematic at $r_{2500}$ and $\pm 5 \%$ systematic at $r_{500}$, due to our particular choice of parameterization for the pressure profile. These values are roughly consistent with those shown in Mroczkowski (2011) for different parameterization of the exponents in the pressure profiles.

By comparison to the lensing mass estimates, we find an SZE-to-lensing mass ratio of $a_{2500}=1.08 \pm 0.29 \pm 0.22$ (statistical followed by systematic at $68 \%$ confidence) at the lensing-derived overdensity radius $r_{2500}$ of $0.60 \mathrm{Mpc}$ (Table 11). Hence, our lensing mass estimate is in agreement with both the X-ray and SZE mass estimates at $r_{2500}$. At a lower overdensity of $\Delta=500$, we find an SZE-to-lensing mass ratio of $a_{500}=1.55 \pm 0.30 \pm 0.26$ at the lensing-derived radius $r_{500}$ of $1.3 \mathrm{Mpc}$, roughly consistent with unity within large errors.

\subsection{Dynamical and Physical Conditions of the Cluster}

MACS1206 is an X-ray-luminous cluster at a redshift of $z=0.439$, or a cosmic time of $t \sim 9$ Gyr. The cluster appears relatively relaxed in projection in both optical and X-ray images, with a pronounced X-ray peak at the BCG position (Ebeling et al. 2009; Postman et al. 2012). This cluster was classified to be relaxed by Gilmour et al. (2009) on the basis of a visual examination of its X-ray morphology. Our detailed morphology analysis shows no sign of significant recent merging activity around the BCG, which is also supported by our strong-lensing analysis, finding no significant offset between the DM center of mass, BCG, and X-ray peak (Section 4.2). A good agreement between the lensing and X-ray mass estimates (Section 7.2) indicates that the hot gas is not far from a state of HSE in the cluster potential well.

However, some evidence of merger activity along the line of sight was suggested by the high velocity dispersion of $\sigma_{v} \approx 1580 \mathrm{~km} \mathrm{~s}^{-1}$ based on 38 redshift measurements (Ebeling et al. 2009). Recently, a much larger spectroscopic sample of cluster members has been obtained for this cluster using VLT/VIMOS (P. Rosati et al. 2012, in preparation). Defining membership is crucial for a dynamical analysis since interlopers by projection effects can largely bias the derived projected velocity dispersion, especially at large radii where the number density of cluster members is low (Wojtak et al. 2007). Using a secure sample of $>400$ cluster members identified in the projected phase space (e.g., Biviano \& Salucci 2006; Lemze et al. 2009), we find that the velocity dispersion profile decreases outward fairly rapidly from $\sim 1500 \mathrm{~km} \mathrm{~s}^{-1}$ in the central region to $\sim 800 \mathrm{~km} \mathrm{~s}^{-1}$ at a projected distance of $R \sim 2 \mathrm{Mpc}$. Accordingly, the dynamical mass estimate is in agreement with the lensing estimate (A. Biviano et al. 2012, in preparation). This may argue against a strong deviation from dynamical relaxation.

The present Chandra analysis yields a gas temperature of $10.8 \pm 0.7 \mathrm{keV}$ averaged in the radial range [70,700] $\mathrm{kpc}$. Assuming that the galaxies and the gas are confined in the same gravitational potential well, this is consistent with a lineof-sight velocity dispersion of $\sim 1300 \mathrm{~km} \mathrm{~s}^{-1}$, which is again in agreement with the observed value. This may also suggest that the cluster is not far from equilibrium. The cluster appears fairly round in both Chandra and XMM images at large distances from the cluster center, as demonstrated in Figure 10. X-ray emission is concentrated around and peaked on the BCG but shows some elongation within $\theta \lesssim 1^{\prime}$ at a position angle around $120^{\circ}$ east of north, aligned with the orientation of the projected mass distribution. The surface brightness profile is fairly smooth, but there might be some tiny hints of discontinuities (see the ACCEPT catalog). ${ }^{48}$ However, a much deeper observation is required to confirm them.

Finally, morphological analysis of Bolocam data has been performed in an identical way to the procedure used in Sayers et al. (2011). We find an ellipticity of $(10 \pm 7) \%$ with a position angle of $55^{\circ} \pm 27^{\circ}$ north of west from elliptical A10 model fits to our Bolocam SZE data. The fits include all data within a $14 \times 14$ arcmin square, corresponding to a fairly large aperture of $\theta_{\max } \approx 9^{\prime}>\theta_{\mathrm{vir}} \sim 7^{\prime}$. Of the approximately 50 clusters observed with Bolocam and fit with an elliptical A10 model, MACS1206 is one of the more circularly symmetric model fits.

\subsection{Comparison with $\Lambda C D M$ Predictions}

In Figure 17, we summarize our full-lensing constraints on the mass and concentration parameters of MACS1206, along with recent $\Lambda \mathrm{CDM}$ predictions for relaxed cluster-sized halos

\footnotetext{
48 http://www.pa.msu.edu/astro/MC2/accept/clusters/3277.html
} 


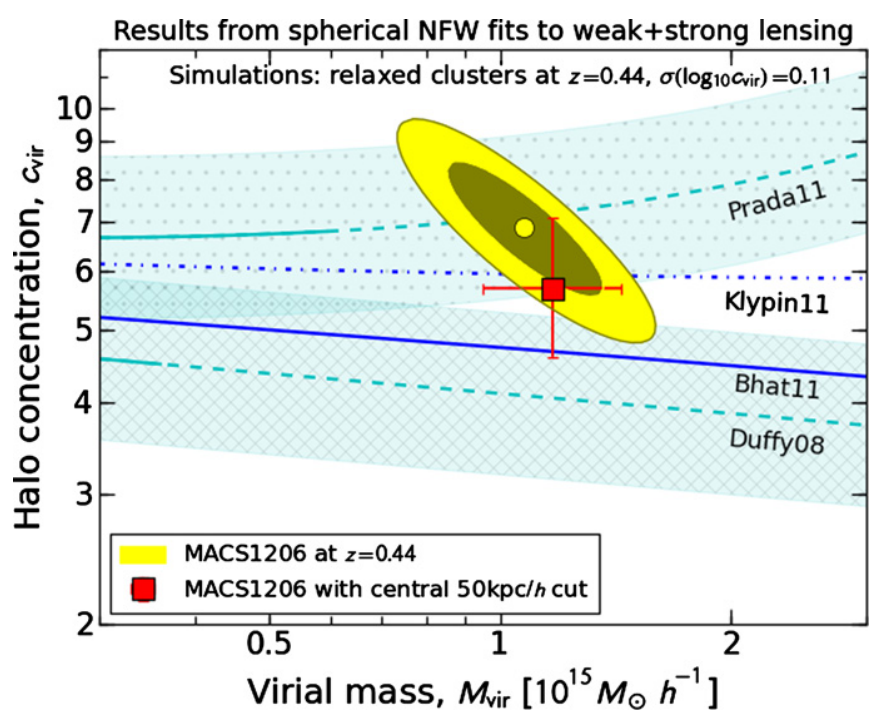

Figure 17. Constraints on the halo mass and concentration parameters $\left(M_{\mathrm{vir}}, c_{\mathrm{vir}}\right)$ for the X-ray-selected CLASH cluster MACS1206 $(z=0.439)$ derived from spherical NFW fits to combined weak- and strong-lensing observations, compared to $\Lambda \mathrm{CDM}$ predictions for relaxed populations of simulated cluster-sized halos at $z=0.44$ (except $z=0.5$ for Klypin et al. 2011). Our results are shown with and without the central $50 \mathrm{kpc} h^{-1}$ cut (Section 6.6) applied to the Zitrin et al. (2012) based strong-lensing model. The $N$-body predictions by Duffy et al. (2008) and Prada et al. (2011) are shown in light blue, including $1 \sigma$ lognormal scatter $\left(0.11\right.$ in $\left.\log _{10} c_{\mathrm{vir}} ; \sim 29 \%\right)$ indicated by the respective hatched areas. Portions of these lines are dashed to indicate extrapolations to higher masses. Average results from two additional simulations (Klypin et al. 2011; Bhattacharya et al. 2011) are shown in blue for clarity. Duffy et al. (2008) and Bhattacharya et al. (2011) derived results for dynamically relaxed cluster subsamples, yielding concentrations $\sim 10 \%$ higher than for the full samples. This $10 \%$ factor has been applied to the results from the other simulations.

(A color version of this figure is available in the online journal.)

based on $N$-body simulations (Duffy et al. 2008; Klypin et al. 2011; Bhattacharya et al. 2011; Prada et al. 2011). Our range of allowed concentration values $\left(4.6 \leqslant c_{\text {vir }} \leqslant 7.9\right.$ at $1 \sigma$; see Section 6.6) span the high end and average expectations $\left(4 \lesssim\left\langle c_{\text {vir }}\right\rangle \lesssim 7\right)$ from $\Lambda$ CDM simulations (Duffy et al. 2008; Zhao et al. 2009; Klypin et al. 2011; Bhattacharya et al. 2011; Prada et al. 2011). Average concentrations for relaxed clusters are found to be $\sim 10 \%$ higher and have lower scatter than those for the full population of halos (Duffy et al. 2008; Bhattacharya et al. 2011). A relatively high concentration of MACS1206 may also be indicated by the large Einstein radius $\theta_{\text {Ein }} \approx 28^{\prime \prime}\left(17^{\prime \prime}\right)$ at $z_{s}=2.5$ (1.0) (Ebeling et al. 2009; Zitrin et al. 2012).

Care must be taken when comparing these predictions for spherically averaged halo structure with our lensing results, which are obtained from an NFW fit to the projected lensing measurements assuming a spherical halo. In the previous subsection (Section 7.1), we have shown that our lensing results are in good agreement with the X-ray-derived mass profiles (see Figures 14 and 15) in the region of overlap ( $\lesssim 1 \mathrm{Mpc})$, as well as with the Bolocam SZE mass estimates (Section 7.3), suggesting that the level of orientation bias (see Section 7.1.2) is not significant in this cluster.

Additionally, the effects of baryonic physics can impact the inner halo profile (at $r \lesssim 0.05 r_{\text {vir }}$; Duffy et al. 2010) and thus modify the gravity-only $c-M$ relation, especially for less massive halos $\left(M_{\text {vir }} \lesssim 4 \times 10^{14} M_{\odot} h^{-1}\right.$; see Bhattacharya et al. 2011). Using cosmological hydrodynamical simulations including the back-reaction of baryons on DM, Duffy et al. (2010) found a $<20 \%$ increase in the halo concentration for cluster-sized halos $\left(M_{\mathrm{vir}}<6 \times 10^{14} M_{\odot} h^{-1}\right.$ at $\left.z=0\right)$. When excluding the central $50 \mathrm{kpc} h^{-1}\left(\approx 0.03 r_{\text {vir }}\right)$ region from our primary strong-lensing mass model (Zitrin et al. 2012), we find a $\approx 17 \%$ decrease in the best-fit concentration parameter derived from our full-lensing analysis (Section 6.6), as demonstrated in Figure 17. We note that the CLASH clusters are massive $\left(5 \times 10^{14}<M_{\text {vir }} / M_{\odot}<3 \times 10^{15}\right.$; see Postman et al. 2012) and hence expected to be less affected by baryonic effects.

For this cluster, the lensing-derived total mass distribution is consistent with the NFW form $\left(\alpha=\gamma_{3 \mathrm{D}}(r \rightarrow 0)=0.96_{-0.49}^{+0.31}\right)$, as found for several relaxed clusters: A611 (Newman et al. 2009); A383 (Zitrin et al. 2011c); A1703 ( $\alpha \approx 0.9$ Richard et al. 2009; Oguri et al. 2009); a stacked full-lensing analysis of A1689, A1703, A370, and $\mathrm{Cl} 0024+17\left(\alpha=0.89_{-0.39}^{+0.27}\right.$; Umetsu et al. 2011a). Multiwavelength observations can be used to measure gas and stellar density profiles for subtraction from lensing-derived total mass profiles to yield DM-only mass profiles (Lemze et al. 2008; Newman et al. 2009), allowing for a more direct comparison with CDM predictions from gravityonly simulations. We defer this analysis to a forthcoming paper.

\section{SUMMARY AND CONCLUSIONS}

In this paper, we have presented a comprehensive lensing analysis, combining independent measurements of weaklensing distortion, magnification, and strong lensing of the massive X-ray-selected cluster MACS1206 at $z=0.439$. This is based on wide-field Subaru $B V R_{\mathrm{c}} I_{\mathrm{c}} z^{\prime}$ imaging, combined with detailed strong-lensing information obtained from deep CLASH HST 16-band imaging and VLT/VIMOS spectroscopy (Zitrin et al. 2012).

The deep Subaru multi-band photometry is used to separate background, foreground, and cluster galaxy populations using the selection techniques established in our earlier work (Medezinski et al. 2010; Umetsu et al. 2010), allowing us to obtain a reliable weak-lensing signal free from significant contamination of unlensed cluster and foreground galaxies. By combining complementary distortion and magnification measurements, we constructed a model-free mass distribution out to well beyond the virial radius $\left(r_{\text {vir }} \approx 1.6 \mathrm{Mpc} h^{-1}\right)$. In addition to breaking the mass-sheet degeneracy inherent in shape distortion measurements, the magnification measurements also increase the overall significance by $\sim 23 \%$ (Section 5.3).

We have also obtained an improved inner mass distribution from a reanalysis of the Zitrin et al. (2012) data using our new MCMC implementation of the Zitrin et al. (2009) method. We introduced a technique to self-calibrate the bin-to-bin covariance matrix of the inner mass profile (Section 4.3), accounting for possible systematic errors inherent in the analysis. This is a crucial step for a joint analysis to combine constraints in different regimes of signal strength. The inner radial boundary for the mass profile is chosen to be sufficiently large to avoid smoothing from cluster miscentering effects (Johnston et al. 2007). The derived inner mass profile is shown to be consistent with our semi-independent results from a wide variety of four strong-lensing analyses (Lenstool, Pixelens, LensPerfect, and SAWLENS; see Section 4.4) and to overlap well with the Subaru-based outer mass profile, ensuring consistency in both the weak and strong regime.

The Subaru data reveal the presence of an elongated LSS around the cluster, both in the distribution of galaxies and from the mass distribution, with the major axis running NW-SE, aligned well with the cluster and BCG shapes, showing 
elongation with a $\sim 2: 1$ axis ratio in the plane of the sky (Section 6.2). The azimuthally averaged projected mass profile from our full-lensing analysis exhibits a shallow profile slope $d \ln \Sigma / d \ln R \sim-1$ at cluster outskirts $\left(R \gtrsim 1 \mathrm{Mpc} h^{-1}\right)$, whereas the mass distribution excluding the NW-SE excess regions steepens further out, well described by the standard NFW form (Section 6.4). Assuming a spherical halo, we have obtained a virial mass $M_{\text {vir }} / 10^{15} M_{\odot} h^{-1}=1.07_{-0.16}^{+0.20}$ (stat.) \pm 0.10 (syst.) and a halo concentration $c_{\text {vir }}=6.9_{-0.9}^{+1.0}$ (stat.) \pm 1.2 (syst.), which is somewhat high but falls in the range $4 \lesssim\langle c\rangle \lesssim 7$ of average $c(M, z)$ predictions for relaxed clusters from recent $\Lambda \mathrm{CDM}$ simulations. When the innermost $50 \mathrm{kpc} h^{-1}$ is excluded from the fit, we find a slightly lower concentration $c_{\text {vir }}=5.7_{-1.1}^{+1.4}$ (stat.) \pm 1.2 (syst.), a decrease of approximately $17 \%$ (Section 6.6).

We have shown that our full-lensing mass profile is in agreement with Chandra+XMM X-ray data in the region of overlap (Figure 14). The hydrostatic X-ray-to-lensing mass ratio, $a_{\Delta}=M_{X}\left(<r_{\Delta}\right) / M_{\text {lens }}\left(<r_{\Delta}\right)$, is consistent with unity especially at $r \sim r_{2500}$ with $a_{2500}=0.95_{-0.25}^{+0.23}$. Our full-lensing results, when combined with Chandra gas mass measurements, yield a gas mass fraction estimate free from the HSE assumption. We find a cumulative gas mass fraction of $f_{\text {gas }}(<r)=13.7_{-3.0}^{+4.5} \%$ at $r \approx 1.7 r_{2500}$, a typical value observed for high-mass clusters. Overall good agreement is also obtained with SZE-only cluster mass estimates based on Bolocam observations (Section 7.3).

The CLASH survey is producing substantial improvements in both the quality and quantity of direct empirical constraints on cluster-sized DM halos (Postman et al. 2012; Zitrin et al. 2011c; Coe et al. 2012; Zheng et al. 2012), for an X-ray-selected sample of relaxed clusters, selected free of lensing selection bias, as well as for a lensing-selected sample of high-magnification clusters. The CLASH imaging, in combination with Subaru weak-lensing data, allows us to make precise measurements of the mass distributions of individual clusters over the full range of cluster radius and to help understand the possible evolutionary and tidal effects of connecting filaments and local clusters on the mass distribution of the central cluster, for a detailed comparison with the standard $\Lambda \mathrm{CDM}$ cosmology and a wider examination of alternative scenarios. With the full sample of CLASH clusters, we will be able to establish the representative mass profile of massive clusters in gravitational equilibrium and robustly test models of structure formation.

We thank our referee for a careful reading of the manuscript and for providing useful comments. We acknowledge useful discussions with Nobuhiro Okabe, Masamune Oguri, and Mauro Sereno. We are grateful for comments by Cheng-Jiun Ma. We thank Nick Kaiser for making the IMCAT package publicly available. We thank G. Mark Voit for having contributed to the ACCEPT-based X-ray mass measurements in advance of publication. We are grateful for the hospitality of the Spitzer Science Center at Caltech, where part of this work was done.

The CLASH Multi-Cycle Treasury Program is based on observations made with the NASA/ESA Hubble Space Telescope. The Space Telescope Science Institute is operated by the Association of Universities for Research in Astronomy, Inc., under NASA contract NAS 5-26555. ACS was developed under NASA contract NAS 5-32864. This research is supported in part by NASA grant HST-GO-12065.01-A, National Science Council of Taiwan grant NSC100-2112-M-001-008-MY3, and PRIN INAF 2010. K.U. acknowledges support from the Academia Sinica Career Development Award. Part of this work is based on data collected at the Very Large Telescope at the ESO Paranal Observatory, under Programme ID 186.A-0798. P.R., C.G., I.B., and S.S. acknowledge partial support by the DFG cluster of excellence Origin and Structure of the Universe. The Bolocam observations were partially supported by the Gordon and Betty Moore Foundation. J.S. was supported by NSF/AST0838261 and NASA/NNX11AB07G; N.C. was partially supported by a NASA Graduate Student Research Fellowship. A.Z. is supported by the "Internationale Spitzenforschung II/2" of the Baden-Württemberg Stiftung. C.G. acknowledges support from the Dark Cosmology Centre, which is funded by the Danish National Research Foundation. I.S. holds a PhD FPI Fellowship contract from the Spanish Ministry of Economy and Competitiveness and is also supported by the mentioned ministry through research project FIS2010-15492. Support for T.M. was provided by NASA through the Einstein Fellowship Program, grant PF0-110077.

\section{APPENDIX}

\section{COMBINING LENS DISTORTION AND MAGNIFICATION}

\section{A.1. One-dimensional Method}

We first summarize the Bayesian method of Umetsu et al. (2011b) for a direct reconstruction of the cluster mass profile from combined radial distortion and magnification profiles.

In the Bayesian framework, we sample from the posterior pdf of the underlying signal $s$ given the data $\boldsymbol{d}, P(\boldsymbol{s} \mid \boldsymbol{d})$. Expectation values of any statistic of the signal $s$ shall converge to the expectation values of the a posteriori marginalized pdf, $P(\boldsymbol{s} \mid \boldsymbol{d})$. For a mass profile analysis, $\boldsymbol{s}$ is a vector containing the discrete convergence profile, $\kappa_{i} \equiv \kappa\left(\theta_{i}\right)$ with $i=1,2, \ldots, N$ in the subcritical regime $\left(\theta_{i}>\theta_{\text {Ein }}\right)$, and the average convergence within the inner radial boundary $\theta_{\min }$ of the weak-lensing data, $\bar{\kappa}_{\text {min }} \equiv \bar{\kappa}\left(<\theta_{\text {min }}\right)$, so that $s=\left\{\bar{\kappa}_{\min }, \kappa_{i}\right\}_{i=1}^{N}$, being specified by $(N+1)$ parameters.

Bayes' theorem states that

$$
P(\boldsymbol{s} \mid \boldsymbol{d}) \propto P(\boldsymbol{s}) P(\boldsymbol{d} \mid \boldsymbol{s}),
$$

where $\mathcal{L}(\boldsymbol{s}) \equiv P(\boldsymbol{d} \mid \boldsymbol{s})$ is the likelihood of the data given the model $(s)$ and $P(s)$ is the prior probability distribution for the model parameters. The $\mathcal{L}(s)$ function for combined weaklensing observations is given as a product of the two separate likelihoods, $\mathcal{L}=\mathcal{L}_{g_{+}} \mathcal{L}_{\mu}$, where $\mathcal{L}_{g_{+}}$and $\mathcal{L}_{\mu}$ are the likelihood functions for tangential distortion and magnification bias, respectively. The log-likelihood functions for the weak-lensing observations $\left\{g_{+, i}\right\}_{i=1}^{N}$ and $\left\{n_{\mu, i}\right\}_{i=1}^{N}$ are given, respectively (ignoring constant terms), as

$$
\begin{aligned}
& l_{g_{+}}(s) \equiv-\ln \mathcal{L}_{g}=\frac{1}{2} \sum_{i=1}^{N} \frac{\left[g_{+, i}-\hat{g}_{+, i}(s)\right]^{2}}{\sigma_{+, i}^{2}}, \\
& l_{\mu}(s) \equiv-\ln \mathcal{L}_{\mu}=\frac{1}{2} \sum_{i=1}^{N} \frac{\left[n_{\mu, i}-\hat{n}_{\mu, i}(s)\right]^{2}}{\sigma_{\mu, i}^{2}},
\end{aligned}
$$

where $\left\{\hat{g}_{+, i}\right\}_{i=1}^{N}$ and $\left\{\hat{n}_{\mu, i}\right\}_{i=1}^{N}$ are the theoretical predictions for the corresponding observations. The total likelihood $l_{1 \mathrm{D}}(s) \equiv$ $-\ln \mathcal{L}$ of the combined observations is obtained as

$$
l_{1 \mathrm{D}}=l_{g_{+}}+l_{\mu}
$$

Here we consider a simple flat prior with a lower bound of $s=0$. Additionally, we account for the uncertainty in the calibration 
parameters, $c=\left(n_{0}, s, \omega\right)$, namely, the normalization and slope parameters $\left(n_{0}, s\right)$ of the background counts and the relative lensing depth $\omega \equiv\langle\beta$ (red) $\rangle /\langle\beta$ (back) $\rangle$ between the background samples used for the magnification and distortion measurements.

We use the MCMC technique with Metropolis-Hastings sampling to constrain our mass model $s$. The covariance matrix $\mathcal{C}$ of $s$ is obtained from MCMC samples.

\section{A.2. Two-dimensional Method}

Here we extend the 1D method of Umetsu et al. (2011b) to a 2D mass distribution $\kappa(\boldsymbol{\theta})$, by combining 2D distortion data with the azimuthally averaged magnification information. For this analysis, the signal $s$ is a vector of parameters containing discrete mass elements on a 2D Cartesian grid of independent cells: $\boldsymbol{s}=\left\{\kappa_{m}\right\}_{m=1}^{N_{\text {cell }}}$. The $\gamma(\boldsymbol{\theta})$ field can be written as a linear combination of the parameters $s$ (Equation (3)). Then, the distortion $g(\boldsymbol{\theta})$ and magnification $\mu(\boldsymbol{\theta})$ fields can be uniquely specified in the subcritical regime (Section 2$)$.

In analogy to Equation (13), we calculate the weighted average $g_{\alpha, m} \equiv g_{\alpha}\left(\boldsymbol{\theta}_{m}\right)(\alpha=1,2)$ of individual distortion estimates and its covariance matrix,

$$
\operatorname{Cov}\left[g_{\alpha, m}, g_{\beta, n}\right] \equiv\left(C_{g}\right)_{\alpha \beta, m n}=\frac{1}{2} \sigma_{g}^{2}\left(\boldsymbol{\theta}_{m}\right) \delta_{m n} \delta_{\alpha \beta},
$$

where $\sigma_{g}^{2}\left(\boldsymbol{\theta}_{m}\right)$ is the standard error of the weighted mean distortion, $g\left(\boldsymbol{\theta}_{m}\right)$. Accordingly, the 2D shear log-likelihood function $l_{g}(s) \equiv-\ln \mathcal{L}_{g}$ is written as

$l_{g}(\boldsymbol{s})=\frac{1}{2} \sum_{m, n=1}^{N_{\text {cell }}} \sum_{\alpha, \beta=1}^{2}\left[g_{\alpha, m}-\hat{g}_{\alpha, m}(\boldsymbol{s})\right]\left(\mathcal{W}_{g}\right)_{\alpha \beta, m n}\left[g_{\beta, n}-\hat{g}_{\beta, n}(\boldsymbol{s})\right]$,

where $\hat{g}_{\alpha, m}(s)$ is the theoretical prediction for $g_{\alpha, m}$ and $\left(\mathcal{W}_{g}\right)_{\alpha \beta, m n}$ is the shear weight matrix,

$$
\left(\mathcal{W}_{g}\right)_{\alpha \beta, m n}=M_{m} M_{n}\left(\mathcal{C}_{g}^{-1}\right)_{\alpha \beta, m n}
$$

with $M_{m}$ being a mask weight, defined such that $M_{m}=0$ if the $m$ th cell is masked out and $M_{m}=1$ otherwise. In practice, we exclude from our analysis innermost cells that lie in the cluster central region, where the surface-mass density can be close to or greater than the critical value (i.e., $\kappa \gtrsim 1$ ). Furthermore, this is crucial to minimize contamination by unlensed cluster member galaxies (see Section 3.4).

Now we combine 2D distortion data with magnification information to obtain the total $\log$-likelihood $l_{2 \mathrm{D}}(s)$ as

$$
l_{2 \mathrm{D}}=l_{g}+l_{\mu},
$$

where $l_{\mu}$, given by Equation (A3), imposes a set of azimuthally integrated constraints on the underlying $\kappa$ field. Since the degree of magnification is locally related to $\kappa$, this will essentially provide the (otherwise unconstrained) normalization of $\kappa(\boldsymbol{\theta})$ over a set of concentric rings where count measurements $n_{\mu, i}$ are available. Note that no assumption is made of azimuthal symmetry or isotropy of the cluster mass distribution.

This 2D inversion problem involves estimation of a large number of parameters $s$; typically, $N_{\text {cell }} \gtrsim 1000$ when distortion data are binned into subarcminute pixels. We use in our implementation the conjugate-gradient method (Press et al. 1992) to find the best solution. We include Gaussian priors on the calibration nuisance parameters $c=\left(s, n_{0}, \omega\right)$, given by means of quadratic penalty terms with mean values and variances directly estimated from data. The log posterior pdf, $F=-\ln P(\boldsymbol{s} \mid \boldsymbol{d})$, is expressed as a linear sum of $l_{2 \mathrm{D}}(\boldsymbol{s})$ and the prior terms on $\boldsymbol{c}$. The best-fit parameters are determined with a maximum-likelihood estimation, by minimizing the function $F$ with respect to $p \equiv(s, c)$, a vector containing the mass and calibration parameters. Here we employ an analytic expression for the gradient function $\nabla F(\boldsymbol{p})$ obtained in the nonlinear subcritical regime. To quantify the errors on the mass reconstruction, we evaluate the Fisher matrix at the maximumlikelihood estimate $\boldsymbol{p}=\hat{\boldsymbol{p}}$ as

$$
\mathcal{F}_{m n}=\left.\left\langle\frac{\partial^{2} F(\boldsymbol{p})}{\partial p_{m} \partial p_{n}}\right\rangle\right|_{\boldsymbol{p}=\hat{\boldsymbol{p}}},
$$

where the angular brackets represent an ensemble average and the indices $(m, n)$ run over all model parameters. We estimate the covariance matrix $\mathcal{C}$ of $s$ as

$$
\mathcal{C}_{m n}=\left(\mathcal{F}^{-1}\right)_{m n} .
$$

\section{REFERENCES}

Allen, S. W., Schmidt, R. W., Ebeling, H., Fabian, A. C., \& van Speybroeck, L. 2004, MNRAS, 353, 457

Arnaud, M., Pratt, G. W., Piffaretti, R., et al. 2010, A\&A, 517, A92

Bartelmann, M., \& Schneider, P. 2001, Phys. Rep., 340, 291

Beers, T. C., Flynn, K., \& Gebhardt, K. 1990, AJ, 100, 32

Benítez, N. 2000, ApJ, 536, 571

Benítez, N., Ford, H., Bouwens, R., et al. 2004, ApJS, 150, 1

Bertin, E. 2006, in ASP Conf. Ser. 351, Astronomical Data Analysis Software and Systems XV, ed. C. Gabriel, C. Arviset, D. Ponz, \& S. Enrique (San Francisco, CA: ASP), 112

Bertin, E., \& Arnouts, S. 1996, A\&AS, 117, 393

Bhattacharya, S., Habib, S., \& Heitmann, K. 2011, arXiv:1112.5479

Biviano, A., \& Salucci, P. 2006, A\&A, 452, 75

Broadhurst, T. J., \& Barkana, R. 2008, MNRAS, 390, 1647

Broadhurst, T., Benítez, N., Coe, D., et al. 2005b, ApJ, 621, 53

Broadhurst, T., Takada, M., Umetsu, K., et al. 2005a, ApJ, 619, L143

Broadhurst, T. J., Taylor, A. N., \& Peacock, J. A. 1995, ApJ, 438, 49

Broadhurst, T., Umetsu, K., Medezinski, E., Oguri, M., \& Rephaeli, Y. 2008, ApJ, 685, L9

Bryan, G. L., \& Norman, M. L. 1998, ApJ, 495, 80

Bullock, J. S., Kolatt, T. S., Sigad, Y., et al. 2001, MNRAS, 321, 559

Cavagnolo, K. W., Donahue, M., Voit, G. M., \& Sun, M. 2009, ApJS, 182, 12

Clowe, D., Bradač, M., Gonzalez, A. H., et al. 2006, ApJ, 648, L109

Clowe, D., Luppino, G. A., Kaiser, N., \& Gioia, I. M. 2000, ApJ, 539, 540

Coe, D., Benítez, N., Broadhurst, T., \& Moustakas, L. A. 2010, ApJ, 723, 1678

Coe, D., Benítez, N., Sánchez, S. F., et al. 2006, AJ, 132, 926

Coe, D., Umetsu, K., Zitrin, A., et al. 2012, arXiv:1201.1616

Colberg, J. M., Krughoff, K. S., \& Connolly, A. J. 2005, MNRAS, 359, 272

Colberg, J. M., White, S. D. M., Yoshida, N., et al. 2000, MNRAS, 319, 209

Colless, M., Dalton, G., Maddox, S., et al. 2001, MNRAS, 328, 1039

Duffy, A. R., Schaye, J., Kay, S. T., \& Dalla Vecchia, C. 2008, MNRAS, 390, L64

Duffy, A. R., Schaye, J., Kay, S. T., et al. 2010, MNRAS, 405, 2161

Ebeling, H., Edge, A. C., \& Henry, J. P. 2001, ApJ, 553, 668

Ebeling, H., Ma, C. J., Kneib, J.-P., et al. 2009, MNRAS, 395, 1213

Fedeli, C. 2012, MNRAS, in press (arXiv:1111.5780)

Fioc, M., \& Rocca-Volmerange, B. 1997, A\&A, 326, 950

Ford, H. C., Clampin, M., Hartig, G. F., et al. 2003, Proc. SPIE, 4854, 81

Ford, J., Hildebrandt, H., Van Waerbeke, L., et al. 2011, arXiv:1111.3698

Gao, L., Navarro, J. F., Frenk, C. S., et al. 2012, arXiv:1201.1940

Gavazzi, R., Fort, B., Mellier, Y., Pelló, R., \& Dantel-Fort, M. 2003, A\&A, 403, 11

Geller, M. J., Diaferio, A., \& Kurtz, M. J. 2011, AJ, 142, 133

Gilmour, R., Best, P., \& Almaini, O. 2009, MNRAS, 392, 1509

Graham, A. W., Merritt, D., Moore, B., Diemand, J., \& Terzić, B. 2006, AJ, 132,2701

Grillo, C., Eichner, T., Seitz, S., et al. 2010, ApJ, 710, 372

Grogin, N. A., \& Narayan, R. 1996, ApJ, 464, 92 
Haig, D. J., Ade, P. A. R., Aguirre, J. E., et al. 2004, Proc. SPIE, 5498, 78 Halkola, A., Seitz, S., \& Pannella, M. 2006, MNRAS, 372, 1425 Hamana, T., Miyazaki, S., Shimasaku, K., et al. 2003, ApJ, 597, 98 Hennawi, J. F., Dalal, N., Bode, P., \& Ostriker, J. P. 2007, ApJ, 654, 714 Heymans, C., Van Waerbeke, L., Bacon, D., et al. 2006, MNRAS, 368, 1323 Hildebrandt, H., Muzzin, A., Erben, T., et al. 2011, ApJ, 733, L30 Hoekstra, H. 2003, MNRAS, 339, 1155

Hoekstra, H., Franx, M., \& Kuijken, K. 2000, ApJ, 532, 88

Hoekstra, H., Hartlap, J., Hilbert, S., \& van Uitert, E. 2011, MNRAS, 412, 2095 Host, O. 2012, MNRAS, 420, L18

Ilbert, O., Capak, P., Salvato, M., et al. 2009, ApJ, 690, 1236

Jarosik, N., Bennett, C. L., Dunkley, J., et al. 2011, ApJS, 192, 14

Jee, M. J., Rosati, P., Ford, H. C., et al. 2009, ApJ, 704, 672

Jing, Y. P., \& Suto, Y. 2000, ApJ, 529, L69

Johnston, D. E., Sheldon, E. S., Tasitsiomi, A., et al. 2007, ApJ, 656, 27

Jullo, E., Kneib, J.-P., Limousin, M., et al. 2007, New J. Phys., 9, 447

Jullo, E., Natarajan, P., Kneib, J.-P., et al. 2010, Science, 329, 924

Kaiser, N. 1995, ApJ, 439, L1

Kaiser, N., \& Squires, G. 1993, ApJ, 404, 441

Kaiser, N., Squires, G., \& Broadhurst, T. 1995, ApJ, 449, 460

Kassiola, A., \& Kovner, I. 1993, ApJ, 417, 450

Kawaharada, M., Okabe, N., Umetsu, K., et al. 2010, ApJ, 714, 423

Keeton, C. R. 2001, arXiv:astro-ph/0102341

Kimble, R. A., MacKenty, J. W., O'Connell, R. W., \& Townsend, J. A. 2008, Proc. SPIE, 7010, 70101E

Kitayama, T., \& Suto, Y. 1996, ApJ, 469, 480

Klypin, A. A., Trujillo-Gomez, S., \& Primack, J. 2011, ApJ, 740, 102

Kneib, J.-P., Ellis, R. S., Smail, I., Couch, W. J., \& Sharples, R. M. 1996, ApJ, 471,643

Kneib, J.-P., Hudelot, P., Ellis, R. S., et al. 2003, ApJ, 598, 804

Kneib, J.-P., \& Natarajan, P. 2011, A\&AR, 19, 47

Komatsu, E., Smith, K. M., Dunkley, J., et al. 2011, ApJS, 192, 18

Lau, E. T., Nagai, D., Kravtsov, A. V., \& Zentner, A. R. 2011, ApJ, 734, 93

Lemze, D., Barkana, R., Broadhurst, T. J., \& Rephaeli, Y. 2008, MNRAS, 386, 1092

Lemze, D., Broadhurst, T., Rephaeli, Y., Barkana, R., \& Umetsu, K. 2009, ApJ, 701,1336

Lemze, D., Wagner, R., Rephaeli, Y., et al. 2012, ApJ, 752, 141

Limousin, M., Richard, J., Jullo, E., et al. 2007, ApJ, 668, 643

Mahdavi, A., Hoekstra, H., Babul, A., Balam, D. D., \& Capak, P. L. 2007a, ApJ, 668,806

Mahdavi, A., Hoekstra, H., Babul, A., \& Henry, J. P. 2008, MNRAS, 384, 1567

Mahdavi, A., Hoekstra, H., Babul, A., et al. 2007b, ApJ, 664, 162

Mamon, G. A., Biviano, A., \& Murante, G. 2010, A\&A, 520, A30

Markevitch, M., Gonzalez, A. H., Clowe, D., et al. 2004, ApJ, 606, 819

Massey, R., Heymans, C., Bergé, J., et al. 2007, MNRAS, 376, 13

Mead, J. M. G., King, L. J., Sijacki, D., et al. 2010, MNRAS, 406, 434

Medezinski, E., Broadhurst, T., Umetsu, K., Benítez, N., \& Taylor, A. 2011, MNRAS, 414, 1840

Medezinski, E., Broadhurst, T., Umetsu, K., et al. 2007, ApJ, 663, 717

Medezinski, E., Broadhurst, T., Umetsu, K., et al. 2010, MNRAS, 405, 257

Meneghetti, M., Fedeli, C., Pace, F., Gottlöber, S., \& Yepes, G. 2010a, A\&A, 519, A90

Meneghetti, M., Fedeli, C., Zitrin, A., et al. 2011, A\&A, 530, A17

Meneghetti, M., Rasia, E., Merten, J., et al. 2010b, A\&A, 514, A93

Merten, J., Cacciato, M., Meneghetti, M., Mignone, C., \& Bartelmann, M. 2009, A\&A, 500, 681

Merten, J., Coe, D., Dupke, R., et al. 2011, MNRAS, 417, 333

Miyazaki, S., Komiyama, Y., Sekiguchi, M., et al. 2002, PASJ, 54, 833

Morandi, A., Limousin, M., Rephaeli, Y., et al. 2011, MNRAS, 416, 2567

Mroczkowski, T. 2011, ApJ, 728, L35

Nagai, D., Kravtsov, A. V., \& Vikhlinin, A. 2007, ApJ, 668, 1

Narikawa, T., \& Yamamoto, K. 2012, J. Cosmol. Astropart. Phys., JCAP05(2012)016

Navarro, J. F., Frenk, C. S., \& White, S. D. M. 1997, ApJ, 490, 493

Navarro, J. F., Ludlow, A., Springel, V., et al. 2010, MNRAS, 402, 21

Neto, A. F., Gao, L., Bett, P., et al. 2007, MNRAS, 381, 1450

Newman, A. B., Treu, T., Ellis, R. S., et al. 2009, ApJ, 706, 1078
Nonino, M., Dickinson, M., Rosati, P., et al. 2009, ApJS, 183, 244

Oguri, M., Bayliss, M. B., Dahle, H., et al. 2012, MNRAS, 420, 3213

Oguri, M., \& Blandford, R. D. 2009, MNRAS, 392, 930

Oguri, M., Hennawi, J. F., Gladders, M. D., et al. 2009, ApJ, 699, 1038

Oguri, M., \& Takada, M. 2011, Phys. Rev. D, 83, 023008

Oguri, M., Takada, M., Okabe, N., \& Smith, G. P. 2010, MNRAS, 405, 2215

Oguri, M., Takada, M., Umetsu, K., \& Broadhurst, T. 2005, ApJ, 632, 841

Okabe, N., Takada, M., Umetsu, K., Futamase, T., \& Smith, G. P. 2010, PASJ, 62,811

Okabe, N., \& Umetsu, K. 2008, PASJ, 60, 345

Okura, Y., \& Futamase, T. 2012, ApJ, 748, 112

Okura, Y., Umetsu, K., \& Futamase, T. 2008, ApJ, 680, 1

Peacock, J. A., \& Dodds, S. J. 1996, MNRAS, 280, L19

Percival, W. J., Reid, B. A., Eisenstein, D. J., et al. 2010, MNRAS, 401, 2148

Postman, M., Coe, D., Benítez, N., et al. 2012, ApJS, 199, 25

Prada, F., Klypin, A. A., Cuesta, A. J., Betancort-Rijo, J. E., \& Primack, J. 2011, arXiv: 1104.5130

Press, W. H., Teukolsky, S. A., Vetterling, W. T., \& Flannery, B. P. 1992, Numerical Recipes in FORTRAN. The Art of Scientific Computing (2nd ed.; Cambridge: Cambridge Univ. Press)

Rasia, E., Meneghetti, M., Martino, R., et al. 2012, New J. Phys., 14, 055018

Reese, E. D., Mroczkowski, T., Menanteau, F., et al. 2012, ApJ, 751, 12

Richard, J., Pei, L., Limousin, M., Jullo, E., \& Kneib, J. P. 2009, A\&A, 498, 37

Riess, A. G., Filippenko, A. V., Challis, P., et al. 1998, AJ, 116, 1009

Romanowsky, A. J., \& Kochanek, C. S. 1998, ApJ, 493, 641

Rozo, E., \& Schmidt, F. 2010, arXiv:1009.5735

Saha, P., \& Williams, L. L. R. 2004, AJ, 127, 2604

Sayers, J., Czakon, N. G., \& Golwala, S. R. 2012, ApJ, 744, 169

Sayers, J., Golwala, S. R., Ameglio, S., \& Pierpaoli, E. 2011, ApJ, 728, 39

Sereno, M., \& Umetsu, K. 2011, MNRAS, 416, 3187

Sereno, M., \& Zitrin, A. 2012, MNRAS, 419, 3280

Shaw, L. D., Weller, J., Ostriker, J. P., \& Bode, P. 2006, ApJ, 646, 815

Tasitsiomi, A., Kravtsov, A. V., Gottlöber, S., \& Klypin, A. A. 2004, ApJ, 607, 125

Tegmark, M., Blanton, M. R., Strauss, M. A., et al. 2004, ApJ, 606, 702

Umetsu, K. 2010, arXiv:1002.3952

Umetsu, K., Birkinshaw, M., Liu, G.-C., et al. 2009, ApJ, 694, 1643

Umetsu, K., \& Broadhurst, T. 2008, ApJ, 684, 177

Umetsu, K., Broadhurst, T., Zitrin, A., et al. 2011a, ApJ, 738, 41

Umetsu, K., Broadhurst, T., Zitrin, A., Medezinski, E., \& Hsu, L. 2011b, ApJ, 729,127

Umetsu, K., \& Futamase, T. 2000, ApJ, 539, L5

Umetsu, K., Medezinski, E., Broadhurst, T., et al. 2010, ApJ, 714, 1470

Umetsu, K., Takada, M., \& Broadhurst, T. 2007, Mod. Phys. Lett. A, 22, 2099

Van Waerbeke, L., Hildebrandt, H., Ford, J., \& Milkeraitis, M. 2010, ApJ, 723, L13

Vikhlinin, A., Kravtsov, A. V., Burenin, R. A., et al. 2009, ApJ, 692, 1060

Wechsler, R. H., Bullock, J. S., Primack, J. R., Kravtsov, A. V., \& Dekel, A. 2002, ApJ, 568, 52

Wojtak, R., Łokas, E. L., Mamon, G. A., et al. 2007, A\&A, 466, 437

Wright, C. O., \& Brainerd, T. G. 2000, ApJ, 534, 34

Wuyts, S., Labbé, I., Schreiber, N. M. F., et al. 2008, ApJ, 682, 985

Zhang, Y.-Y., Finoguenov, A., Böhringer, H., et al. 2008, A\&A, 482, 451

Zhang, Y.-Y., Reiprich, T. H., Finoguenov, A., Hudson, D. S., \& Sarazin, C. L. 2009, ApJ, 699, 1178

Zhao, D. H., Jing, Y. P., Mo, H. J., \& Börner, G. 2003, ApJ, 597, L9

Zhao, D. H., Jing, Y. P., Mo, H. J., \& Börner, G. 2009, ApJ, 707, 354

Zhao, H. 1996, MNRAS, 278, 488

Zheng, W., Postman, M., Zitrin, A., et al. 2012, arXiv:1204.2305

Zitrin, A., Broadhurst, T., Barkana, R., Rephaeli, Y., \& Benítez, N. 2011a, MNRAS, 410, 1939

Zitrin, A., Broadhurst, T., Coe, D., et al. 2011b, MNRAS, 413, 1753

Zitrin, A., Broadhurst, T., Coe, D., et al. 2011c, ApJ, 742, 117

Zitrin, A., Broadhurst, T., Umetsu, K., et al. 2009, MNRAS, 396, 1985

Zitrin, A., Broadhurst, T., Umetsu, K., et al. 2010, MNRAS, 408, 1916

Zitrin, A., Rosati, P., Nonino, M., et al. 2012, ApJ, 749, 97

Zwicky, F. 1959, Handbuch Phys., 53, 390 